Comprehensive Summaries of Uppsala Dissertations from the Faculty of Science and Technology 987

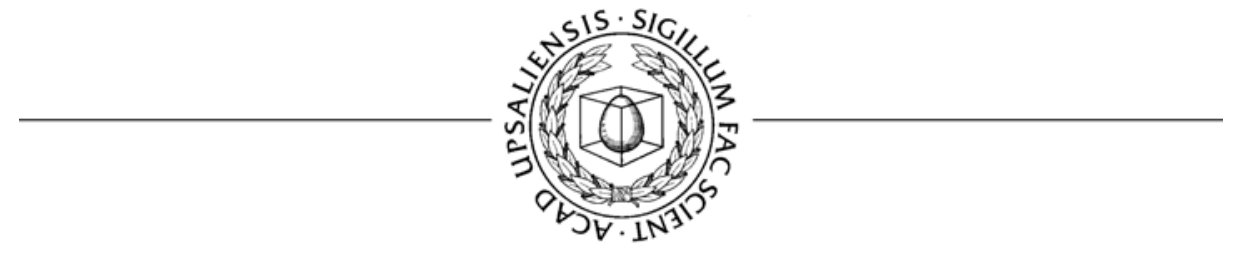

\title{
Resonant Soft X-Ray Emission Spectroscopy of Vanadium Oxides and Related Compounds
}

BY

THORSTEN SCHMITT

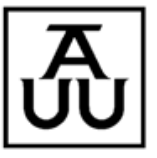

ACTA UNIVERSITATIS UPSALIENSIS UPPSALA 2004 
Dissertation presented at Uppsala University to be publicly examined in Polhemsalen, Ångström Laboratory, Uppsala, Monday, May 24, 2004 at 10:15 for the degree of Doctor of Philosophy. The examination will be conducted in English.

\begin{abstract}
Schmitt, T. 2004. Resonant Soft X-Ray Emission Spectroscopy of Vanadium Oxides and Related Compounds. (Resonant Mjukröntgenemissionsspektroskopi av Vanadinoxider och Relaterade Föreningar). Acta Universitatis Upsaliensis. Comprehensive Summaries of Uppsala Dissertations from the Faculty of Science and Technology 987.76 pp. Uppsala. ISBN 91-554-5987-0
\end{abstract}

This thesis addresses the electronic structure of vanadium and copper oxides using soft X-ray absorption (SXA) spectroscopy and resonant inelastic X-ray scattering (RIXS) at high brightness synchrotron radiation sources. In RIXS incident photons, tuned to the energy of specific absorption resonances, are inelastically scattered leaving behind a low energy valence excitation in the system studied. Effects of electron localization are reflected by the occurrence of low-energy excitations in form of dd- and charge-transfer excitations that are modelled by cluster calculations. Band-like states are dominating when the intermediate core excited state is delocalized.

RIXS at V 2p and O 1s resonances has been used to study the electronic structure of the monovalent vanadium oxides $\mathrm{VO}_{2}$ and $\mathrm{V}_{2} \mathrm{O}_{3}$, and of the mixed valence compounds, $\mathrm{NaV}_{2} \mathrm{O}_{5}$ and $\mathrm{V}_{6} \mathrm{O}_{13}$. For $\mathrm{NaV}_{2} \mathrm{O}_{5}$ and $\mathrm{V}_{6} \mathrm{O}_{13}$ significant contributions from localized low-energy excitations reflect the partly localized character of their valence band electronic structure, whereas $\mathrm{VO}_{2}$ and $\mathrm{V}_{2} \mathrm{O}_{3}$ appear mostly as band-like. Effects of carrier doping are addressed for the case of Mo doping into $\mathrm{VO}_{2}$ and reveal a quasi-rigid band behavior. In the cases of $\mathrm{VO}_{2}$ and $\mathrm{V}_{6} \mathrm{O}_{13}$ the temperature dependent metal-insulator transition could be monitored by following the spectral evolution of bands originating from V $3 d$ and V $3 d-O 2 p$ hybridized states. For $\mathrm{Na}_{2} \mathrm{~V}_{3} \mathrm{O}_{7}$ nanotubes it was possible to selectively probe states from the apical and the basal oxygen sites of $\mathrm{VO}_{5}$ pyramids that constitute these nanotubes. Furthermore, the RIXS technique has been demonstrated to be highly valuable in characterizing the charge transfer processes that accompany lithium insertion into vanadium oxide battery cathodes. Finally, for insulating cuprates RIXS at $\mathrm{O} 1 \mathrm{~s}, \mathrm{Cu} 3 \mathrm{p}$ and $\mathrm{Cu} 3 \mathrm{~s}$ resonances has been recorded at high-resolution for the detailed investigation of crystal field excitations.

Keywords: vanadium oxides, Li-battery, cathode materials, copper oxides, soft X-ray absorption, soft X-ray emission, resonant inelastic X-ray scattering, cluster model calculations

Thorsten Schmitt, Department of Physics, Box 530, Uppsala University, SE-75121 Uppsala, Sweden

(C) Thorsten Schmitt 2004

ISSN 1104-232X

ISBN 91-554-5987-0

urn:nbn:se:uu:diva-4290 (http://urn.kb.se/resolve?urn=urn:nbn:se:uu:diva-4290) 
To Florentina \& my parents 


\section{List of papers}

This thesis is based on a collection of articles listed below. Each paper will be referred to in the text by its Roman numeral.

I. Bandlike and excitonic states of oxygen in $\mathrm{CuGeO}_{3}$ : Observation using polarized resonant soft $\mathrm{X}$-ray emission spectroscopy

L.-C. Duda, J. Downes, C. McGuinness, T. Schmitt, A. Augustsson, K.E. Smith, G. Dhalenne, and A. Revcolevschi

Phys. Rev. B 61, 2000, 4186

II. Resonant Soft X-ray Emission Spectroscopy of $\mathrm{V}_{2} \mathrm{O}_{3}, \mathrm{VO}_{2}$ and $\mathrm{NaV}_{2} \mathrm{O}_{5}$

T. Schmitt, L.-C. Duda, A. Augustsson, J.-H. Guo, J. Nordgren, J.E. Downes, C. McGuinness, K.E. Smith, G. Dhalenne, A. Revcolevschi, M. Klemm, and S. Horn

Surf. Rev. Lett. 9, 2002, 1369

III. Resonant Inelastic Soft X-ray Scattering from Insulating Cuprates

L.-C. Duda, T. Schmitt, J. Nordgren, G. Dhalenne, and A. Revcolevschi Surf. Rev. Lett. 9, 2002, 1103

IV. The Redox Behavior of Vanadium Oxide Nanotubes As Studied by X-ray Photoelectron Spectroscopy and Soft X-ray Absorption Spectroscopy

S. Nordlinder, A. Augustsson, T. Schmitt, J.-H. Guo, L.-C. Duda, J. Nordgren, T. Gustafsson, and K. Edström

Chem. Mater. 15, 2003, 3227

V. The electronic structure and lithiation of electrodes based on vanadium-oxide nanotubes

A. Augustsson, T. Schmitt, L.-C. Duda, J. Nordgren, S. Nordlinder,

K. Edström, T. Gustafsson, and J.-H. Guo

J. Appl. Phys. 94, 2003, 5083

VI. Resonant soft X-ray emission spectroscopy of doped and undoped vanadium oxides

T. Schmitt, L.-C. Duda, M. Matsubara, A. Augustsson, F. Trif, J.-H. Guo,

L. Gridneva, T. Uozumi, A. Kotani, and J. Nordgren

J. Alloys. Comp. 362, 2004, 143

VII. Resonant soft X-ray emission of solids and liquids L.-C. Duda, T. Schmitt, A. Augustsson, and J. Nordgren J. Alloys. Comp. 362, 2004, 116 
VIII. Electronic structure studies of $\mathrm{V}_{6} \mathrm{O}_{13}$ by soft $\mathrm{X}$-ray emission spectroscopy: band-like and excitonic vanadium states T. Schmitt, L.-C. Duda, M. Matsubara, M. Mattesini, M. Klemm, A. Augustsson, J.-H. Guo, T. Uozumi, S. Horn, R. Ahuja, A. Kotani, and J. Nordgren Phys. Rev. B 69, 2004, 125103

IX. Li-insertion into $\mathrm{V}_{6} \mathrm{O}_{13}$ battery cathodes studied by soft X-ray spectroscopies

T. Schmitt, A. Augustsson, L.-C. Duda, J. Nordgren, J. Höwing, and T. Gustafsson Accepted for publication in J. Appl. Phys.

X. Electronic Structure of Li-inserted $\mathbf{V}_{6} \mathbf{O}_{13}$ Battery Cathodes: Rigid Band Behavior and Effects of Hybridization

T. Schmitt, A. Augustsson, J. Nordgren, L.-C. Duda, J. Höwing, T. Gustafsson, U. Schwingenschlögl, and V. Eyert

Submitted to Appl. Phys. Lett.

XI. Temperature dependent resonant soft X-ray emission study of the metal-semiconductor transition in $\mathrm{VO}_{2}$

T. Schmitt, L.-C. Duda, M. Mattesini, A. Augustsson, C. L. Dong, J.H. Guo, M. Klemm, V. Eyert, S. Horn, R. Ahuja, and J. Nordgren In manuscript

XII. Resonant inelastic soft X-ray scattering of $\mathrm{Na}_{2} \mathrm{~V}_{3} \mathrm{O}_{7}$ nanotubes T. Schmitt, L.-C. Duda, M. Matsubara, M. Mattesini, C. L. Dong, J.H. Guo, P. Millet, T. Uozumi, R. Ahuja, A. Kotani, and J. Nordgren In manuscript

XIII. Resonant Raman Scattering at the $\mathrm{Cu} 3 \mathrm{p}$ - resonances of $\mathrm{CuGeO}_{3}, \mathrm{SrCuO}_{2}, \mathrm{CuO}$, and $\mathrm{La}_{2} \mathrm{CuO}_{4}$

L.-C. Duda, T. Schmitt, J. Nordgren, P. Kuiper, C. de Graaf, J.-H. Guo, S. Canton, J. Bozek, G. Dhalenne, and A. Revcolevschi

In manuscript

XIV. Polarization dependent resonant inelastic soft X-ray scattering at the $\mathrm{O}$ 1s resonance of $\mathrm{SrCuO}_{2}$

T. Schmitt, L.-C. Duda, J. Nordgren, J.-H. Guo, G. Dhalenne, and A. Revcolevschi

In manuscript

XV. Comment: Low-energy excitations in resonant inelastic X-ray scattering of $\alpha^{\prime}-\mathrm{NaV}_{2} \mathrm{O}_{5}$

L.-C. Duda, T. Schmitt, J. Nordgren, P. Kuiper, G. Dhalenne, and A. Revcolevschi

Submitted to Phys. Rev. Lett.

Reprints were made with permission from the publishers. 
The following articles have been omitted from the thesis. They were omitted either due to the character of the material, or due to the limited extent of my contribution.

- Electronic Structure of GaAs $\mathbf{G}_{1-x} \mathbf{N}_{x}$ Alloy by Soft-X-Ray Absorption and Emission: Origin of the Reduced Optical Efficiency V.N. Strocov, P.O. Nilsson, A. Augustsson, T. Schmitt, D. DebowskaNilsson, R. Claessen, A. Yu. Egorov, V.M. Ustinov, Zh. I. Alferov Phys. Stat. Sol. (b) 233, 2002, R1

- Nitrogen local electronic structure in $\mathrm{Ga}(\mathrm{In}) \mathrm{AsN}$ alloys by soft Xray absorption and emission: Implications for optical properties V.N. Strocov, P.O. Nilsson, T. Schmitt, A. Augustsson, L. Gridneva, D. Debowska-Nilsson, R. Claessen, A. Yu. Egorov, V.M. Ustinov, and Zh. I. Alferov

Phys. Rev. B 69, 2004, 035206

- Local Electronic Structure of N Atoms in Ga(In)AsN by Soft Xray Absorption and Emission: Optical Efficiency

V.N. Strocov, P.O. Nilsson, T. Schmitt, R. Claessen, A. Yu. Egorov, V.M. Ustinov, and Zh. I. Alferov

Accepted for publication in Int. J. Nanoscience.

- Band structure effects in nitrogen $\mathrm{K}$-edge resonant inelastic $\mathrm{X}$ ray scattering from GaN

V.N. Strocov, T. Schmitt, J.-E. Rubensson, P. Blaha, T. Paskova, and P.O. Nilsson

Phys. Stat. Sol. (b) 241, 2004, R27

- New type of RIXS processes in semiconductor crystals M. Adell, J. Kanski, L. Ilver, and T. Schmitt

In manuscript

- Electronic structure of nanostructured $\mathrm{ZnO}$ from X-ray absorption and emission spectroscopy and LDA calculation

C. L. Dong, C. Persson, L. Vayssieres, A. Augustsson, T. Schmitt, M. Mattesini, R. Ahuja, C. L. Chang, and J.-H. Guo

Submitted to Phys. Rev. B

- Study of the defects in $\mathrm{ZnO}$ nanorods by means of soft-x-ray absorption and emission spectroscopy

C. L. Dong, C. Persson, A. Augustsson, T. Schmitt, M. Mattesini, L. Vayssieres, S. Mao, R. Ahuja, C. L. Chang, and J.-H. Guo

In manuscript

- Chemical modification in wear tracks of CVD diamond surfaces studied with X-ray absorption spectroscopy

L.-C. Duda, T. Schmitt, J. Nordgren, J. Andersson, and S. Jacobson

Submitted to Appl. Phys. Lett. 
- 3d metal $\mathbf{L}_{\alpha, \beta}$ resonant $\mathbf{X}$-ray emission and $\mathbf{L}_{2,3}$ absorption of Heusler alloys

M.V. Yablonskikh, Yu.M. Yarmoshenko, I.V. Soloview, E.Z. Kurmaev, L.C. Duda, T. Schmitt, M. Magnusson, J. Nordgren, and A. Moewes In manuscript

- Resonant Inelastic Soft X-Ray Scattering at Hollow Lithium States in Solid $\mathrm{LiCl}$

M. Agåker, J. Söderström, T. Käämbre, C. Glover, L. Gridneva, T. Schmitt, A. Augustsson, M. Mattesini, R. Ahuja, and J.-E. Rubensson Submitted to Phys. Rev. Lett.

- Low energy excitations in $\mathrm{Li}_{2} \mathrm{CuO}_{2}$ observed by $\mathrm{O} \mathrm{K}$ RIXS

T. Learmonth, C. McGuinness, J. Downes, P.-A. Glans, T. Schmitt, Y. Zhang, L.-C. Duda, J.-H. Guo, K. Smith, and F. Chou

In manuscript

- Resonant soft x-ray emission spectroscopy of $\mathrm{Na}_{0.7} \mathrm{CoO}_{2}$ and $\mathrm{Na}_{x} \mathrm{CoO}_{2} \bullet \mathrm{y} \mathrm{H}_{2} \mathrm{O}$

L.-C. Duda, T. Schmitt, J. Nordgren, J.-H. Guo, A. Pohl, G. Westin, and P. Svedlindh

In manuscript

- Diamond: A high-friction material in disguise J. Andersson, L.-C. Duda, T. Schmitt, and S. Jacobson In manuscript

\section{Comments on my participation}

Experimental studies performed at synchrotron facilities, which is the case for most of the work presented here, are always an effort of many people, which is also reflected by the lengthy author lists. My contribution to the papers has been on the experimental side, as well as in the analysis, discussions and writing of the papers. I have taken part in all the experiments, and in the preparations of most of them. I am responsible for the data analysis and writing of Papers II, VI, VIII, IX, X, XI, XII, and XIV. My contribution to the discussion and writing of the other papers is to some extent described by the position in the author list. 



\section{Contents}

1 Introduction 11

2 Theoretical background of soft X-ray spectroscopies 13

2.1 General background of soft X-ray spectroscopies . . . . . . . . . 13

2.2 Quantum theory of radiation . . . . . . . . . . . . 17

2.3 X-ray absorption spectroscopy . . . . . . . . . . . . . 21

3 Theoretical Models 23

3.1 Crystal-field theory . . . . . . . . . . . . . . . 23

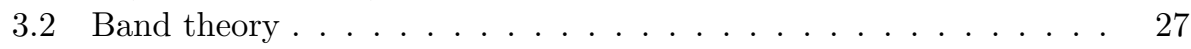

3.3 Hubbard Models . . . . . . . . . . . . . . . . . . . . . . . 28

3.4 Single impurity Anderson model . . . . . . . . . . . . . . 28

4 Experimental description 33

4.1 Synchrotron radiation sources _. . . . . . . . . . . . . 33

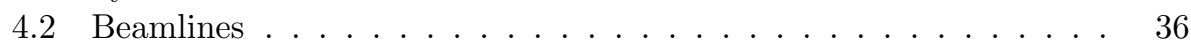

4.2 .1 Beamline 7.0 .1 at ALS . . . . . . . . . . . . . . . 37

4.2.2 Beamline I511-3 at MAX-Lab . . . . . . . . . . . . 38

4.3 Soft X-ray emission spectrometer . . . . . . . . . . . . . . . . 38

4.4 Sample preparations . . . . . . . . . . . . . . . . . . 42

4.4.1 Single crystals of vanadium and copper oxides . . . . . . . . 42

4.4 .2 Vanadium oxide battery cathodes . . . . . . . . . . . 42

5 Results 45

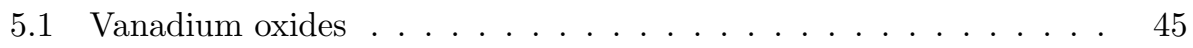

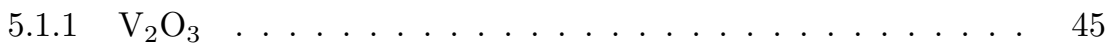

$5.1 .2 \quad \mathrm{VO}_{2}$ and $\mathrm{Mo}_{x} \mathrm{~V}_{1-x} \mathrm{O}_{2} \ldots \ldots \ldots \ldots \ldots$

5.1 .3 MIT in $\mathrm{VO}_{2} \ldots \ldots \ldots \ldots \ldots$

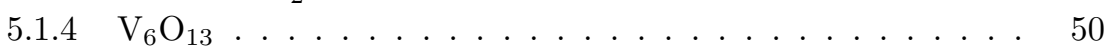

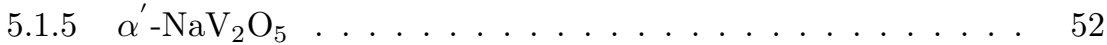

$5.1 .6 \quad \mathrm{Na}_{2} \mathrm{~V}_{3} \mathrm{O}_{7}$ nanotubes . . . . . . . . . . . . . 54

5.2 Lithium insertion into vanadium oxide cathodes . . . . . . . . . 56

$5.2 .1 \quad \mathrm{~V}_{6} \mathrm{O}_{13}$ battery cathodes $\ldots \ldots \ldots \ldots \ldots \ldots$

5.2 .2 Cathodes of vanadium oxide nanotubes . . . . . . . 60

5.3 Insulating Cuprates . . . . . . . . . . . . . . . . . 61 
5.3.1 Resonant SXE spectroscopy at O 1s resonances . . . . . . . 61

5.3.2 Resonant inelastic soft X-ray scattering at $\mathrm{Cu} 3 \mathrm{p}$ and $\mathrm{Cu} 3 \mathrm{~s}$ resonances . . . . . . . . . . . . . . 65

$\begin{array}{ll}\text { Acknowledgments } & 67\end{array}$

$\begin{array}{ll}\text { Summary in Swedish } & 69\end{array}$

$\begin{array}{ll}\text { Bibliography } & 72\end{array}$ 


\section{Chapter 1}

\section{Introduction}

Vanadium oxides belong to the important class of early $3 \mathrm{~d}$ transition metal compounds [1], which have received strong attention in research over the last decades. Owing to their broad variety of electronic, magnetic and structural properties and because of their interesting technological applications, they have been widely studied. Prominent examples of industrial applications range from the area of catalysis to optoelectronics, where vanadium oxides are for instance used for the construction of electrical switching devices [2] and smart thermochromic windows.

Chemical substitution facilitates to synthesize tailor made materials with fine tuned physical properties. Their relatively open crystal structure, consisting of distorted $\mathrm{VO}_{6}$ octahedra, makes it possible to introduce an alkali metal as a further component. Especially, $\mathrm{Li}^{+}$ions can achieve high mobilities in crystal lattices due to their extraordinary small radius. Therefore vanadium oxides have recently been studied also as promising cathode materials for rechargeable lithium batteries, where $\mathrm{Li}$ is inserted into vanadium oxide based materials [3,4].

A most important characteristic is, that vanadium oxides display metal-toinsulator or metal-to-semiconductor transitions $[5,6]$ as a function of the temperature, which are closely linked to potential value in their respective applications. Understanding the dual nature of their electronic structure, being governed by interplay of localized strongly correlated as well as delocalized (intinerant) $3 \mathrm{~d}$ electrons, is a formidable challenge for basic condensed matter and materials research.

Late $3 \mathrm{~d}$ transition metal oxides and in particular copper oxides, on the other hand are known to be strongly correlated systems, where the on-site d-d Coulomb repulsion at $\mathrm{Cu}$ sites, $\mathrm{U}_{d d}$, is larger than the d-band width $\mathrm{W}$ [7]. After the discovery of the high- $T_{c}$ superconducting cuprates also insulating cuprates have gained renewed interest $[1,7]$. Upon doping insulating copper oxides, for instance with Group IIb elements, transitions to the superconducting phase can be induced. Moreover, they can serve as ideal model systems for the understanding of the spectroscopic properties of the $\mathrm{Cu}-\mathrm{O}$ octahedra, the basic building blocks of the crystal structure of most copper oxides. Spectroscopic studies investigating the role of correlation effects for the electronic structure are of immense importance 
for the understanding of the basic origin of macroscopic transport properties.

The current studies are focused on the characterization of the electronic structure of vanadium and copper oxides by means of resonant soft X-ray emission (SXE) [8] and soft X-ray absorption (SXA) spectroscopy [9]. SXA and SXE spectroscopy probe unoccupied and occupied valence band states, respectively. In resonant SXE (RSXE) spectroscopy experiments monochromatized synchrotron radiation, tuned to the energy of specific resonances in the SXA spectrum of the material studied, is employed to selectively excite SXE [8].

Thereby atomic and even orbital sensitive information can be gained from the RSXE spectra, which characterizes the chemical state of specific atoms in the material. For many delocalized materials, like for instance wide band materials, the photon excitation and emission process can be viewed as two consecutive decoupled steps [10]. However, it is not always justified to consider the whole RSXE process as two separated processes. Moreover, for correlated materials the intermediate state after the photon excitation is rather localized and in order to accurately describe the electronic structure of such materials one needs to treat RSXE spectroscopy as a one step process described as resonant inelastic X-ray scattering (RIXS) $[8,11,12]$.

In this view the incident photon is inelastically scattered and thereby occurring spectral contributions due to (localized) low energy charge neutral excitations, like crystal field excitations (e.g. dd-excitations) or charge transfer (CT) excitations between delocalized ligand and localized transition metal orbitals, can be analyzed and explained. Such charge neutral excitations are particular prominent in correlated 3d transition metal oxides like copper and vanadium oxides, and their study allows to probe the degree of localization and the role of electron correlation effects.

The thesis is organized in the following way. In chapter $2 \mathrm{I}$ will describe the theoretical background relevant for the understanding of the performed soft X-ray spectroscopy investigations. Chapter 3 deals with theoretical models which are used to interpret the RSXE spectra of the studied solid vanadium and copper oxide samples. Chapter 4 gives a description of the experimental set-up used and describes the sample preparation. In chapter 5 all results of the included papers are summarized in great detail and ordered in separate sections dealing with vanadium oxide materials (paper II, VI, VIII, XI, XII and XV), vanadium oxide based Li-battery systems (paper IV, V, IX and X) and insulating cuprates (paper I, III, VII, XIII and XIV). 


\section{Chapter 2}

\section{Theoretical background of soft X-ray spectroscopies}

\subsection{General background of soft X-ray spectroscop- ies}

Spectroscopic techniques use interactions between photons and matter to probe the electronic structure and measure thereby the energy distribution of electronic states in atoms, molecules or solid state materials. By determining transition probabilities between these states it is possible to investigate the origin of chemical and physical material properties. Consequently, spectroscopy is nowadays one of the most powerful tools in modern physics. In particular, fundamental spectroscopic observations triggered the development of quantum mechanics. One prominent example for this is the measurement of the hydrogen spectrum by Anders Jonas Ångström in Uppsala, which was later described by the famous Balmer series.

The valence levels of atomic systems host the least bound electrons and the core levels have much higher binding energies. Therefore valence electrons are responsible for the bonding between atoms in molecules or solids, and the energy distribution of their states in occupied and unoccupied parts is related to chemical properties of the materials. Core states are spatially localized at a specific atom, and therefore core states of different elements are energetically well separated. Since electronic transitions between shallow core and valence levels correspond to photon energies that fall into the range of soft X-rays (ca. $20 \mathrm{eV}$ to $5 \mathrm{keV}$ ), radiation from this spectral region is extremely useful for the studies of electronic properties of various materials. Fig. $2.1-2.3$ depict different spectroscopic modes utilizing photons from the soft X-ray range.

Soft X-ray spectroscopies following from single photon excitation and ionization processes are summarized in Fig. 2.1. In soft X-ray absorption (SXA) spectroscopy (Fig. 2.1 (a)) [9,13], a core electron is promoted to an empty valence state by an incident X-ray photon of energy $\hbar \omega_{1}$. By varying $\hbar \omega_{1}$ in SXA spectroscopy one can map the unoccupied valence states. The atomic-like nature of the core hole 


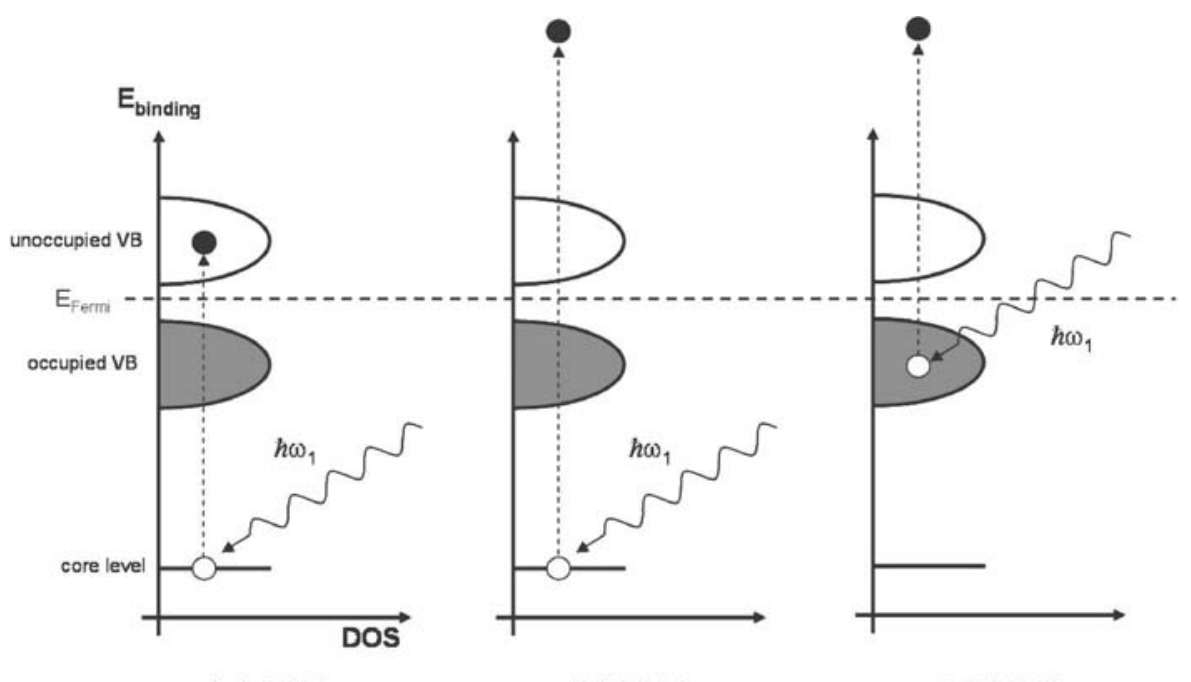

(a) SXA

(b) XPS

(c) UPS

Figure 2.1: Schematic illustrations of soft X-ray spectroscopies with one-photon processes in an insulator. Horizontal and vertical axes represent binding energy $\left(E_{\text {binding }}\right)$ and density of states $(D O S)$, respectively. The valence band (VB) is split into an upper unoccupied VB and a lower occupied VB with the Fermi level, $E_{F e r m i}$, between them. Note that the energy difference of the VB and the horizontal bar symbolizing the core level is underestimated. (a) Soft X-ray absorption (SXA), (b) X-ray Photoelectron Spectroscopy (XPS), and (c) Ultraviolet Photoelectron Spectroscopy (UPS).

makes SXA an elemental and site sensitive spectroscopic technique. Photoemission spectroscopy (PES) [14] measures the kinetic energy distribution of emitted photoelectrons that are excited to the high-energy continuum. Depending on the energy of the incident photons, PES is generally divided into X-ray Photoelectron spectroscopy (XPS) (see Fig. 2.1 (b)) or Ultraviolet photoelectron spectroscopy (UPS) (see Fig. 2.1 (c)), where XPS and UPS ionize core and valence states, respectively. The measured kinetic energy $E_{\text {kinetic }}$ of the emitted electrons can be related to the binding energy $E_{\text {binding }}$ by

$$
E_{\text {binding }}=\hbar \omega-E_{\text {kinetic }}-\phi \text {, }
$$

where $\hbar \omega$ is the energy of the incident photon and $\phi$ is the work function. Using UPS, which is sometimes also called valence band PES (VB-PES), one gets thereby information about the distribution of the occupied valence states, i.e. chemical information. XPS as a core level spectroscopy reveals direct elemental information and in addition also chemical information by the energy position and shape of the lines, which are dependent on the chemical environment. Therefore XPS is often also denoted as Electron Spectroscopy for Chemical Analysis (ESCA) [15]. SXA, XPS and UPS are first order optical processes in the sense that first order perturbation theory of the electron-photon interaction can be used to describe 
their properties.

After a core hole is created, the resulting core ionized (Fig. $2.2(1)$ ) or core excited (Fig. 2.3(I)) state will rapidly decay on a femtosecond time scale. In this decay process the vacant core level is filled by a valence electron in an electron transition, either by a non-radiative Auger decay involving the emission of an Auger electron (Fig. $2.2(2 \mathrm{~A})$ ) or by the fluorescence of an X-ray photon (Fig. 2.2 $(2 \mathrm{~B}))$.

The Auger electron yield for light elements dominates over the fluorescence yield in the soft X-ray region by a factor of 100-1000 [16,17]. This poses due to the relatively small solid angles of photon collection high demands on the required excitation intensities for soft X-ray fluorescence spectroscopy, which is nowadays mostly denoted as soft X-ray emission (SXE) spectroscopy [8]. Therefore SXE spectroscopy, which measures intensity and photon energy distribution of x-rays emitted from a sample, was long limited by the use of excitation sources delivering high-energetic electron beams of several keV kinetic energy, a technique which creates a core ionized state. The arrival of dedicated synchrotron radiation sources made it possible to employ monochromatized soft X-ray photons as excitation agents for SXE spectroscopy.

When the initial photon excitation process by incident soft X-ray photons of energies well above the ionization threshold results in a core ionized intermediate state, the following SXE process is generally denoted as normal SXE (NSXE) (see Fig. $2.2(1 / 2 \mathrm{~B}))[8,11]$. The intermediate state in NSXE is the same as the final state for XPS. Therefore, the SXE process in NSXE can be described independently from the excitation process, i.e., the excitation and subsequent radiative decay can be viewed as a two-step process. In this case NSXE can be described as spontaneous emission of X-ray photons in a dipole transition between two electronic states. NSXE is largely independent of the incident photon energy $\hbar \omega_{1}$ and thus often reflects partial density of states (PDOS) of the occupied valence band, if atomic multiplet effects can be neglected.

Furthermore, monochromatized photon excitation below the ionization threshold (see Fig. 2.3 (I)) enables to perform resonant soft X-ray emission (RSXE) spectroscopy [11]. RSXE is a second order optical process, where an incident photon of energy $\hbar \omega_{1}$ promotes a core electron to an empty valence level (Fig. 2.3 (I)) and subsequently a valence electron fills the core hole in a radiative transition emitting an X-ray photon of energy $\hbar \omega_{2}$ (Fig. 2.3 (IIb)). The final state of SXA spectroscopy is the intermediate state in RSXE. In contrast to XPS, which leaves the system in a ionized state, RSXE is a charge neutral process, i.e. the number of electrons in ground and final state is identical for a specific atomic site. If the core hole is filled by the same valence electron which was excited from the ground state, one speaks of direct re-emission or of an elastic contribution in RSXE (Fig. 2.3 (IIa)).

SXE spectroscopy has several advantages over other spectroscopies. It is as a photon-in/photon-out technique bulk sensitive, owing to the attenuation length of soft X-ray photons (ca. 100-200 nm), revealing complementary information to the surface sensitive electron spectroscopies. In addition to the common elemental selectivity of core level spectroscopies, RSXE greatly profits from the use 


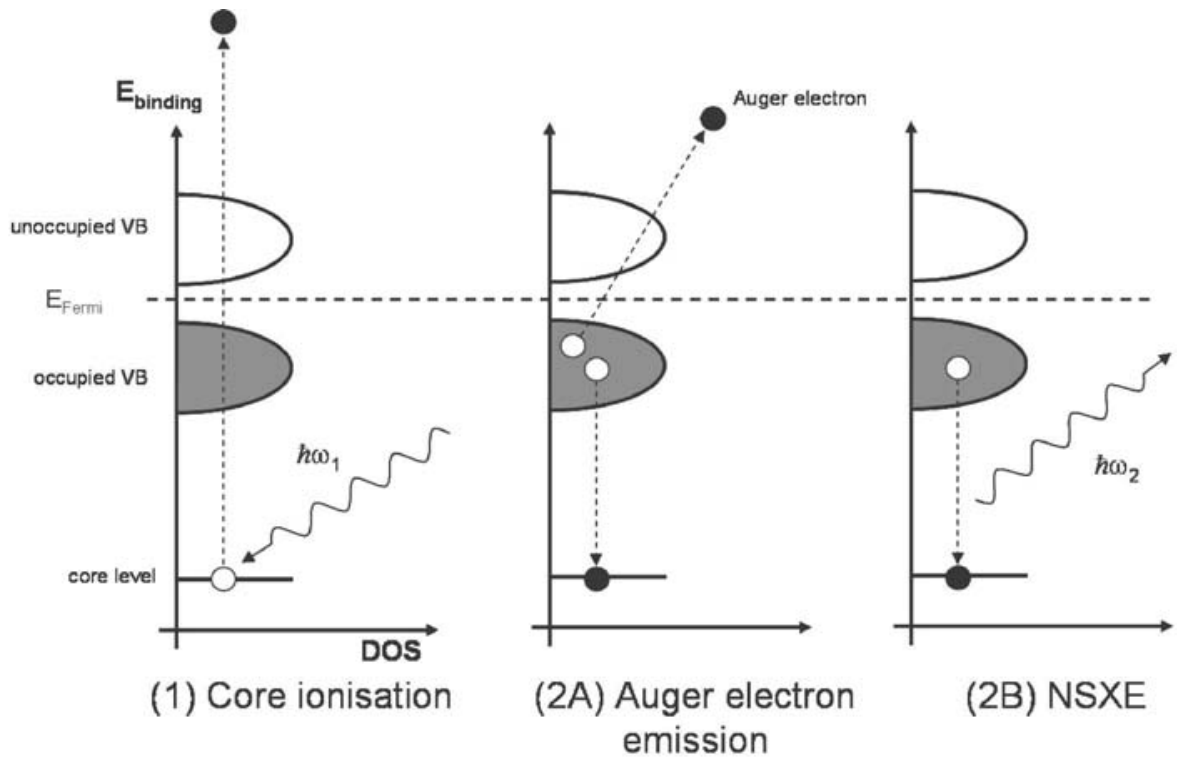

Figure 2.2: Schematic illustrations of the decay processes after an initial core ionization (1). (2A) Auger electron emission. (2B) Soft X-ray fluorescence or normal soft X-ray emission (NSXE)leaving the system in an valence ionized final state.

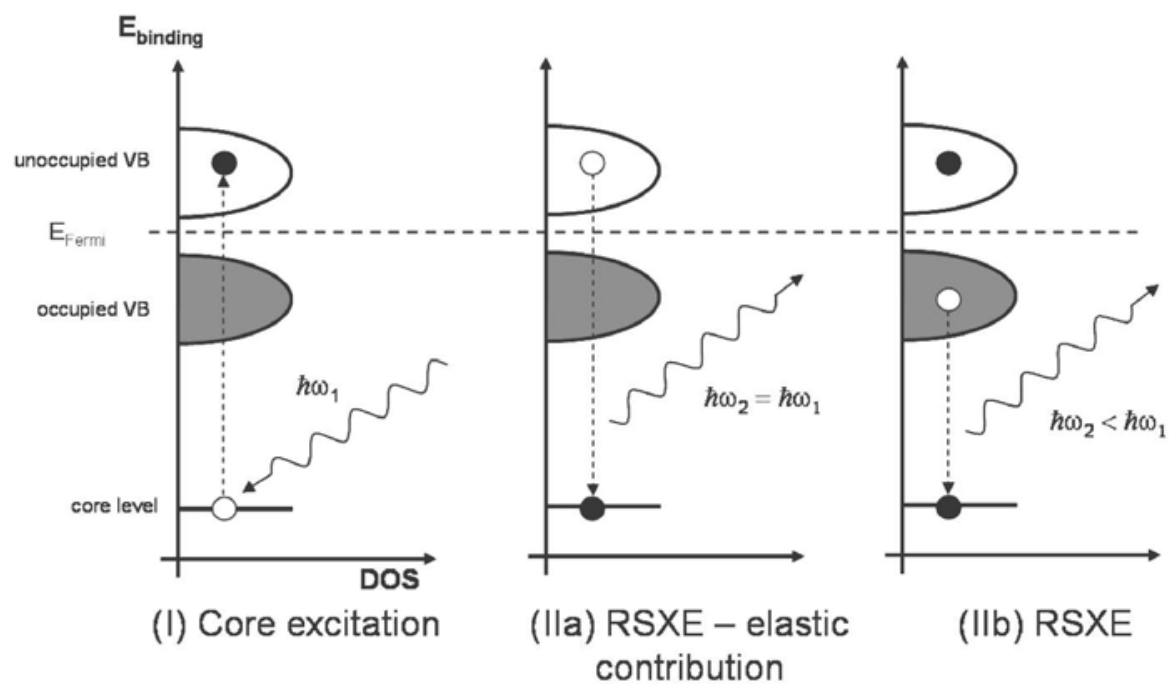

Figure 2.3: Schematic illustrations describing the processes of resonant soft X-ray emission (RSXE). (I) Core excitation. (IIa) elastic part of RSXE where the initial and final state are the same. (IIb) inelastic part of RSXE where the final state is a valence excited state. 
of selective excitation with synchrotron radiation in obtaining atomic site- and orbital-sensitive properties. This entails that RSXE reflects the PDOS projected onto specific atomic sites or, owing to the use of polarized X-rays and the virtue of dipole selection rules, onto orbitals of defined symmetry.

Tuning of monochromatized synchrotron radiation to photon excitation energies close to the core threshold RSXE reveals interesting additional resonance phenomena, which can not be explained by a two-step description. It was shown that RSXE must be treated as a coherent absorption-emission event, i.e. it must be regarded as an one-step process [18]. In this description one is dealing with a photon-in/photon-out scattering process and RSXE is then often denoted as resonant inelastic X-ray scattering (RIXS), which is described by inelastic scattering theory $[11,18]$. This entails that RSXE/RIXS can give valuable information on dynamic properties and, e.g., the influence of correlation effects in the electronic structure. After giving an descriptive overview about different soft X-ray spectroscopies and the underlying electron-photon processes, the next section will be devoted to the quantum mechanical description of SXA, NSXE and RIXS.

\subsection{Quantum theory of radiation}

\section{Interaction of light with matter}

In non-relativistic quantum mechanics [19] the electromagnetic radiation field $\mathbf{A}$ is a vector potential operator at position $\mathbf{r}_{i}$ and time $t$,

$$
\mathbf{A}=\mathbf{A}\left(\mathbf{r}_{i}, t\right)=\frac{c}{\sqrt{V}} \sum_{k} \sum_{\alpha} \sqrt{\frac{\hbar}{2 \omega}}\left[\mathbf{a}_{\mathbf{k}, \alpha} \hat{\epsilon}_{\alpha} e^{i \mathbf{k} \cdot \mathbf{r}_{i}-i \omega t}+\mathbf{a}_{\mathbf{k}, \alpha}^{\dagger} \hat{\epsilon}_{\alpha} e^{-i \mathbf{k} \cdot \mathbf{r}_{i}+i \omega t}\right]
$$

where $\mathbf{a}_{\mathbf{k}, \alpha}$ and $\mathbf{a}_{\mathbf{k}, \alpha}^{\dagger}$ are annihilation and creation operators, which decrease and increase the number of photons by one, respectively. In Eq. (2.2) $\mathbf{k}$ and $\alpha$ stand for wave vectors and spin quantum numbers of the material system. $\hat{\epsilon}_{\alpha}$ are unitary polarization vectors and $\omega$ gives the photon frequency. For the electrons $i$ of an atom in a quantum mechanical system describing some material the interaction Hamiltonian can be written as:

$$
H_{i n t}=\frac{e}{2 m c} \sum_{i}\left(-2 \mathbf{A} \cdot \mathbf{p}_{i}+\frac{e}{c} \mathbf{A} \cdot \mathbf{A}\right) .
$$

Eq. (2.3) gives together with the Hamiltonian of the unperturbed system,

$$
H_{0}=\sum_{i}\left(\frac{1}{2 m} \mathbf{p}_{i}+V\left(\mathbf{r}_{i}\right)\right)
$$

for the total Hamiltonian of the system:

$$
H=H_{0}+H_{i n t}=\sum_{i}\left(\frac{1}{2 m}\left(\mathbf{p}_{i}+e \mathbf{A}\right)^{2}+V\left(\mathbf{r}_{i}\right)\right),
$$


where $\mathbf{p}_{i}$ is the momentum vector of electron i and $V\left(\mathbf{r}_{i}\right)$ is the scalar potential at electron $i$ of the system under consideration.

\section{Fermi's Golden Rule}

The vector potential $\mathbf{A}\left(\mathbf{r}_{i}, t\right)$ is a quantized field operator and is written in Eq. (2.2) as a superposition of creation and annihilation operators, which are directly related to the photon number operator. For a single electron transition, i.e. a single photon emission or absorption event, the eigenvalue of the photon number operator has to change by exactly one. The second term $\propto \mathbf{A} \cdot \mathbf{A}$ in Eq. (2.3) for the interaction Hamiltonian changes the total number of photons by 0 or \pm 2 and has in the lowest order of perturbation theory no contribution to one photon processes. For single photon processes only the left term of Eq. (2.3) contributes and the transition probability is proportional to the squared matrix element of the interaction Hamiltonian Eq. (2.3) between the initial $|i\rangle$ and final $|f\rangle$ photon state. It is straightforward to derive the transition probability $w_{i, f}$ per unit time from the total Hamiltonian in Eq. (2.5) employing time dependent perturbation theory treating $H_{i n t}$ as a small perturbation. The famous result is Fermi's Golden Rule,

$$
w_{i, f}=\frac{2 \pi}{\hbar}\left|\left\langle f\left|H_{i n t}\right| i\right\rangle\right|^{2} \delta\left(E_{f}-E_{i} \mp \hbar \omega\right),
$$

where the delta distribution takes care of energy conservation, $E_{f}-E_{i} \mp \hbar \omega=0$, between final and initial states, and $-\hbar \omega$ or $+\hbar \omega$ refer to the absorption or emission of a photon, respectively.

\section{Transition rate of the X-ray emission process}

In the soft X-ray region photon wavelengths are much larger than atomic dimensions or crystal lattice parameters. Therefore the the scalar product $\mathbf{k} \cdot \mathbf{r}_{i}$ must be much less than unity and in a series expansion of Eq. (2.2) we can approximate for the exponential term $e^{\left(i \mathbf{k} \cdot \mathbf{r}_{\mathbf{i}}\right)} \approx 1$. Assuming emission of a photon in a continuous energy interval $[\hbar \omega, \hbar(\omega+d \omega)]$ into a solid angle element $d \Omega$ the number of allowed states is $\rho d(\hbar \omega) \propto \omega^{2} d \Omega d(\hbar \omega)$. Multiplying by the latter, integrating Eq. (2.6) over the photon energies $d(\hbar \omega)$ by using Eq. (2.2) and Eq. (2.3) together with the approximations above leads to the transition rate:

$$
w_{d \Omega} \propto \omega\left|\left\langle f\left|\hat{\epsilon}_{\alpha} \cdot \mathbf{p}\right| i\right\rangle\right|^{2} d \Omega .
$$

Rewriting Eq. (2.7) with the use of the commutation relation between position operator $\mathbf{r}$ and the unperturbed Hamiltonian $\left(\mathbf{p}=\frac{\mu}{i \hbar}\left[\mathbf{r}, H_{0}\right]\right)$ gives the familiar form [19],

$$
w_{d \Omega} \propto \omega^{3}\left|\left\langle f\left|\hat{\epsilon}_{\alpha} \cdot \mathbf{r}\right| i\right\rangle\right|^{2} d \Omega,
$$

with the electric dipole operator $\left(\hat{\epsilon}_{\alpha} \cdot \mathbf{r}\right)$. Eq. (2.8) for the transition rate of emitted photons into the solid angle element $d \Omega$ is written in the electric dipole 
approximation, which approximates the vector field by an oscillating dipole. In Eq. (2.7) and (2.8) is implicitly assumed that only one electron participates in the spontaneous emission process, i.e. NSXE or ordinary fluorescence is described.

\section{Electric dipole selection rules}

Angular integration of Eq. (2.8) imposes dipole selection rules for single photon transitions by determining the non-zero matrix elements. They state that only transitions are allowed, which change the angular momentum quantum number $l$ by one, $\Delta l= \pm 1$. In addition the z-component of the orbital momentum quantum number $m$ has to fulfill $\Delta m= \pm 1,0$ and the spin $s$ must be conserved, i.e. $\Delta s=0$. Note that the dipole selection rules are not just restricted to systems consisting of single atoms. Furthermore, it is generally possible to expand the system's wave functions by atomic-like wave functions when dealing with molecules and solid materials and thus the dipole selection rules above are still valid (but sometimes less stringent as for atoms) for all kinds of electronic systems.

The formulas above were derived for single photon processes like the absorption or emission of an X-ray photon. For the case of X-ray emission this approach is only justified for describing a spontaneous transition from an core ionized state to a valence excited state, which is generally referred to as non-resonant X-ray emission or normal soft X-ray emission (NSXE). In practice there are two common experimental excitation techniques which result in core ionization of a system, either by electrons or X-ray photons, both with energies exceeding the ionization limit. However, if the excitation occurs with X-ray photons tuned to photon energies close to an absorption resonance of the material, it is no longer possible to separate the excitation and emission process into two consecutive steps. Instead, one has to deal with a one-step process described by inelastic scattering theory $[18,19]$.

\section{Resonant inelastic X-ray scattering}

In order to evaluate the transition probability for an one-step scattering process the interaction Hamiltonian from Eq. (2.3) is used. An one-step scattering process will not change the total number of photons and, therefore the left term in Eq. (2.3) $\propto \mathbf{A} \cdot \mathbf{p}_{i}$ will not contribute to it in first order of perturbation theory. However, the second order of the perturbation theory gives a contribution from this term. The second term of Eq. (2.3), which is $\propto \mathbf{A} \cdot \mathbf{A}$, contributes as pointed out above to transitions without change in the number of photons. The differential cross section for inelastic X-ray scattering is given by the Kramers-Heisenberg formula [18-21]

$$
\begin{array}{r}
\frac{d \sigma}{d \omega_{2} d \Omega} \propto \frac{\omega_{2}}{\omega_{1}} \sum_{f} \mid \frac{e^{2}}{2 m c^{2}}\left\langle f\left|\mathbf{A}_{2} \cdot \mathbf{A}_{1}\right| i\right\rangle+\frac{e}{m c} \sum_{m}\left[\frac{\left\langle f\left|\mathbf{p}_{2} \cdot \mathbf{A}_{2}\right| m\right\rangle\left\langle m\left|\mathbf{p}_{1} \cdot \mathbf{A}_{1}\right| i\right\rangle}{E_{m}-E_{i}-\hbar \omega_{1}-\frac{i \Gamma_{m}}{2}}\right. \\
\left.+\frac{\left\langle f\left|\mathbf{p}_{2} \cdot \mathbf{A}_{2}\right| m\right\rangle\left\langle m\left|\mathbf{p}_{1} \cdot \mathbf{A}_{1}\right| i\right\rangle}{E_{i}-E_{m}+\hbar \omega_{2}}\right]\left.\right|^{2} \times \delta\left(\hbar \omega_{1}-\hbar \omega_{2}-E_{f}+E_{i}\right),
\end{array}
$$




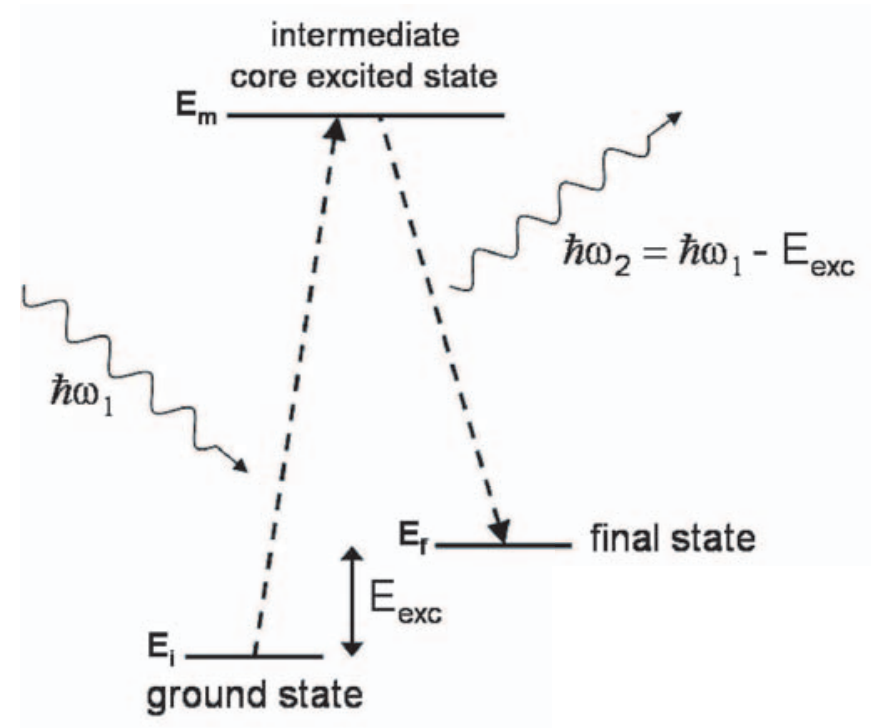

Figure 2.4: Schematic energy level diagram of the one-step process in resonant inelastic X-ray scattering (RIXS) in a atomic-like system. $\mathrm{E}_{\text {exc }}$ represents the energy of a localized low-energy charge-neutral excitation

where $\hbar \omega_{1}$ represents the incident photon energy before and $\hbar \omega_{2}$ the scattered photon energy after the scattering event. $E_{i}, E_{m}$ and $E_{f}$ denote the energies of initial $(i)$, intermediate $(m)$ and final $(f)$ states of the system, respectively. $d \Omega$ is the solid angle element and $\Gamma_{m}$ the core-hole life time width of the intermediate states. In Eq. (2.9) the first term in the sum over all final states $f$ describes elastic $\mathrm{X}$-ray scattering. The two contributions in the sum over all intermediate states $m$ are due to inelastic X-ray scattering, where the first and second term in this sum represent resonant and non-resonant (anomalous) inelastic X-ray scattering, respectively.

$3 \mathrm{~d}$ transition metal oxides, such as vanadium and copper oxides, are regarded as strongly correlated materials. In these systems, the electronic structure is characterized by the dual nature of delocalized and localized states $[1,7]$. Localized atomic like intermediate states are reflected by the occurrence of discrete charge-neutral low energy excitations (e.g. crystal field excitations) in the RSXE spectra $[11,12]$. These localized low-energy excitations are described by localized theoretical models like the single impurity Anderson model [11,12] (described in great detail in Sec. 3.4 of the next chapter) employing the resonant term of the Kramers-Heisenberg formula Eq. (2.9) which guides resonant inelastic X-ray scattering (RIXS). Figure 2.4 visualizes the one-step nature of RIXS by an energy level diagram.

Because of the involvement of two (virtual) dipole transitions the final state has the same parity as the initial state, and therefore crystal-field excitations between $d$-orbitals of different symmetry (so called $d d$-excitations) are allowed. Thereby, 
the electronic structure can be characterized in terms of $d d$-excitations and chargetransfer (CT) excitations, which arise due to transitions between delocalized ligand and localized transition metal atom states $[11,12,22,23]$. The excited electron in RIXS of correlated materials is localized at the excitation site, which is different than in wide-band materials, where the excited electron can dissipate to delocalized parts of the intermediate states.

For the delocalized part of the electronic structure in correlated materials similar dynamical dissipation processes are expected and therefore these components give rise to spectral features which are similar to $\operatorname{NSXE}[11,24,25]$ and will be referred to as ordinary fluorescence in this thesis. Energy conservation applies to the whole scattering process, and one obtains information about the ground state of the system via intermediate core excited states. The energy conservation requires that

$$
\hbar \omega_{2}=\hbar \omega_{1}-E_{e x c}
$$

where $\hbar \omega_{2}$ and $\hbar \omega_{1}$ are the energies of the scattered and incident photons, respectively. $E_{e x c}$ is the excitation energy of the final-state and corresponds to a localized low-energy charge-neutral excitation (See FIG. 2.4). Remarkably, the energy of the intermediate state does not enter in Eq. (2.10), which implies that the spectral broadening is not related to the life time of the intermediate state [10] as in NSXE. Thus, the spectral resolution is determined only by the experimental resolution, i.e. from the excitation source (width of $\hbar \omega_{1}$ ) and the spectrometer resolution.

\subsection{X-ray absorption spectroscopy}

X-ray absorption spectroscopy using synchrotron radiation is generally employed in two spectral regions, the spectral range of Near Edge X-ray Absorption Structure (NEXAFS) and the spectral range of Extended X-ray Absoprtion Fine Structure (EXAFS). A general and detailed description of the analysis of NEXAFS spectroscopy can for instance be found in the book by J. Stöhr [13]. In the soft X-ray range NEXAFS spectroscopy is a particular powerful chemical characterization technique which is often simply denoted as soft X-ray absorption (SXA) spectroscopy.

In SXA, a core electron is excited to an empty conduction band state. As such, SXA probes the unoccupied part of the electronic structure of the investigated system and thereby valuable information about the chemical composition can be gained. Due to the atomic nature of the core hole elemental and chemical state selectivity is obtained. If the incoming photon energy matches the energy required to promote a core electron to an unoccupied state an excitation may occur (see FIG. 2.1 (a)), i.e the atom may absorb the incoming photon. The probability of such a transition determines the X-ray absorption cross section and thus the absorbed X-ray intensity is governed by Fermi's Golden Rule:

$$
I_{S X A} \sim\left|\left\langle\Phi_{f}|\hat{e} \cdot r| \Phi_{i}\right\rangle\right|^{2} \delta_{E_{f}-E_{i}-\hbar \omega}
$$


The intensity $I_{S X A}$ in (1) is proportional to the squared diploe matrix element $(\hat{e} \cdot r)$ between initial state $\Phi_{i}$ and final state $\Phi_{f}$. The delta function assures energy conservation in that the difference between the energies of final $\left(E_{f}\right)$ and initial $\left(E_{i}\right)$ state have to be equal to the absorbed photon energy $\hbar \omega$.

In the soft X-ray range quadrupole transitions are some hundred times weaker than dipole transitions and therefore the SXA process is governed by dipole selection rules. This entails that the allowed transitions are restricted to transitions which change the angular momentum quantum number $(l)$ by one, $\Delta l= \pm 1$. In addition the z-component $(m)$ of the orbital momentum has to change by $\Delta m= \pm 1,0$ and the spin $(s)$ is conserved, $\Delta s=0$.

The core excited state can decay in two channels, nonradiative by the emission of an Auger electron or radiative by emitting a photon. In the soft X-ray region, the Auger electron yield is usually higher than 99\%, i.e. the X-ray fluorescence yield is often much less than $1 \%$. An avalanche of cascading excitations initiated by the Auger decay results in the creation low energy electrons that can emerge from the sample. The corresponding electron signal can either be detected by measuring the drain current or with a electron sensitive detector, e.g. a multi channel plate detector or a channel electron multiplier (CEM). By appropriate negative biasing of such a CEM detector also the fluorescence signal can be used for the SXA measurement. A positively biased grid is usually placed in front of the detector in order to reject ion contribution to the spectra.

The intensity of these secondary Auger electrons (electron yield) or the emitted photons (fluorescence yield) can be measured as a function of incoming photon energy. This will reflect the absorption cross section as the intensity of the secondary electrons/emitted photons is approximately proportional to the intensity of the absorbed incident X-ray photons. Because of the short mean free path of low energy electrons (about 50-200 ̊) using the total electron yield (TEY) signal is a relative surface sensitive X-ray absorption probe. If instead the emitted photons are employed for the x-ray absorption signal, i.e. SXA is recorded in fluorescence yield (FY), SXA can be made bulk sensitive with a probing depth of about 1000-2000 ^) due to the comparatively large attenuation lengths. However, when employing FY-SXA one is experimentally limited in the usable geometry regarding the incident X-rays due to possible self-absorption and saturation effects. Especially soft X-rays can easily be reabsorbed on the way leaving the material and thereby the relative spectral components can be distorted. 


\section{Chapter 3}

\section{Theoretical Models}

At the present time there exists no unified model for interpreting the electronic properties of transition metal oxides. There are two groups of opposing models of the electronic structure of solids, one type of models describes the electrons in a localized picture (ionic or cluster models) and another kind of models deals with extended or delocalized electron states (band theory) [1]. Band-like properties arise from a strong valence orbital overlap of atomic-like orbitals in a solid and therefore delocalized band theory accounts for the physical properties of most metals.

Electron correlations are many body effects that crucially influence the physics of many electronic systems. One most prominent example is electron correlation in solids where the Coulomb interaction between the electrons produces localization and thus is responsible for the peculiar transport properties of many transition metal oxides. Extreme localized models are ionic models, which concentrate on the properties of individual ions of crystal. One most prominent ionic model is the crystal-field model $[1,26]$, which describes how transition metal ions with $d^{n}$ configuration are perturbed by their chemical environment. In general it is not possible to strictly assign a particular transition metal oxide material to either of these two groups of models. Furthermore, one always needs to consider a competition between the different interactions, which either favor localization or delocalization of electrons.

\subsection{Crystal-field theory}

Crystal-field theory (CFT) [1,26] was developed by Hans Bethe in 1929 to account for the electronic structure and chemical properties of d-metal ions in ionic crystals. In CFT, a ligand-lone pair is idealized as a point negative charge repelling the $\mathrm{d}$ electrons in the orbitals of the central metal ion due to purely electrostatic interactions. The five d orbitals in an isolated gaseous metal are degenerate. If a spherically symmetric field of negative charges is placed around this metal ion, these orbitals remain degenerate, but all of them are raised in energy as a result of the repulsion between the negative charges on the ligands and in the d orbitals. 

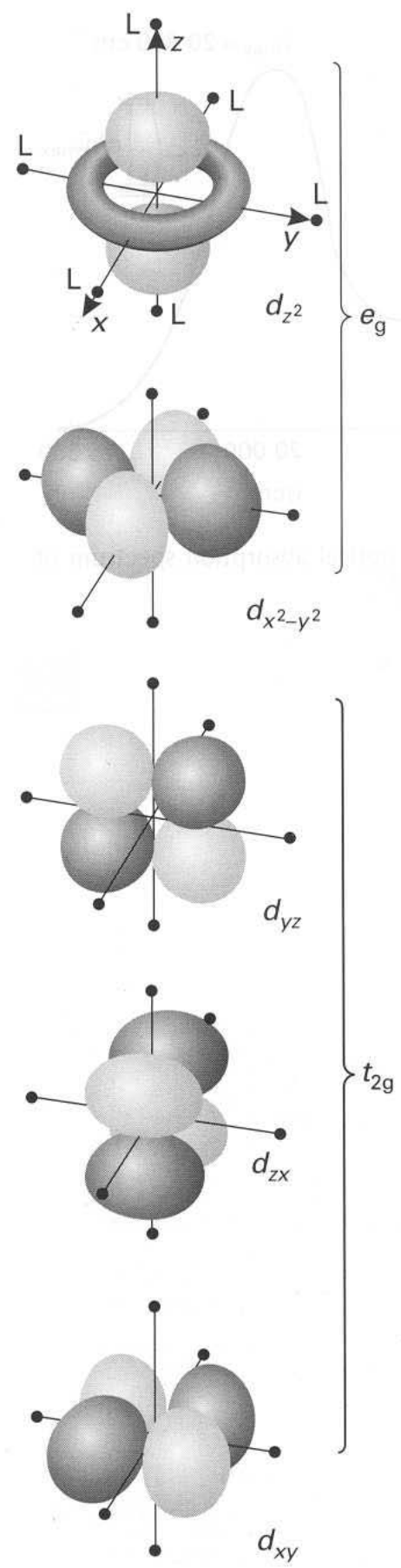

Figure 3.1: The orientation of the d orbitals of different symmetry in an octahedral crystal field. [Figure 7.8 (p. 227) from Inorganic Chemistry third edition by Shriver, D.F. and Atkins, P.W. (1999), by permission of Oxford University Press] 


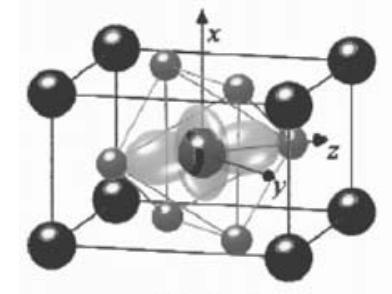

(a) $d_{3 z^{2}-r^{2}}$

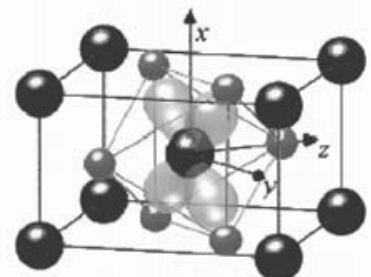

(b) $d_{x y}$

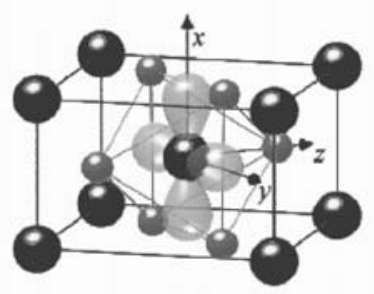

(c) $d_{x^{2}-y^{2}}$

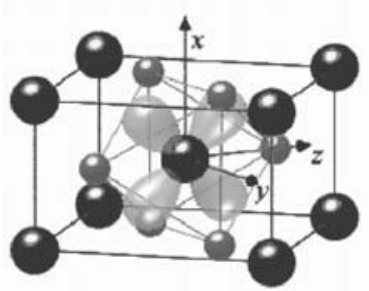

(d) $d_{x z}$

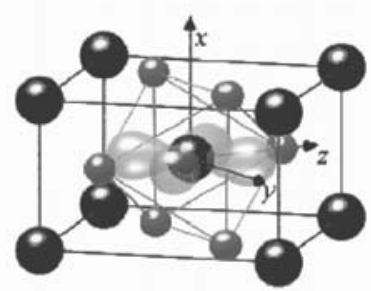

(e) $d_{y z}$

Figure 3.2: Angular parts of the d orbitals relative to the local reference frame of the central metal atom.[Reprinted with permission from Annalen der Physik (Leibzig), 10, Volker Eyert, The metal-insulator transition of $\mathrm{VO}_{2}$ : A band theoretical approach, 650-702, Copyright 2002, with permission from WILEYVCH Verlag $\mathrm{GmbH}]$

In case of a non-spherical symmetric crystal-field, the discrete point charges of the ligands interact differently with the d-metal ion orbitals, and thereby the degeneracy of the $\mathrm{d}$ orbitals is lifted. This model explains the splitting of the $\mathrm{d}$ orbitals in groups of different energies. The electrostatic energy is increased for d orbitals, which are oriented towards the region of high electron density; and likewise the energy is lowered when the opposite is the case. The total crystal field splitting energy may be estimated by

$$
D_{C F T} \approx \frac{r_{\text {orbital }}^{4}}{R_{\text {metal-ligand }}^{5}},
$$

where $r_{\text {orbital }}$ is the radius of the $\mathrm{d}$ orbital and and $R_{\text {metal-ligand }}$ is the metalligand internuclear distance. However, simple CFT ignores covalent interactions between the ligand and the central metal ion. Ligand-field theory accounts for this deficiency of CFT by including the overlap of $d$ and ligand orbitals in form of 


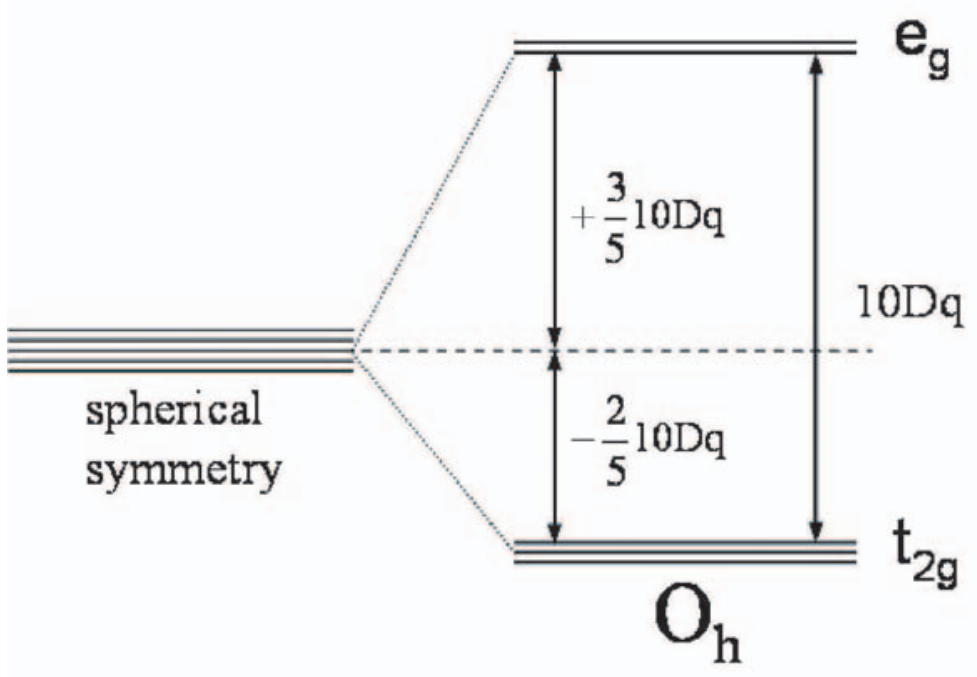

Figure 3.3: The energies of the d orbitals of a transition metal ion in an octahedral crystal field.

molecular orbitals.

In the CFT model of ionic complexes in octahedral $\left(O_{h}\right)$ symmetry, six ligands are placed at the corners of an octahedra around the central metal ion. Electrons in the two d orbitals of $e_{g}$ symmetry $\left(d_{z^{2}}\right.$ and $\left.d_{x^{2}-y^{2}}\right)$, pointing directly towards the ligands, are repelled more strongly by the negative charge of the ligands than electrons in the three $\mathrm{d}$ orbitals of $t_{2 g}$ symmetry $\left(d_{x y}, d_{y z}\right.$ and $\left.d_{z x}\right)$, pointing between the ligands (see FIG. 3.1). According to group theory the $e_{g}$ orbitals are doubly degenerate and the $t_{2 g}$ orbitals are triply degenerate. In the energy level diagram in FIG. 3.3 of this simple model the $e_{g}$ orbitals lie above the $t_{2 g}$ orbitals. The separation of these orbitals is expressed by the crystal-field splitting parameter $10 D q$. In a spherically symmetric environment the mean energy averaged over all $\mathrm{d}$ orbitals has to remain unchanged. Therefore the three $t_{2 g}$ and two $e_{g}$ orbitals lie $\frac{2}{5} 10 D q$ below and $\frac{3}{5} 10 D q$ above the average energy, respectively. The magnitude of $10 D q$ changes for different octahedral complexes. It depends on the identity of the metal ion, the charge on this ion, and the nature of the ligands coordinated to the metal ion.

When the crystal structure of a solid is constituted of basic structural units of distorted metal-oxygen octahedra, it is often a good starting point to analyze the electronic structure in $O_{h}$ symmetry. In this thesis the RSXE spectra of several vanadium oxides have been analyzed with a model employing $O_{h}$ symmetry. If, however, the distortion is large, the electronic structure has to be explained in lower symmetry groups. One prominent departure from a regular octahedron is the tetragonal distortion, where the Z-axis of the octahedron is elongated and the $\mathrm{X}$ - and Y-axes are compressed. In tetragonal symmetry $\left(D_{4 h}\right)$ the energy of the $d_{z^{2}}$ is reduced, whereas the energy of the $d_{x^{2}-y^{2}}$ is increased. Therefore a further 
energetic splitting of the $t_{2 g}$ and $e_{g}$ orbitals occurs. Other common distortions of six-coordinate complexes, which remove the orbital degeneracy, are square-planar and hexagonal.

\subsection{Band theory}

Modern band theory calculations of solids are generally based on the densityfunctional theory (DFT) [27]. In the DFT the eigenfunctions $\phi_{i}(\mathbf{r})$ are determined by the Kohn-Sham equation [28],

$$
\left[-\frac{\hbar^{2}}{2 m} \nabla^{2}+V_{p}(\mathbf{r})\right] \phi_{i}(\mathbf{r})=\varepsilon_{i} \phi_{i}(\mathbf{r})
$$

with the atomic periodic potential of the crystal lattice given by

$$
V_{p}(\mathbf{r})=v_{i o n}(\mathbf{r})+e^{2} \int d \mathbf{r}^{\prime} \frac{n\left(\mathbf{r}^{\prime}\right)}{\left|\mathbf{r}-\mathbf{r}^{\prime}\right|}+\frac{\delta E_{X C}[n(\mathbf{r})]}{\delta n(\mathbf{r})},
$$

where the electron density is connected to the eigenfunctions of the Kohn-Sham equation (3.2) by

$$
n(\mathbf{r})=\sum_{i}\left|\phi_{i}(\mathbf{r})\right|^{2}
$$

In Eq. (3.3) $E_{X C}[n(\mathbf{r})]$ is the exchange-correlation energy, and $v_{i o n}(\mathbf{r})$ represents the potential of the ion lattice, excluding the electrons under consideration. In the local density approximation (LDA) $E_{X C}[n(\mathbf{r})]$ is approximated by a homogeneous electron gas with the same density as the local density of the system:

$$
E_{X C}[n]=\int n(\mathbf{r}) \varepsilon_{X C}[n] d \mathbf{r} .
$$

Knowing $E_{X C}[n(\mathbf{r})]$, the Kohn-Sham Eq. (3.2) can be solved self-consistently to determine the electron density $n(\mathbf{r})$. The ground state electronic energy of the system can then be written as:

$$
\begin{array}{r}
E[n]=-\frac{\hbar^{2}}{2 m} \sum_{i} \int \phi_{i}^{*}(\mathbf{r}) \nabla^{2} \phi_{i}(\mathbf{r}) d \mathbf{r}+\int v_{i o n}(\mathbf{r}) n(\mathbf{r}) d \mathbf{r} \\
+\frac{e^{2}}{2} \int \frac{n(\mathbf{r}) n\left(\mathbf{r}^{\prime}\right)}{\left|\mathbf{r}-\mathbf{r}^{\prime}\right|} d \mathbf{r} d \mathbf{r}^{\prime}+E_{X C}[n] .
\end{array}
$$

Extending the LDA to inhomogeneous electron distributions can be accomplished by the corrections of the general gradient approximation (CGA) [29], where a correction energy is added to $E_{X C}[n(\mathbf{r})]$. The DFT is a powerful method for obtaining ground state properties, but is generally less accurate for excitations and for systems with strongly correlated electrons.

However, for systems with weak electron correlations (like wide band materials), the Kohn-Sham Eq. (3.2) reduces to the one-electron Schrödinger equation 
and can be employed in analysis of the electronic structure of such materials. In this work we compare the ordinary fluorescence part of the resonant soft X-ray emission spectra to partial density of states calculated from band structure calculations within the DFT scheme. This component of the electronic structure arises from delocalized intermediate states and is therefore only weakly effected by electron correlation effects similar as wide band materials.

\subsection{Hubbard Models}

In band theory, it is assumed that repulsion between electrons can be represented by using an average effective potential. In this approximation, the interaction of the independent electrons in the solid is described by a periodic potential and the mean field of the other electrons. For narrow valence band materials this simplification breaks down and such materials are difficult to describe with conventional band theory. Instead the on-site Coulomb interaction (U) between the electrons becomes comparable to the one electron band width (W) and a more localized way of describing their electronic properties is needed. Many transition metal oxides, like copper and vanadium oxides belong to this class of materials.

The electronic structure of such correlated systems can better be described by so-called Hubbard-like models. Such a Hubbard model [30] is based on the idealization that one can neglect the repulsion between electrons except when they are on the same atomic site. The electron correlation is then expressed by the on-site Coulomb interaction (U), a parameter which is often simply denoted as the Hubbard U. One simple form of a Hubbard Hamiltonian [30], which includes also the interaction with neighboring sites is

$$
H_{H u b b a r d}=-t \sum_{(i, j), \sigma}\left(c_{i \sigma}^{\dagger} c_{j \sigma}+c_{j \sigma}^{\dagger} c_{i \sigma}\right)+U \sum_{j} n_{j \uparrow} n_{j \downarrow},
$$

where $t$ is the transfer integral to neighboring sites, $(i, j)$ stands for the summation over all nearest neighbors, $c_{i \sigma}^{\dagger} c_{i \sigma}$ are creation and annihilation operators of electrons of spin $\sigma$ at site $i$, and $n_{i \sigma}=c_{i \sigma}^{\dagger} c_{i \sigma}$ gives the number operator for electrons with spin $\sigma$ at site $i$.

\subsection{Single impurity Anderson model}

For correlated electron systems with strong intra-atomic Coulomb interactions, like $3 \mathrm{~d}$ transition metal oxides or f electron materials, an atomic-like approach is necessary to characterize the electronic structure. In such a approach a simplified model Hamiltonian can be used that includes only the interactions around one specific metallic site. Therefore this kind of model is often called single impurity Anderson model (SIAM) $[11,12,22,23]$. The SIAM is related to the Hubbard model [30] in that it also accounts for correlation effects by an on-site Coulomb interaction parameter U. It was originally proposed for the description of magnetic moments of impurity transition metal 3d atoms in a nonmagnetic metal matrix by 
Anderson [31]. Later, the SIAM could widely be employed in the analysis of X-ray photoemission and X-ray absorption spectra of $d$ and f electron systems [32].

Recently, the SIAM has extensively been used in the interpretation of resonant soft X-ray emission (RSXE) and resonant inelastic X-ray scattering (RIXS) spectra $[11,12,22,23]$. In the SIAM the final state in RIXS of $d$ and $f$ electron systems can be described in terms of of intra-atomic excitations and inter-atomic charge transfer excitations. The intra-atomic excitations give the multiplet structure of the ground state electronic configuration due to electrostatic, exchange, crystal field and spin-orbit interactions. The inter-atomic charge transfer excitations, originating from an electron hopping mechanism from delocalized hybridized ligand states to localized metal states of the valence band, display the coupling of the localized metal ion states to the hybridized valence band levels of the ligands. The model Hamiltonian of the SIAM considers in addition to a single atom with d or f electron states also the extended valence and conduction band states, and the hybridization between localized metal and delocalized ligand states of the valence band.

One specific representation of a SIAM for transition metal oxides is the so called single transition metal site $M L_{6}(\mathrm{M}=$ metal atom, $\mathrm{L}=$ ligand atoms) cluster model in octahedral symmetry $\left(O_{h}\right)$ [33-35]. This model is especially useful for crystals of transition metal oxides consisting of basic building blocks of $M L_{6}$ clusters. In this thesis this kind of cluster model was employed to analyze the RSXE spectra of several vanadium oxides (see Sec. 5.1 in chapter 5). The Hamiltonian of the $M L_{6}$ cluster model is given by

$$
H=H_{\text {atom }}+H_{C F}+H_{L}+H_{m i x},
$$

where the first term $H_{\text {atom }}$ describes the atomic state of metal site $\mathrm{M}$ including $\mathrm{M}$ $2 \mathrm{p}$ and $\mathrm{M} 3 \mathrm{~d}$ states, and the second term $H_{C F}$ represents the crystal field splitting. The third and fourth terms, $H_{L}$ and $H_{m i x}$, describe the L $2 \mathrm{p}$ molecular orbital energy of the ligand and the hybridization between between M 3d states and L 2p molecular orbitals, respectively. All these terms are written as

$$
\begin{gathered}
H_{\text {atom }}=\sum_{\nu} \varepsilon_{d} d_{\nu}^{\dagger} d_{\nu}+\sum_{\mu} \varepsilon_{c} c_{\mu}^{\dagger} c_{\mu}+U_{d d} \sum_{\nu>\nu^{\prime}} d_{\nu}^{\dagger} d_{\nu} d_{\nu^{\prime}}^{\dagger} d_{\nu^{\prime}} \\
-U_{d c} \sum_{\nu, \mu} d_{\nu}^{\dagger} d_{\nu}\left(1-c_{\mu}^{\dagger} c_{\mu}\right)+H_{\text {multiplet }}, \\
H_{C F}=\sum_{\nu} \varepsilon(\Gamma) d_{\nu}^{\dagger} d_{\nu},
\end{gathered}
$$

with

$$
\begin{gathered}
\varepsilon(\Gamma)= \begin{cases}\frac{3}{5}(10 D q) & \text { for } \Gamma=e_{g} \\
-\frac{2}{5}(10 D q) & \text { for } \Gamma=t_{2 g},\end{cases} \\
H_{L}=\sum_{\nu} \varepsilon_{L} L_{\nu}^{\dagger} L_{\nu},
\end{gathered}
$$


and

$$
H_{m i x}=\sum_{\nu} V(\nu)\left(d_{\nu}^{\dagger} L_{\nu}+L_{\nu}^{\dagger} d_{\nu}\right) .
$$

The first and second term of the Hamiltonian $\mathrm{H}_{\text {atom }}$ in Eq. (3.9) express the one particle energies of the M $3 \mathrm{~d}$ and M $2 \mathrm{p}$ states, respectively. Correlation effects in form of the Coulomb interaction $\mathrm{U}_{d d}$ between $\mathrm{M}$ 3d electrons and the influence of the attractive core-hole potential $-\mathrm{U}_{d c}$ on the $3 \mathrm{~d}$ electrons are accounted for by the third and fourth term in $\mathrm{H}_{\text {atom }}$, respectively. The last term includes intra-atomic multiplet coupling and spin-orbit interactions for the M 3d and M 2p states.

In Eq. (3.9) - (3.13) $d_{\nu}^{\dagger}\left(d_{\nu}\right), c_{\mu}^{\dagger}\left(c_{\mu}\right)$ and $L_{\nu}^{\dagger}\left(L_{\nu}\right)$ are electron creation (annihilation) operators for M 3d, core M 2p, and L 2p orbitals, respectively. $\nu$ and $\mu$ represent combined indices for spin $(\sigma)$ and orbital quantum numbers $(\Gamma)$, where $\Gamma$ stands for $t_{2 g}$ and $e_{g}$, which are irreducible representations of $O_{h} . \varepsilon_{d}, \varepsilon_{c}$ and $\varepsilon_{L}$ are one particle energies for the M $3 \mathrm{~d}, \mathrm{M} 2 \mathrm{p}$ and L $2 \mathrm{p}$ states. $10 D q=\left[\varepsilon\left(e_{g}\right)-\varepsilon\left(t_{2 g}\right)\right]$ and $V(\Gamma)$ denote the energy of the crystal field splitting and the hybridization strength between $M 3 d$ and $L 2 p$ states, respectively. The charge transfer (CT) energy is defined as $\Delta=E\left(d^{n+1} \underline{L}\right)-E\left(d^{n}\right)$ with $n=0,1,2$, where $\underline{L}$ denotes a hole in the L $2 \mathrm{p}$ orbital, and $E\left(d^{n+1} \underline{L}\right)$ and $E\left(d^{n}\right)$ denote multiplet averaged energies of the respective $d^{n+1} \underline{L}$ and $d^{n}$ configurations. In calculations within the $M L_{6}$ cluster model $\Delta, U_{d d}, U_{d c}, V\left(e_{g}\right)\left[=-2 V\left(t_{2 g}\right)\right]$ and $10 D q$ are treated as adjustable parameters, which have to be optimized by fitting experimental data from core-level spectroscopies like RIXS.

The initial and final state of RIXS, $|g\rangle$ and $|f\rangle$, are described by a linear combination of three configurations, which are mixed with each other by hybridization:

$$
\begin{gathered}
|g\rangle=c_{0}\left|d^{n}\right\rangle+c_{1}\left|d^{n+1} \underline{L}\right\rangle+c_{2}\left|d^{n+2} \underline{L}^{2}\right\rangle \\
|f\rangle=c_{0}^{\prime \prime}\left|d^{n}\right\rangle+c_{1}^{\prime \prime}\left|d^{n+1} \underline{L}\right\rangle+c_{2}^{\prime \prime}\left|d^{n+2} \underline{L}^{2}\right\rangle .
\end{gathered}
$$

The final state of X-ray absorption (XA) spectroscopy at M 2p-edges is the intermediate state $|m\rangle$ of RIXS, when a M 2p core electron is excited to an empty M 3d orbital, and can be written as follows:

$$
|m\rangle=c_{0}^{\prime}\left|\underline{\mid} d^{n+1}\right\rangle+c_{1}^{\prime}\left|\underline{c} d^{n+2} \underline{L}\right\rangle+c_{2}^{\prime}\left|\underline{c} d^{n+3} \underline{L}^{2}\right\rangle .
$$

In the last Eq. (3.16) $\underline{c}$ symbolizes the hole in the M 2p core level. Diagonalizing the total Hamiltonian in Eq. (3.8) in the basis given by Eq. (3.14) - (3.16) gives all the eigenvalues and eigenfunctions of the system. The X-ray absorption spectrum (XAS) can then be calculated by

$$
F_{X A S}(\hbar \Omega)=\sum_{m}|\langle m|D| g\rangle|^{2} \delta\left(\hbar \Omega-E_{m}+E_{g}\right),
$$

where $\hbar \Omega$ stands for the incident photon energy, and $|g\rangle$ and $|m\rangle$ represent the ground and the final state in XAS with the respective energies $E_{g}$ and $E_{m}$. The RIXS spectrum is calculated by the resonant term of the Kramers-Heisenbergequation (2.9), which is written as 


$$
F_{R I X S}(\hbar \Omega, \hbar \omega)=\sum_{f}\left|\sum_{m} \frac{\langle f|D| m\rangle\langle m|D| g\rangle}{E_{m}-E_{g}-\hbar \Omega-\frac{i}{2} \Gamma_{m}}\right|^{2} \times \delta\left(\hbar \Omega-\hbar \omega+E_{g}-E_{f}\right)
$$

where $|g\rangle,|m\rangle$ und $|f\rangle$ represent ground, intermediate and final states in RIXS with the energy eigenvalues $E_{g}, E_{m}$ and $E_{f}$. D stands for the electric dipole transition operator. The photon energy loss (X-ray Raman shift) between incident photon energy $\hbar \Omega$ and emitted photon energy $\hbar \omega$ is $\hbar \omega-\hbar \Omega$. 



\section{Chapter 4}

\section{Experimental description}

\subsection{Synchrotron radiation sources}

In order to perform RSXE spectroscopy, one needs a tunable soft X-ray light source with high photon flux. Conventional laboratory X-ray light sources like $\mathrm{X}$-ray tubes or rotating anodes consist of a solid target which is bombarded with high-energy electrons to create X-rays in form of bremsstrahlung and characteristic radiation [36]. These kinds of X-ray sources are limited in achievable photon flux due to cooling restrictions, which arise when a large portion of the incident kinetic electron energy is dissipated as heat. Other interesting, though technically more complicated soft X-ray sources, like for instance plasma X-ray sources [36, 37], are promising candidates for future applications. Therefore modern RSXE experiments have to be performed at synchrotron radiation sources [36,37].

An accelerated charged particle emits electromagnetic radiation [38]. When such a particle is moving at relativistic velocities, the emitted radiation is concentrated in a narrow cone tangentially to the path of it $[39,40]$. Synchrotron radiation $[36,37]$ is generated when relativistic electrons (or positrons) are centripetally accelerated by the Lorentz force of strong magnets in a curved trajectory. In a storage ring a series of magnets are utilized to force the electrons on a circular path at nearly constant relativistic velocities. The loss of energy of the electron beam due to the emitted synchrotron radiation is compensated by a oscillating radio frequency (RF) electric field in so called RF-cavities. Commonly three types of magnetic structures $[36,37]$ are used to produce radiation in modern synchrotron facilities: dipole magnets, undulators, and wigglers (see FIG. 4.1).

Dipole magnets "bend" the electrons in a single curved trajectory in order to produce a narrow fan of radiation of continuous wavelength around the center of the dipole. Undulators are periodic magnetic structures, in which the electrons are forced to follow a path of a harmonic oscillation. The resultant radiation is emitted in an extremely small radiation cone with very small angular divergence and spectral width. Wigglers operate with much higher magnetic fields as undulators. Stronger magnetic fields result in larger oscillation amplitudes and increased radiation power. Consequently, the radiation cone is broader in space and angle, 

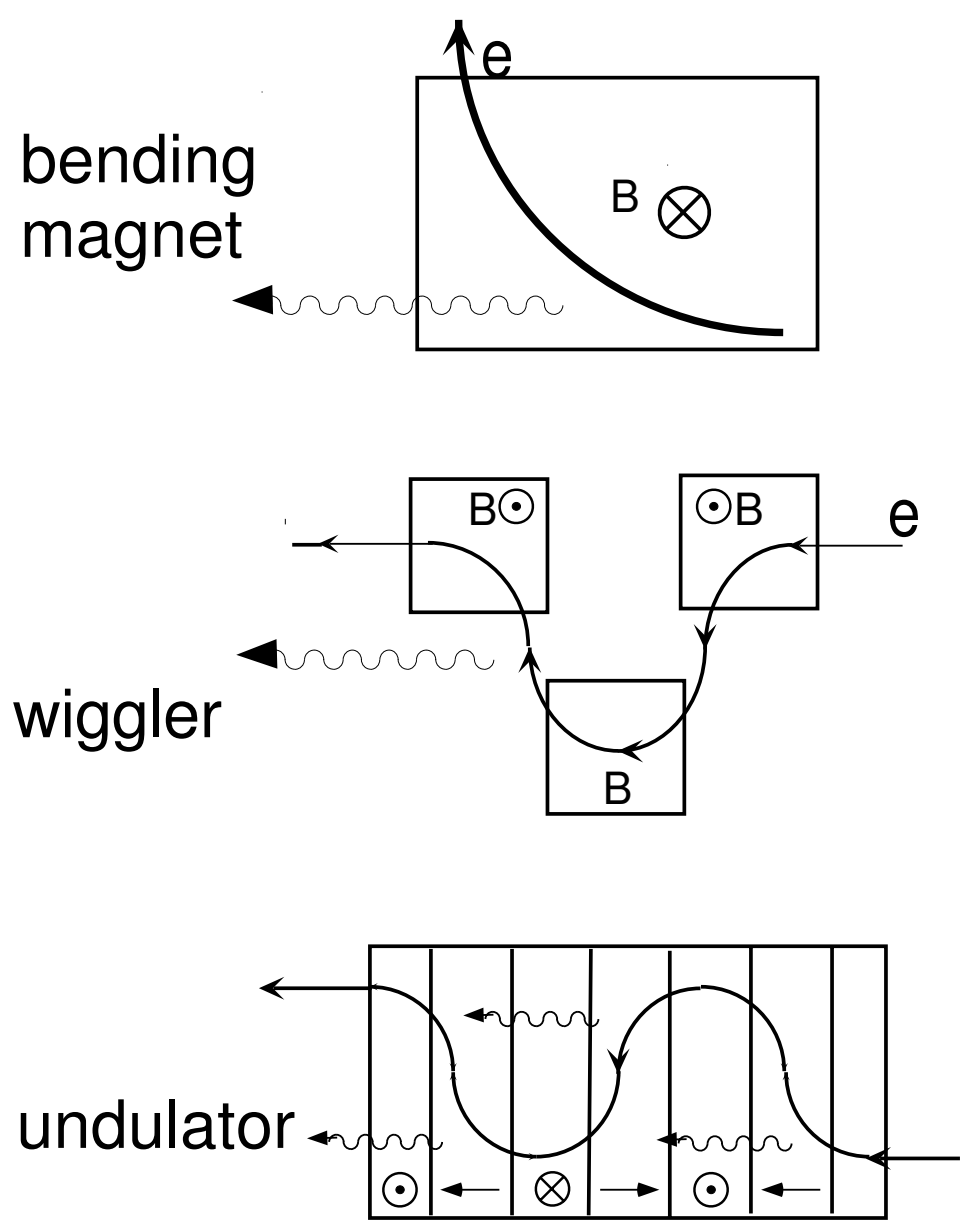

Figure 4.1: Working principles of the magnetic structures at a third generation synchrotron radiation ring: bending (dipol) magnet, wiggler and undulator [41].

and the emitted spectrum is similar to that of the bending magnet, but with much higher photon flux and a shift to shorter wavelength.

All experiments presented in this work have been performed with soft X-rays from undulators [36,37], which are installed in straight sections of the storage ring (which consists of circular and straight sections). The period of the alternating magnet pairs of opposite polarity in an undulator (see 4.2) is optimized in such way that the oscillating electrons emit coherent radiation pulses at each magnetic pole which can constructively interfere. This can be achieved by adapting the period length of the harmonic path of the electrons in the undulator in the desired emitted wavelength region. Therefore an undulator spectrum consists of sharp peaks, called harmonics. The energy position of these harmonics in an undulator spectrum depends on the magnetic field strength, which can be varied by changing the distance (gap) between the upper and lower row of permanent magnets of 


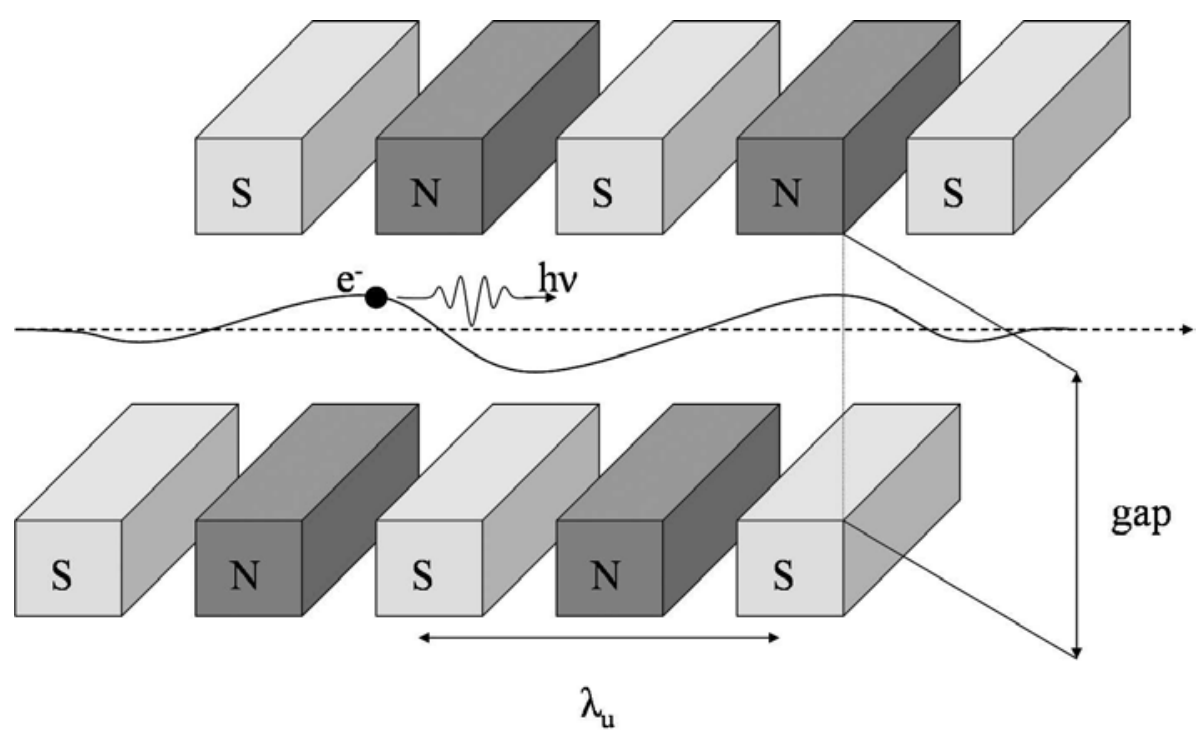

Figure 4.2: Schematic side view of an undulator.

alternating magnetic polarity (see 4.2). This design characteristic of undulator devices can be employed to get the desired tunability of the synchrotron radiation. The high flux of radiation from an undulator is thus concentrated in a narrow photon energy range of a few electron volts and is highly directional, which entails that a large portion of the total flux is available for the anticipated experiments. In a usual configuration undulators deliver linear polarized light with the polarization direction in the plane of the storage-ring. The wavelength of the radiation emitted from the undulator is determined by the extended undulator equation [37]

$$
\lambda_{n}=\frac{\lambda_{u}}{2 n \gamma^{2}}\left(1+\frac{K^{2}}{2}+\gamma^{2} \theta^{2}\right),
$$

where $\lambda_{u}$ is the period of the magnetic poles, $n$ the harmonic number $(n=$ $1,2,3 \ldots)$, the Lorentz contraction factor $\gamma=\frac{1}{\sqrt{1-\frac{v^{2}}{c^{2}}}}$, and the non-dimensional undulator parameter $K$ is related to the magnetic flux density $B$ by

$$
K=\frac{e B \lambda_{u}}{2 \pi m c}
$$

The third term in the undulator equation (4.1) depends in the solid angle of the central radiation cone of relative spectral bandwidth $\frac{\Delta \lambda}{\lambda}=\frac{1}{N n}$ written by

$$
\theta=\frac{\sqrt{1+\frac{K^{2}}{2}}}{\gamma \sqrt{N}} .
$$

The dependence of Eq. (4.3) illustrates that undulator spectra become more narrow, when the number of magnetic pairs $\mathrm{N}$ is increased. Higher harmonics $(n>1)$ 
occur due to transverse oscillations of the electron beam and extend the spectral region covered to shorter wavelengths [37]. The relative spectral bandwidth is also diminished by a factor $\frac{1}{n}$ for higher harmonics. Even harmonics, i.e. $n=2,4,6, \ldots$ have a radiation pattern that is extremely weak on axis. Therefore the only relevant harmonics for the use of undulator radiation in experiments are the odd harmonics $n=1,3,5, \ldots$, which radiate on axis. The wavelength tunability of an undulator is realized by varying the undulator parameter $K$ by changing the magnetic flux density $B$, i.e. the magnet gap.

\subsection{Beamlines}

In order to take advantage of the emitted synchrotron radiation in performing experiments, it has to be guided to a specialized experimental station, where samples are illuminated and investigated. For RSXE spectroscopy experiments it is also necessary to limit the spectral bandwidth of the radiation hitting the sample, i.e. to monochromatize. In the case of an undulator the synchrotron light is already quite narrow with a absolute width in the order of some electron volts, depending on the tuned energy and the used undulator harmonic. All this is accomplished by a beamline [42], which consists of a series of high precision optical elements, which direct, monochromatize and focus the radiation onto the sample.

These optical components have to be kept under ultra-high vacuum $\left(\sim 10^{-10}\right.$ mbar) in order to avoid attenuation of the photons by air molecules along the beamline and to minimize photochemical processes, which can lead to carbon and oxygen contamination of the surfaces of these elements. Carbon and oxygen containing contaminations of the optical elements generally lead to so called carbon and oxygen dips in the intensity response function of the beamline around $290 \mathrm{eV}$ and $530 \mathrm{eV}$, respectively.

Monochromatization of photons in the soft X-ray range is commonly accomplished by diffraction from reflection gratings $[36,42]$. The absorption of soft X-ray light by materials is relatively high and correspondingly the reflection at normal incidence extremely low. However, since the index of refraction is less than unity for all materials in the soft X-ray range total external reflection can be achieved, if sufficiently small incidence angles relative to the surface are employed. Therefore all mirrors and gratings, which are used as optical elements in a soft X-ray beamline have to be used at grazing incidence angles, i.e. at glancing angles smaller than the angle of total external reflection. Generally, the type of grating monochromators is classified by the shape of the used gratings. Nowadays, plane grating monochromators (PGM) and spherical grating monochromators (SGM) are the most common designs [42].

The experimental work presented in this thesis has mainly been performed at two undulator beamlines, at beamline 7.0.1 [43] at the Advanced Light Source (ALS) at Lawrence Berkeley National Laboratory (in Berkeley, California, USA), and at beamline I511-3 [44] at the MAX II storage ring at MAX-lab National Laboratory at Lund University (in Lund, Sweden). The principle characteristics of these beamlines and the used experimental stations will be described in the following. 


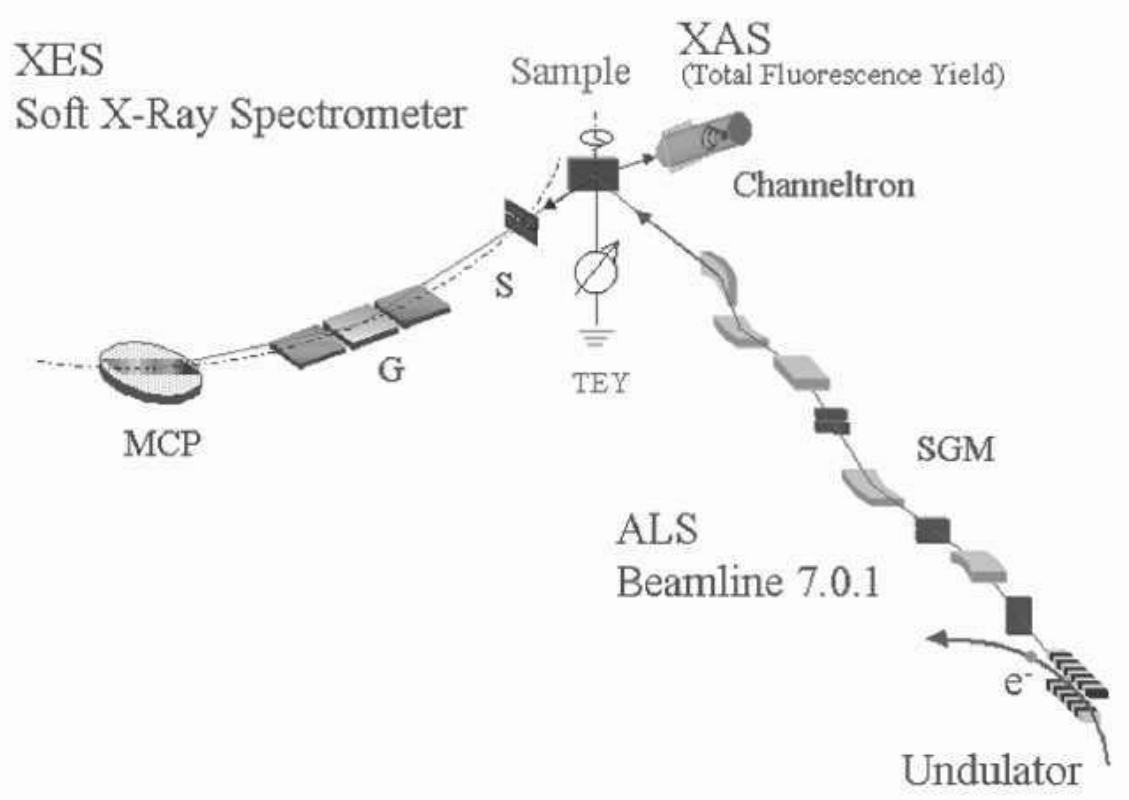

Figure 4.3: Schematic picture of beamline 7.0.1 at ALS and the experimental set-up used for soft X-ray spectroscopy [45].

\subsubsection{Beamline 7.0.1 at ALS}

The schematic layout of beamline 7.0.1 at the ALS is shown in FIG. 4.3 [45]. This beamline comprises a $5 \mathrm{~m}$ long undulator with 89 magnetic periods of a period length of $\lambda_{u}=50 \mathrm{~mm}$ [43]. It is equipped with a SGM monochromator in a nearly Rowland circle geometry with fixed entrance and translating exit slit covering an energy range of 60-1300 eV with three interchangeable gratings.

In the SGM concept [42] a pre-focussing mirror collects and focuses light from the undulator, limited to the acceptance angle of the central cone by horizontal beam defining apertures, onto the fixed entrance slit of the monochromator. The entrance slit defines the illuminated area of the spherical grating. At the grating the light is diffracted according to the grating equation (see Eq. 4.4 in Sec. 4.3). In order to focus a desired wavelength onto the movable exit slit the grating needs to be slightly rotated. A plane mirror situated after the exit slit directs the light to the used experimental end station. For most applications it is beneficial to illuminate only a small sample spot with synchrotron radiation of high photon flux and therefore the light is refocussed by two additional spherical mirrors in horizontal and vertical direction before striking the sample situated in the focal point of this refocussing mirror giving an illuminated beam spot of $40-50 \mu \mathrm{m}$.

The resolving power of the monochromator can be varied by adapting entrance and exit slit widths with a maximum achievable resolving power of $R=\frac{\lambda}{\Delta \lambda}=$ 10000 [43]. In the work presented in this thesis the beamline was operated in 
the 5th harmonic of the undulator employing the "high-energy" grating with 925 lines/mm with a resolving power of $R=\frac{\lambda}{\Delta \lambda} \approx 1700$, corresponding to a spectral resolution in the order of $0.3 \mathrm{eV}$ in the range of $500-550 \mathrm{eV}$. The undulator gap can be scanned coordinated together with the energy scanning of the monochromator in order to employ always the center of the undulator peak for the experiments.

The end station, in which all the measurements are performed, is equipped with a soft X-ray emission spectrometer and a channel electron multiplier, which can be used for total fluorescence yield absorption measurements. Total electron yield absorption can be measured by recording the drain current.

\subsubsection{Beamline I511-3 at MAX-Lab}

At beamline I511 a 49 period undulator with an undulator period length of $\lambda_{u}=52$ $\mathrm{mm}[44]$ is employed as light source providing linearly polarized photons in the horizontal plane of the laboratory. Monochromatization of the light is achieved by a PGM type monchromator [42] of the Zeiss SX700 design covering an effective energy range of $100-1200 \mathrm{eV}$ with one standard grating of 1220 lines $/ \mathrm{mm}$. In this design the beamline does not need a entrance slit, if the electron beam position in the storage ring is of high stability.

The undulator light is collected and horizontally focussed by a cylindrical premirror towards the monochromator, which consists of three optical elements. Before illuminating the plane grating the light is further redirected by a plane mirror, and after the grating the diffracted light is focused by a spherical mirror towards the exit slit. The required photon energy is selected by rotating the plane grating and thereby changing the wavelength, which is dispersed perpendicular to the exit slit. The resolving power of the monochromator is adjustable through the exit slit widths with a maximum achievable resolving power of $R=20000$ [46] for low energies. For the work presented in this thesis the measurements at I511-3 were performed in an energy range of ca. 500-550 eV employing the 3th harmonic of the undulator and using similar resolving power as at beamline 7.0.1 (ALS), i.e. with $R \approx 1700$.

Behind the exit slit beamline I511 splits into two branches with specialized end-stations. The branch used in the presented investigations, I511-3, is dedicated to bulk-sensitive spectroscopy of solids and liquids. The end-station has similar measurement equipment as the end-station at beamline 7.0.1 at ALS and is depicted in FIG. 4.4. A flippable mirror situated directly behind the monochromator exit slit is used to direct the light into one of the end stations. Both branch lines are equipped with refocusing optics, consisting of a so called Kirkpatrick-Baez pair of bendable plane-elliptical mirrors giving a beam spot size at the sample of ca. $20 \mu \mathrm{m} \times$ ca. $30 \mu \mathrm{m}[47]$ in vertical and horizontal direction, respectively.

\subsection{Soft X-ray emission spectrometer}

The resonant soft X-ray emission and resonant inelastic X-ray scattering spectra, presented in the investigations of this thesis, were measured with a Rowland mounted grazing incidence soft X-ray emission spectrometer [48]. The spherical 


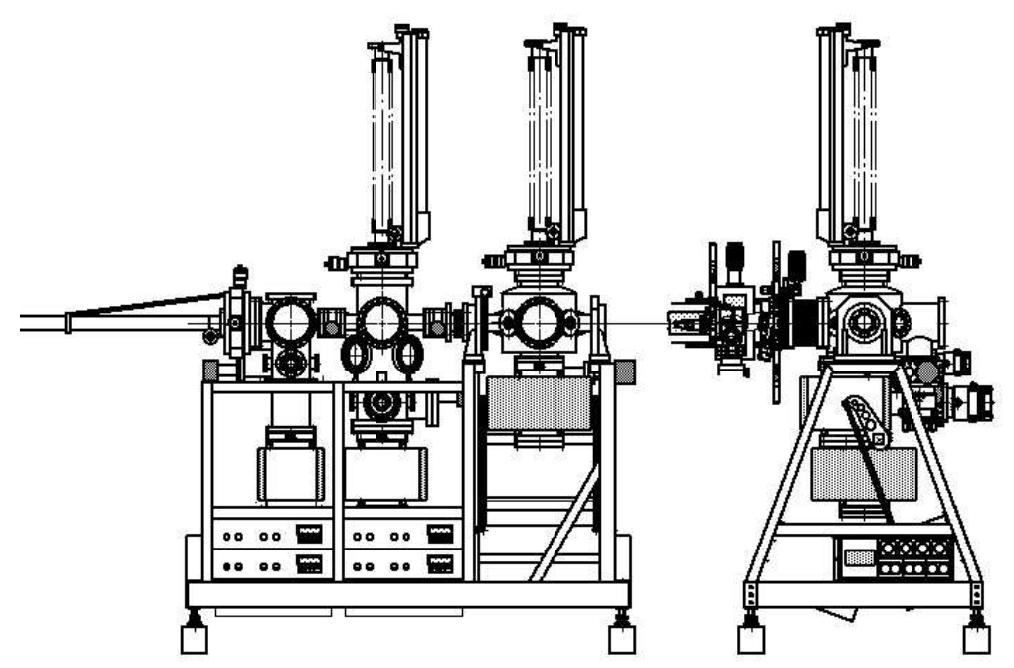

Figure 4.4: The enstation of beamline I511-3 at MAX-Lab. The view to the right is along the beam and shows in the front the analysis-chamber with the soft X-ray emission spectrometer directing to the left. The spectrometer is rotatable around the beam-axis. The left hand panel views the station from the side, so that all three chambers, the analysis chamber (right), the preparation chamber (middle) and loadlock (left) for a fast changing of samples can be seen [41].

diffraction grating of a Rowland type spectrometer achieves both the wavelength dispersion and the focusing in a single step [49]. The grating usually consists of a piece of glass coated with a high-Z metal like gold or platinum with a large number of parallel grooves. Light of different wavelengths, $\lambda$, is diffracted differently according to the grating equation

$$
n \lambda=d(\sin \alpha-\sin \beta),
$$

where the grating constant $d$ is the distance between two grooves, $n=0, \pm 1, \pm 2, \ldots$ stands for the different orders of diffraction, and $\alpha$ and $\beta$ are the angle of incident and diffracted light, respectively. The ultimate resolution of a grating depends on the total number of illuminated grooves and the diffraction order.

In a Rowland type spectrometer, focusing is achieved if source and detector are placed together with the spherical grating on a circle with half the radius of the grating $[42,49]$. However, this focussing condition for a spherical surface is only strictly fulfilled for the tangential point of grating and Rowland-circle. Therefore image distortions, i.e. so called aberrations, can decrease the resolution. The dominating abberation for a Rowland spectrometer in grazing geometry is the astigmatism, which results in imaging of a source point at the slit onto a curved line at the detector. Because of spherical abberation and coma the resolution of a Rowland grating spectrometer is also dependent on the illumination of the grating, and in order to achieve maximal possible resolution a grating should not be illuminated at larger widths than a certain optimal illumination width. Geometrical 


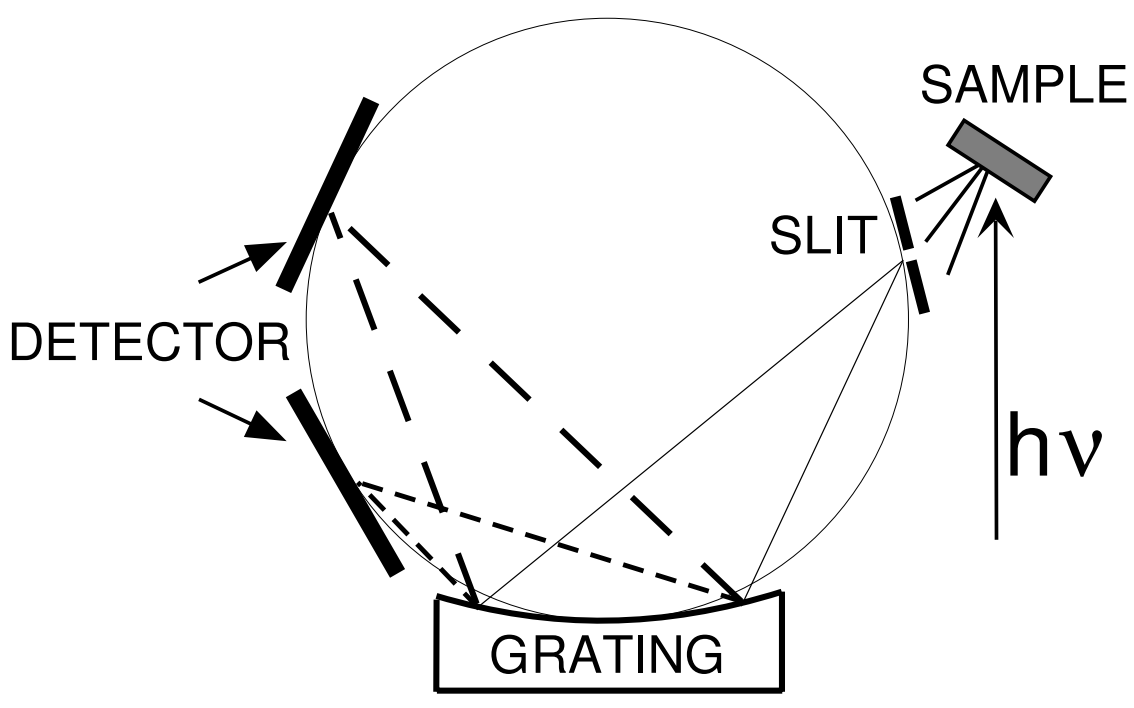

Figure 4.5: Rowland type design of a spectrometer with a spherical diffraction grating. The light is imaged from the slit via the grating onto the detector, which can record a certain wavelength range simultaneously. Two shown detector positions correspond to the foci of different wavelength intervals [41].

considerations and the diffraction limitation limit the wavelength resolution to

$$
\Delta \lambda=1.1 \frac{w d}{n R},
$$

where $w$ is the slit width, $d$ the grating constant, $n$ the diffraction order, and $R$ the grating radius.

A schematic picture of the soft X-ray emission spectrometer developed by J. Nordgren et al. [48] in the research group, in which the present work has been performed at Uppsala University, is depicted in FIG. 4.6. Three gratings, optimized for different energy ranges, are fix-mounted together with the entrance slit on Rowland circles of different radii. In order to have the joint fixed slit as a common point for the three different Rowland circles, the gratings are mounted at different angles of incidence. Two spherical gratings of 5 and one of 3 meters radius posses different groove densities of 1200, 400 and 300 lines $/ \mathrm{mm}$, as well as different grazing incidence angles $(1.9,2.6$ and 5.4), respectively. Together, these three gratings cover an energy range of $50-1000 \mathrm{eV}$.

The resolution of the instrument can be adapted by the entrance slit width, which is possible to vary continuously between 0 and ca. $120 \mu \mathrm{m}$. This entrance slit consists of two displaced precision knife-edges, which are rotatable around the symmetry axis in order to change the projected slit size towards the beam spot on the sample (see Ref. [50] for details). Since three gratings are mounted in the same spectrometer, grating selectors are used to define the illumination of the respective gratings. By using two separate (upper and lower) grating selectors the illuminated area of the gratings can be changed, between an optimum illumination 


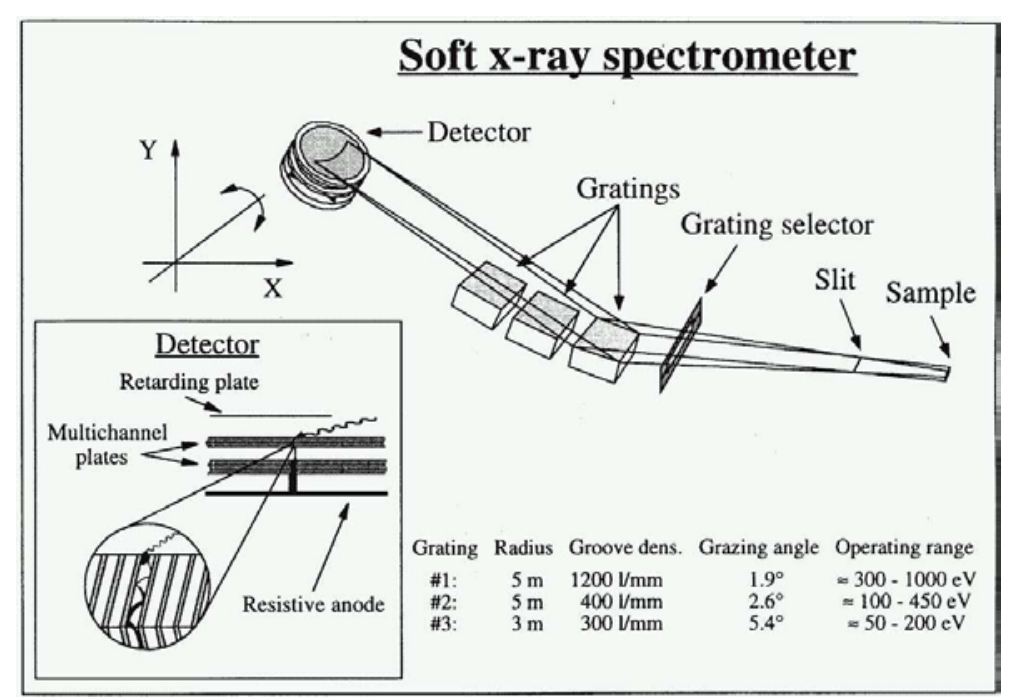

Figure 4.6: The grazing incidence grating spectrometer in Rowland circle geometry.

and larger illuminations, if a trade-off between resolution and throughput intensity is necessary.

The used two-dimensional detector system is situated in a housing, which comprises a stack of five multichannel plates (MCPs) of $40 \mathrm{~mm}$ diameter at ca. -4000 $\mathrm{V}$ and a resistive anode with position-sensitive readout. The detector housing is mounted in such a way that it can be moved with translational stepper motors along two perpendicular spatial axes $\mathrm{X}$ and $\mathrm{Y}$. The detector can also be rotated in the housing around an axis parallel to the detector surface in order to place the detector surface tangentially to the Rowland-circle. By this combination of three degrees of freedom the detector is positioned at different focus points on the Rowland-circle depending on the employed grating and the desired covered wavelength window. The efficiency of the detector is increased by coating the first MCP with a $3000 \AA$ A layer of CsI. A negatively biased front retarding electrode prevents that electrons created at the CsI surface and between the channels are not being registered by forcing them into the channels. In the CsI layer the photons are converted into electrons, which are multiplied in the channels of the MCPs.

After passing the whole stack of MCPs a multiplied electron cascade hits the resistive anode and the coordinates of the photon event can be determined by a position analyzer. The resulting two dimensional spectrum is divided in 32 slices perpendicular to the dispersion direction and 1024 channels in the dispersion direction. In order to convert the two dimensional spectrum into an ordinary one dimensional spectrum, the slices of single spectra have to be aligned by shifting them in the dispersion direction, before they are summed up. Thereby, it is possible to compensate for the astigmatism, which images the straight entrance slit of the spectrometer as a curved line at the detector. The spatial resolution of the detector system $(100 \mu \mathrm{m})$ does not limit the total resolution of the spectrometer considerably for slit widths larger than about $10 \mu \mathrm{m}$. 


\subsection{Sample preparations}

\subsubsection{Single crystals of vanadium and copper oxides}

All spectroscopic investigations on vanadium and copper oxides presented in this thesis have been performed on single crystals. Crystals of $\mathrm{VO}_{2}$ (used in paper II and XI), Mo doped $\mathrm{VO}_{2}$ (paper VI), $\mathrm{V}_{2} \mathrm{O}_{3}$ (paper II and VI) and $\mathrm{V}_{6} \mathrm{O}_{13}$ (paper VIII) were grown by Dr. M. Klemm (Universität Augsburg). These single crystals were grown at suitable growth temperatures in a gradient furnace by chemical transport reactions [51,52], which use $\mathrm{TeCl}_{4}$ as transport agent [53]. X-ray diffraction was used to check the purity of the synthesized crystals and to verify that the samples consist only of one single crystal phase. For vanadium oxides that exhibit a metal-insulator transition standard electrical resistivity and magnetic susceptibility measurements were employed to determine the respective transition temperatures. Typically, single crystals of a few $\mathrm{mm}^{3}$ were used in the spectroscopic investigations.

Crystals of $\mathrm{NaV}_{2} \mathrm{O}_{5}$ (paper II, VI and XV) were grown by A. Revcolevschi and G. Dhalenne (Université de Paris) by a flux method. In this technique appropriately compacted mixtures of $\mathrm{V}_{2} \mathrm{O}_{5}, \mathrm{~V}_{2} \mathrm{O}_{3}$ and $\mathrm{NaVO}_{3}$ in platinum crucibles are melted under vacuum, and by subsequently slow cooling of these melts from 1073 $\mathrm{K}$ to room temperature similarly as described by Isobe and Ueda [54] plate-shaped crystals are obtained. Fibers of $\mathrm{Na}_{2} \mathrm{~V}_{3} \mathrm{O}_{7}$ nanotubes (paper XII) were grown in a tubular furnace from melts with a starting composition of $\mathrm{Na}_{1.9} \mathrm{~V}_{2} \mathrm{O}_{5}$ by P. Millet (Centre d'Elaboration de Matériaux ed d'Etudes Structurales, Toulouse) [55]. $\mathrm{CuGeO}_{3}$ (paper I, III and VII) and $\mathrm{SrCuO}_{2}$ (paper III, VII and XIV) single crystals of several cm length were grown by A. Revcolevschi and G. Dhalenne (Université de Paris) from the melt by a floating zone technique [56]. The used $\mathrm{CuO}$ and $\mathrm{La}_{2} \mathrm{CuO}_{4}$ samples (paper III and VII) were commercially available single crystals.

\subsubsection{Vanadium oxide battery cathodes}

The objective of papers IV, V, IX and X of this thesis is to study the electronic structure of vanadium oxide used as cathode materials in a electrochemical battery cell. For these investigations electrochemical insertion of lithium has been performed outside vacuum into cathodes consisting of vanadium oxides. All battery cathode samples were prepared at the Department of Materials Chemistry (Ångström laboratory). The samples were then transported in sealed aluminum bags to beamline I511-3 (MAXLAB). Before carrying out the soft X-ray spectroscopy experiments, the sample holders with the cathodes were loaded into the vacuum via a load-lock chamber. During loading, a glove-bag was tightly attached to the load-lock port under argon-gas flow. Sample contact with oxygen or moisture from the room atmosphere was therefore minimised, so that oxidation of the battery cathodes was avoided. The samples were transferred from the load-lock chamber into a measuring chamber for the soft X-ray spectroscopy experiments with a base pressure of $1 \times 10^{-9}$ mbar. Two types of vanadium oxide materials were studied: $\mathrm{V}_{6} \mathrm{O}_{13}$ (paper IX and $\mathrm{X}$ ) and vanadium oxide nanotubes (paper IV and V). 
$\mathbf{V}_{6} \mathbf{O}_{13}$

Polycrystalline powder of $\mathrm{V}_{6} \mathrm{O}_{13}$ was prepared as described by Lampe-Önnerud and Thomas [57]. A cathode slurry $\left(\mathrm{V}_{6} \mathrm{O}_{13}\right.$ : carbon black : EPDM (ethylene propylene diene copolymer) binder - 80: 15: 5 mass \%) was mixed for $1 \mathrm{~h}$ in a ceramic ball mill and then spread on aluminium foil using a wire bar. Cathodes with $2 \mathrm{~cm}$ diameter were punched out, transferred to a glove-box (Ar atmosphere; $\mathrm{O}_{2} / \mathrm{H}_{2} \mathrm{O}<5 \mathrm{ppm}$ ) and vacuum dried at $120{ }^{\circ} \mathrm{C}$ for $16 \mathrm{~h}$. Cells with a glass-fiber separator membrane and lithium foil as anode were assembled in the glove-box. The electrolyte was $1 \mathrm{M} \mathrm{LiBF}_{4}$ in a $2: 1$ by volume mixture of ethylene carbonate (EC) : diethyl carbonate (DEC). One cell was left uncycled to serve as reference. Another six cells were discharged to a potential corresponding to $1,2,3,4,5$ and 6 lithium ions. After equilibration, the cells were disassembled in the glovebox. In order to remove the salts and polymers, formed by side reactions, the cathodes were washed in dimethyl carbonate (DMC) for several hours. They were then flushed with DMC and dried under vacuum at room temperature overnight. The dried cathodes were cut into suitable pieces, mounted on sample holders and vacuum-packed in double plastic bags for transport. Further details about the $\mathrm{V}_{6} \mathrm{O}_{13}$ cathode sample preparation can be found in paper IX.

\section{Vanadium oxide nanotubes}

The vanadium oxide nanotubes were prepared as described by Krumeich et al [58]. A series of cycled cathodes was prepared by discharging batteries to different potentials $(3.0 \mathrm{~V}, 2.5 \mathrm{~V}, 2.0 \mathrm{~V}$ and $1.8 \mathrm{~V})$. Corresponding to the different cut-off potentials, the battery cathodes achieve different lithium content, i.e. one can prepare samples at different lithium doping levels. A more detailed description of the sample preparation, which is similar as the preparation of the $\mathrm{V}_{6} \mathrm{O}_{13}$ battery cathodes, can be found in paper $\mathrm{V}$. 



\section{Chapter 5}

\section{Results}

\subsection{Vanadium oxides}

This section deals with the investigation of the electronic structure of vanadium oxides $[5,59]$ with resonant soft X-ray emission spectroscopy (SXE). Vanadium oxides are found in a diversity of oxidation states with a broad variety of electronic, magnetic and structural material properties. In particular, many of them show metal-to-insulator transitions (MIT) [6], which are often accompanied by structural distortions of the $\mathrm{VO}_{6}$ octahedra or $\mathrm{VO}_{5}$ pyramids, the basic building blocks of their crystal structure. As common for $3 \mathrm{~d}$ transition metal oxides, the electronic behavior of different vanadium oxides is greatly dependent on their valence configuration, i.e. the oxidation state. Introducing an additional element into vanadium oxides of certain oxidation state, either by stochastic doping or stoichiometrically, leads to interesting changes in crystallographic and electronic structure. Electron or hole doping therefore significantly alters the electronic structure. For instance it is possible to change the transition temperatures for an occurring MIT continuously in a linear fashion by the amount of electron doping [60,61]. A well studied example in this connection is the electron doping of $\mathrm{V}_{2} \mathrm{O}_{3}$ with $\mathrm{Cr}$.

The following three subsections of the thesis discuss SXE spectra excited at $\mathrm{V}$ $2 \mathrm{p}$ and $\mathrm{O}$ 1s resonances of vanadium oxides with distinct oxidation state, $\mathrm{V}_{2} \mathrm{O}_{3}$ (paper II and VI) with a formal valence configuration of $\mathrm{V} 3 \mathrm{~d}^{2}\left(\mathrm{~V}^{3+}\right)$ and $\mathrm{VO}_{2}$ (paper II) with $\mathrm{V} 3 \mathrm{~d}^{1}\left(\mathrm{~V}^{4+}\right)$. The MIT of the $\mathrm{VO}_{2}$ system induced by temperature variation (paper XI) and the effect of doping in $\mathrm{Mo}_{x} \mathrm{~V}_{1-x} \mathrm{O}_{2}$ (paper VI) has been closely examined. The mixed valence compounds $\mathrm{V}_{6} \mathrm{O}_{13}$ (paper VIII), $\alpha$ '- $\mathrm{NaV}_{2} \mathrm{O}_{5}$ (paper II, VI and XV) and vanadium oxide nanotubes $\mathrm{Na}_{2} \mathrm{~V}_{3} \mathrm{O}_{7}$ (paper XII) are described in the last three subsections of this section.

\subsection{1 $\quad \mathrm{V}_{2} \mathrm{O}_{3}$}

$\mathrm{V}_{2} \mathrm{O}_{3}$ with a formal valence configuration of $\mathrm{V} 3 \mathrm{~d}^{2}$ undergoes a MIT at $160 \mathrm{~K}$ from a paramagnetic metal to an anti-ferromagnetic semiconductor $[62,63]$. The MIT is accompanied by a structural phase transition from trigonal to monoclinic crystal structure. Two-thirds of the distorted $\mathrm{O}_{6}$ octahedra, the basic building blocks of 

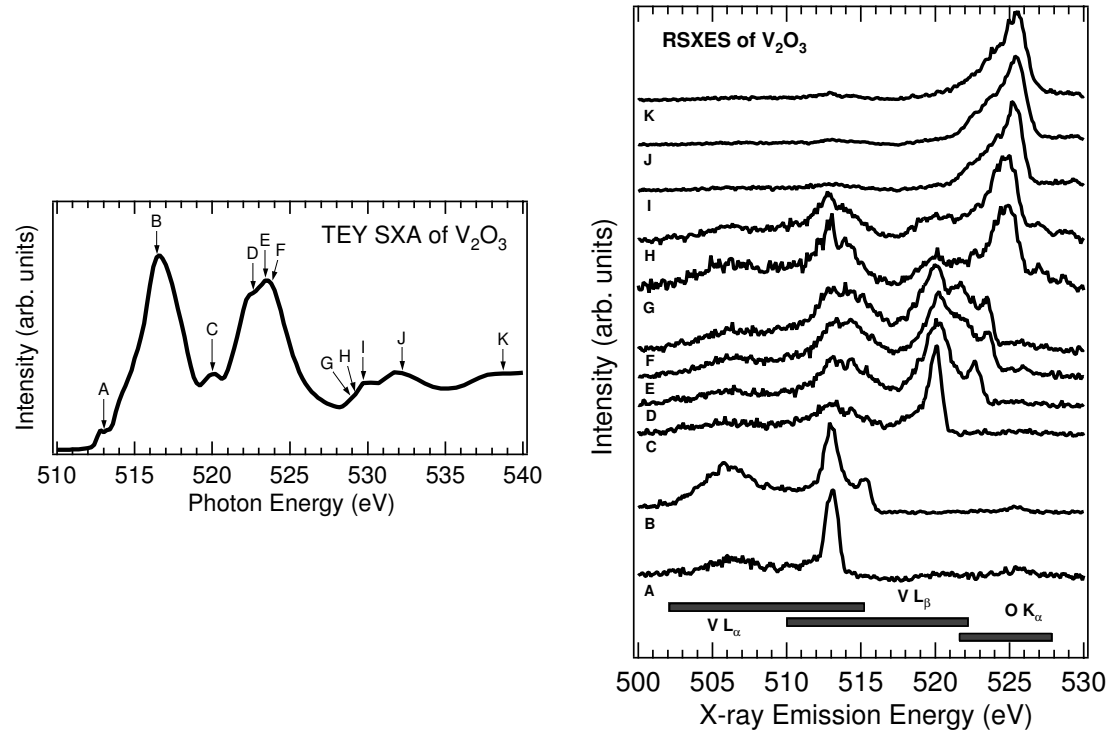

Figure 5.1: Resonant soft $\mathrm{X}$-ray emission spectra (right panel) of $\mathrm{V}_{2} \mathrm{O}_{3}$ excited at the energies A-K indicated in the V 2p - O 1s TEY SXA spectrum (left panel) above.

$\mathrm{V}_{2} \mathrm{O}_{3}$ crystals, are occupied with a $\mathrm{V}$ atom in the center. The prime mechanism for the MIT is generally accepted to be due to electron correlation effects and $\mathrm{V}_{2} \mathrm{O}_{3}$ is regarded a classical example of a Mott-Hubbard system [59,64,65].

The right panel of FIG. 5.1 displays a series of resonant SXE spectra of $\mathrm{V}_{2} \mathrm{O}_{3}$ excited at $\mathrm{V} 2 \mathrm{p}$ and $\mathrm{O} 1 \mathrm{~s}$ resonances at room temperature. All excitation energies are indicated by arrows (A-K) in a combined V 2p / O 1s SXA spectrum in the left panel. Changing the excitation energy results in a rich variation of spectral features, which can be seen in three energy regions. $\mathrm{V} \mathrm{L}_{\alpha}$ and $\mathrm{V} \mathrm{L}_{\beta}$ soft X-ray emission originate from $\mathrm{V} 3 \mathrm{~d} \rightarrow \mathrm{V} 2 \mathrm{p}_{3 / 2}$ respective $\mathrm{V} 2 \mathrm{p}_{1 / 2}$ transitions, which are centered around $512 \mathrm{eV}$ and $519 \mathrm{eV}$ in X-ray emission energy, respectively. The third emission band is the $\mathrm{O} \mathrm{K}_{\alpha}$ emission band situated at $524 \mathrm{eV}$, which is dominated by $\mathrm{O} 2 \mathrm{p} \rightarrow \mathrm{O}$ 1s electron transitions. The approximate extent of these three overlapping soft X-ray emission bands, is marked by bars at the energy scale of FIG. 5.1. Resonant excitation can be employed to restrict the spectral contributions in a defined manner, i.e. to selectively enhance certain band features by tuning the incident X-ray energy to an absorption threshold.

FIG. 5.2 (left panel) compares $\mathrm{V} \mathrm{L}_{\alpha}$-emission excited at the $\mathrm{V} 2 \mathrm{p}_{3 / 2}$ threshold (excitation energy $\mathrm{A}$ ) with $\mathrm{O} \mathrm{K}_{\alpha}$-emission (excitation energy $\mathrm{I}$ ). By aligning the spectra at the expected positions of the Fermi-level we see that both spectra consist of components that derive from $\mathrm{O} 2 \mathrm{p}$ partial density of states (PDOS) and V $3 \mathrm{~d}$ PDOS, i.e. that V $3 \mathrm{~d}$ and $\mathrm{O} 2 \mathrm{p}$ electron bands are strongly hybridized. In addition to spectral contributions that are due to the PDOS of $\mathrm{V} 3 \mathrm{~d}$ and $\mathrm{O} 2 \mathrm{p}$ character and are denoted as ordinary fluorescence, one also observes spectral signatures 

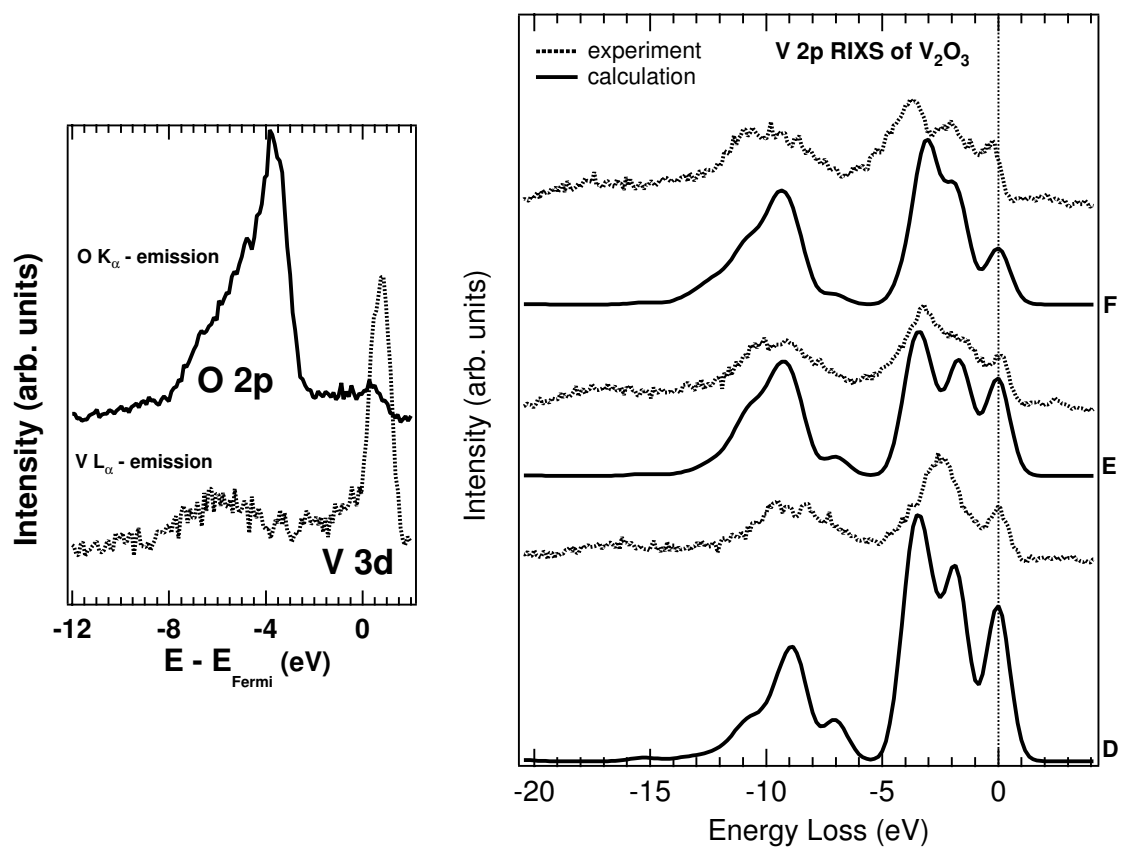

Figure 5.2: Left panel: Comparison of $\mathrm{O} \mathrm{K}_{\alpha}$ (excitation $\mathrm{I}$ ) and $\mathrm{V} \mathrm{L}_{\alpha}$ (excitation A) aligned on a binding energy scale. Both spectra of $\mathrm{V}_{2} \mathrm{O}_{3}$ consist of $\mathrm{O} 2 \mathrm{p}$ and $\mathrm{V}$ 3d components, that are strongly hybridized. Right panel: Comparison of resonant SXE spectra at the V $2 \mathrm{p}_{1 / 2}$ threshold compared to cluster model calculations.

that disperse with changing excitation energy, i.e. low energy excitations.

Spectral features with constant energy separation of ca. $1.8 \mathrm{eV}$ from the elastic peak, situated at the incident photon energy, arise in spectra D-H in FIG. 5.1. These energy loss features must be assigned to crystal field excitations, i.e. dd-excitations, which are prominent spectral signatures in resonant SXE spectra of late transition metal oxides. Band structure calculations cannot account for such excitations and a "more localized way" must be employed to describe the underlying processes by inelastic scattering theory using the Kramers-Heisenberg equation.

The right panel in FIG. 5.2 compares spectra D, E and F from FIG. 5.1 on an energy loss scale, placing the elastic peak at $0 \mathrm{eV}$, to theoretical spectra calculated within a cluster model (see Sec. 3.4). Experiment and theory agree reasonably regarding the energetic position of the dd-excitation at $-1.8 \mathrm{eV}$ energy loss between $\mathrm{d}$-orbitals of $\mathrm{t}_{2 g}$ and $\mathrm{e}_{g}$ symmetry. However, theory predicts also a second ddexcitation feature, which arises due to multiplet coupling, which is not observed in the experiment. This feature is probably obscured by the ordinary fluorescence contributions. Moreover, the cluster model calculations show a broad band of charge transfer (CT) excitations centered around ca. $9 \mathrm{eV}$ in energy loss. These $\mathrm{CT}$ excitations overlap significantly with the O $2 \mathrm{p}$ derived PDOS features from 
the ordinary fluorescence.

The fact that the relative spectral weights of the low energy excitations are much lower than the dominating ordinary fluorescence has been ascribed to the metallic character of $\mathrm{V}_{2} \mathrm{O}_{3}$ at room temperature (paper II and VI). For a metal, the intermediate states in RIXS should be relatively delocalized, resulting in relaxation processes, that lead to similar intermediate states as for normal soft X-ray emission. Therefore RIXS as a resonant (coherent) process should be suppressed in such cases.

However, preliminary studies on $\mathrm{V}_{2} \mathrm{O}_{3}$ samples cooled well below the MIT temperature of $160 \mathrm{~K}$ (not included in this thesis) did not reveal a change in the low energy excitation part of the spectra. This is in line with temperature dependent photoemission studies of $\mathrm{V}_{2} \mathrm{O}_{3}$, which only found the $\mathrm{V}$ 3d bandwidth to change slightly across the MIT [59]. Furthermore, relative spectral weight was transferred from bands of predominantly V 3 d character to bands of mostly O $2 \mathrm{p}$ character, i.e. the V 3d - O 2p hybridization was seen to change.

\subsection{2 $\quad \mathrm{VO}_{2}$ and $\mathrm{Mo}_{x} \mathrm{~V}_{1-x} \mathrm{O}_{2}$}

Vanadium dioxide $\mathrm{VO}_{2}$ with a formal valence configuration of $\mathrm{V} 3 \mathrm{~d}^{1}$ undergoes a MIT from a paramagnetic metal with tetragonal rutile structure to a nonmagnetic semiconductor with monoclinic structure manifested in a sudden conductivity drop of several orders of magnitude at $\mathrm{T}=340 \mathrm{~K}[66,67]$. Despite decades of studies the nature of these phases is not fully understood. Vanadium atoms are paired to dimers in the semiconducting phase. Therefore the main mechanism of the MIT has been ascribed to a crystallographic distortion and the resulting electronphonon interaction $[68,69]$ (Peierls-transition), opening a band gap by splitting the d-band [70]. Band structure calculations support this idea by explaining the crystallographic transition with the formation of a charge-density wave accompanied by lattice distortion and subsequent condensation of phonons [68]. However, the energy gain from the crystallographic distortion alone might not be enough to open up the gap and thus electron correlation effects [59,71] (Mott-transition) may play an important role as well.

The resonant SXE spectra excited at V 2p and O 1s resonances presented in paper II show that the electronic structure at room temperature appears to be mostly band-like with a low degree electron correlation. This conclusion had earlier been drawn by Kurmaev et al. [72] in a combined experimental and theoretical study of the electronic structure of $\mathrm{VO}_{2}$.

Doping of $\mathrm{VO}_{2}$ with electron donors (e.g. with $\mathrm{W}$ and $\mathrm{Mo}$ ) results in a linear decrease of the MIT temperatures proportional to the dopant concentration. Metallic monoclinic $\mathrm{MoO}_{2}[73]$ is isostructural to the low temperature semiconducting phase of $\mathrm{VO}_{2}$. By doping $\mathrm{VO}_{2}$ with Mo the MIT temperature for the $\mathrm{Mo}_{x} \mathrm{~V}_{1-x} \mathrm{O}_{2}$ compound system is decreased $[60,61] . \mathrm{Mo}_{x} \mathrm{~V}_{1-x} \mathrm{O}_{2}$ possess industrial relevance, since thin films of $\mathrm{Mo}_{x} \mathrm{~V}_{1-x} \mathrm{O}_{2}$ are used as thermochromic window coatings [74]. By doping $\mathrm{VO}_{2}$ with $\mathrm{x}=8 \%$ and $18 \%$ Mo the MIT temperature of undoped $\mathrm{VO}_{2}, \mathrm{~T}_{M I T}(0 \% \mathrm{Mo})=340 \mathrm{~K}$, is lowered to $\mathrm{T}_{M I T}(8 \% \mathrm{Mo})=225 \mathrm{~K}$ and $\mathrm{T}_{M I T}(18 \% \mathrm{Mo})=130 \mathrm{~K}$, respectively. 
SXE spectra excited at V L thresholds are associated mainly with V 3d PDOS. In paper VI, we investigated how spectral weight increases with the Mo content $\mathrm{x}$ and this spectral development is related to a filling of the $\mathrm{V} 3 \mathrm{~d}$ band due to a corresponding increase of electron doping. Similar effects will be discussed later on in the studies of Li-ion insertion into vanadium oxide battery cathodes (see Sec. 5.2). The V L SXE spectra display nearly a rigid shift of the spectral weight originating predominantly from $\mathrm{O} 2 \mathrm{p}-\mathrm{V}$ 3d hybridized PDOS upon doping $\mathrm{VO}_{2}$ with Mo. This spectral behavior is interpreted as a consequence of the $\mathrm{V} 3 \mathrm{~d}$ bandfilling and a quasi-rigid band behavior. The spectral evolution of the O K-emission remarkably reveals that the band width for $\mathrm{x}=18 \%$ is much narrower than for $\mathrm{x}$ $=0$ and $8 \%$. A possible explanation could be a change in the degree of $\mathrm{O} 2 \mathrm{p}-\mathrm{V}$ $3 \mathrm{~d}$ hybridization induced by the Mo doping.

\subsubsection{MIT in $\mathrm{VO}_{2}$}

Paper XI presents a combined experimental and theoretical investigation of electronic structure changes arising in $\mathrm{VO}_{2}$ across the MIT, or more precisely across the metal-to-semiconductor transition (MST). SXA recorded in the total fluorescence yield mode and resonant SXE excited at V 2p and O 1s resonances, were employed to monitor the bulk electronic structure of the valence band.

The spectral evolution of the V 2p and O 1s SXA spectra across the MST is similar as discovered in earlier investigations [75-77]. Importantly, the unoccupied $\pi^{*}$ band (i.e. the band of $\mathrm{t}_{2 g}$ derived states) is lifted only $0.2-0.3 \mathrm{eV}$ above the Fermi-level in the semiconducting phase. This is a significantly smaller rise than observed earlier from ultraviolet reflection by Shin et al. [59], who anticipated a shift of $0.5 \mathrm{eV}$ away from the Fermi-energy.

The results from SXE at V $2 \mathrm{p}$ and $\mathrm{O}$ 1s edges are in line with earlier photoemission investigations, i.e. the semiconducting phase showed a sharpening of the occupied V 3d bands compared the metallic phase [78,79]. For comparison V L and $\mathrm{O}$ K x-ray emission spectra have been derived from band structure calculations employing the local density approximation of the density functional theory. Experimental and theoretical spectra are in good qualitative agreement regarding relative spectral development across the MST.

All V L SXE spectra displayed in FIG. 5.3 have been normalized to maximum intensity of the band situated around $510 \mathrm{eV}$ in X-ray emission energy, which can mainly be ascribed to $\mathrm{O} 2 \mathrm{p}$ states hybridized with V $3 \mathrm{~d}$ states. For excitations at the $\mathrm{V} 2 \mathrm{p}_{3 / 2}$ threshold, the SXE spectra A and B possess a band around $514 \mathrm{eV}$, originating from $\mathrm{V} 3 \mathrm{~d}$ states, that receives increased spectral weight in the metallic phase. A corresponding band around $521 \mathrm{eV}$ for the $\mathrm{V} 2 \mathrm{p}_{1 / 2}$ excitation energies $\mathrm{C}$ and $\mathrm{D}$ becomes even more enhanced when heating the sample from the room temperature semiconducting phase to the metallic phase at $100^{\circ} \mathrm{C}$. Relative spectral weight is shifted from bands of $\mathrm{V} 3 \mathrm{~d}-\mathrm{O} 2 \mathrm{p}$ character at high binding energies to the band of nearly pure V $3 \mathrm{~d}$ character at low binding energy, when heating the sample to $100^{\circ} \mathrm{C}$. This relative spectral development reflects the decreasing $\mathrm{V} 3 \mathrm{~d}$ - O 2p hybridization in the metallic phase of $\mathrm{VO}_{2}$. 

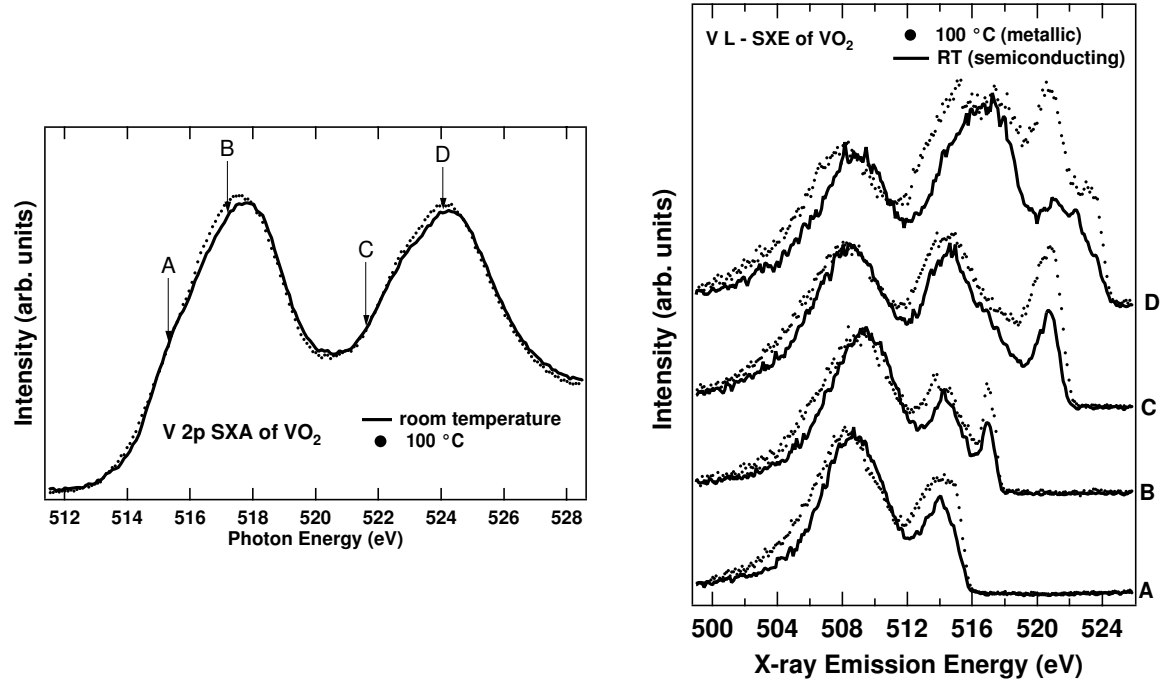

Figure 5.3: Resonant soft $\mathrm{X}$-ray emission spectra of $\mathrm{VO}_{2}$ above $(373 \mathrm{~K})$ and below (room temperature) the MIT temperature $\mathrm{T}=340 \mathrm{~K}$ excited at $\mathrm{V} 2 \mathrm{p}_{3 / 2}$ (A and $\mathrm{B}$ ) and $\mathrm{V} 2 \mathrm{p}_{1 / 2}(\mathrm{C}$ and $\mathrm{D})$ resonances.

\subsection{4 $\quad \mathrm{V}_{6} \mathrm{O}_{13}$}

$\mathrm{V}_{6} \mathrm{O}_{13}$ is a monoclinic mixed-valence compound (electron configuration: $4 \mathrm{~V}^{4+}$ $+2 \mathrm{~V}^{5+}$ ) that undergoes a MST at $150 \mathrm{~K}$ that is accompanied by a structural distortion from the paramagnetic metal phase with $\mathrm{C} 2 / \mathrm{m}$ symmetry [80,81] to the paramagnetic semiconducting phase with C2 symmetry [81] or P21/a symmetry [82]. Antiferromagnetic order occurs below ca. 55K. The basic building blocks of the crystal are distorted $\mathrm{VO}_{6}$-octahedra $[80,81]$ which form two types of sheets parallel to the a-b plane. One zigzag $\mathrm{VO}_{6}$ chain running along the b-axis is monovalent $\left(\mathrm{V}^{4+}\right)$ and a second chain is of mixed valency (alternating $\mathrm{V}^{4+}$ and $\left.\mathrm{V}^{5+}\right)[80,81]$.

Ordinary photoemission spectroscopy (PES) [59] and angular resolved photoemission spectroscopy (ARPES) [83] have been used to study the valence band electronic structure of $\mathrm{V}_{6} \mathrm{O}_{13}$. Particular attention has been paid to the MST of $\mathrm{V}_{6} \mathrm{O}_{13}$. Small changes in the valence band width have been observed by PES, however, the trends of ordinary PES and ARPES appear to be of opposite nature, i.e. band narrowing is observed upon cooling in ordinary PES and band broadening in ARPES. Both experiments, however, seem to show a gap opening at the Fermi level, characteristic for the semiconducting state.

$\mathrm{V}_{6} \mathrm{O}_{13}$ receives strong attention as an active electrochemical component in cathodes of model systems for novel compact rechargeable Li-batteries where Li is inserted into the cathode material. Paper IX and X, summarized in the next section, deal with the soft X-ray spectroscopic investigation of Li-ion insertion into $\mathrm{V}_{6} \mathrm{O}_{13}$ containing battery cathodes. 


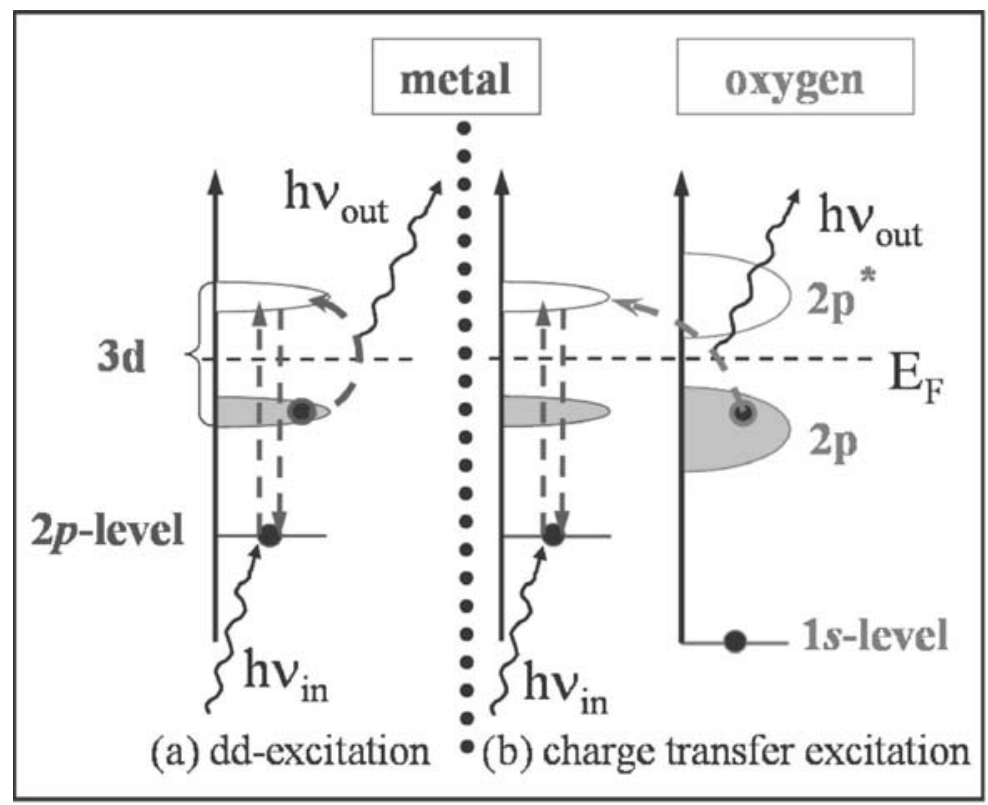

Figure 5.4: Crystal field and charge transfer excitations.

In paper VIII, the electronic structure of $\mathrm{V}_{6} \mathrm{O}_{13}$ single crystals is investigated by means of SXA and SXE spectroscopy. This paper gives a combined experimental and theoretical analysis of the electronic structure of $\mathrm{V}_{6} \mathrm{O}_{13}$ focusing on the role and interplay of band-like and localized excitations close to the ground state. SXA and SXE spectroscopy at the $\mathrm{V}$ L-edges is employed to monitor changes in the electronic structure across the MST of $\mathrm{V}_{6} \mathrm{O}_{13}$.

In the left panel of FIG. 5.5 we plotted resonant SXE spectra A-I excited at $\mathrm{V}$ L thresholds against an energy loss scale relative to the elastic peaks, in order to facilitate an easier identification of so called excitonic features. FIG. 5.4 schematically characterizes the types of low-energy local excitations, that are responsible for these characteristic excitonic features. The prominent structure at an energy loss of approximately $-1.6 \mathrm{eV}$ is assigned to a local dd-excitation within the crystal field split $3 \mathrm{~d}$ multiplet $\left(\mathrm{t}_{2 g} \rightarrow \mathrm{e}_{g}\right.$ ) (see the left hand side of FIG. 5.4). Obviously, the $\mathrm{VO}_{6}$ cluster model calculation presented in the right panel of FIG. 5.5 gives a good account for this dd-excitation peak.

A broad band of charge transfer (CT) excitations $\mathrm{O} 2 \mathrm{p} \rightarrow \mathrm{V} 3 \mathrm{~d}$ (see the right hand side of FIG. 5.4) is situated around an energy loss of $-7 \mathrm{eV}$ in FIG. 5.5 (left panel). However, the detailed spectral shape variation of the theoretical CT-band (see FIG. 5.5, right panel) is not observed in the experimental spectra. Partially, this is probably due to significant ordinary fluorescence contributions originating from the $\mathrm{V} 3 \mathrm{~d}$ PDOS of nearly pure and $\mathrm{V} 3 \mathrm{~d}-\mathrm{O} 2 \mathrm{p}$ hybridized character, similarly as in $\mathrm{VO}_{2}$ and $\mathrm{V}_{2} \mathrm{O}_{3}$, which can be accounted for by DFT band structure calculations. Moreover, a more sophisticated modelling of the lowenergy excitations would also require the use of multi-site clusters [24] which is 

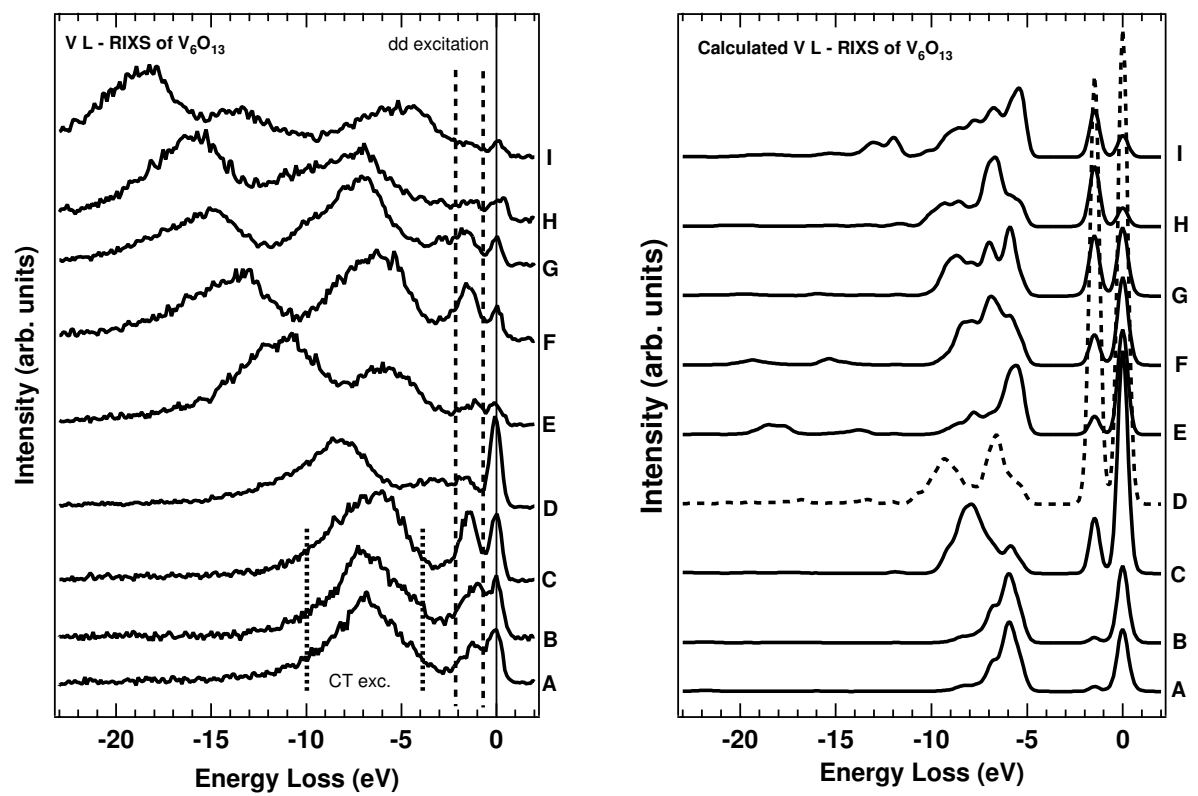

Figure 5.5: Experimental and calculated RIXS spectra of $\mathrm{V}_{6} \mathrm{O}_{13}$ excited at V Ledge energies.

yet computationally too time consuming.

In the study of the MST of $\mathrm{V}_{6} \mathrm{O}_{13}$, small changes are observed in the $\mathrm{V} \mathrm{L}_{\alpha}$-SXE spectra recorded at $295 \mathrm{~K}$ and $120 \mathrm{~K}$, that reflect the valence band structure. These trends are corroborated by temperature dependent band structure calculations. It is discovered that the MST of $\mathrm{V}_{6} \mathrm{O}_{13}$ is accompanied by changes in the valence band structure in a similar fashion as observed in PES [59,83], i.e. a small shift of the main V3d-band towards the Fermi energy in the semiconducting phase. This study demonstrates that SXE can give valuable complementary spectroscopic information on the subject of MST in vanadates and the use of brighter X-ray sources would be favorable for a more extensive investigation of the MST with SXE spectroscopy.

\subsection{5 $\quad \alpha^{\prime}-\mathrm{NaV}_{2} \mathrm{O}_{5}$}

$\alpha^{\prime}-\mathrm{NaV}_{2} \mathrm{O}_{5}$ possesses orthorhombic crystal structure at room temperature and is centro-symmetric (Pmmn) with only one distinct $\mathrm{V}$ ion in a mixed valence state (average valency $\mathrm{V}^{+4.5}$ ) [84]. The spins are carried by $\mathrm{V}-\mathrm{O}-\mathrm{V}$ rungs of molecular orbitals, the basic building blocks of the electronic structure. At $34 \mathrm{~K}$, insulating $\alpha^{\prime}-\mathrm{NaV}_{2} \mathrm{O}_{5}$ undergoes a low-temperature phase transition, which has been debated to be of charge ordering or spin-Peierls-like type $[85,86]$. The room temperature insulating state of $\alpha^{\prime}-\mathrm{NaV}_{2} \mathrm{O}_{5}$ has been theoretically investigated extensively by band structure calculations within the framework of the local density approxi- 


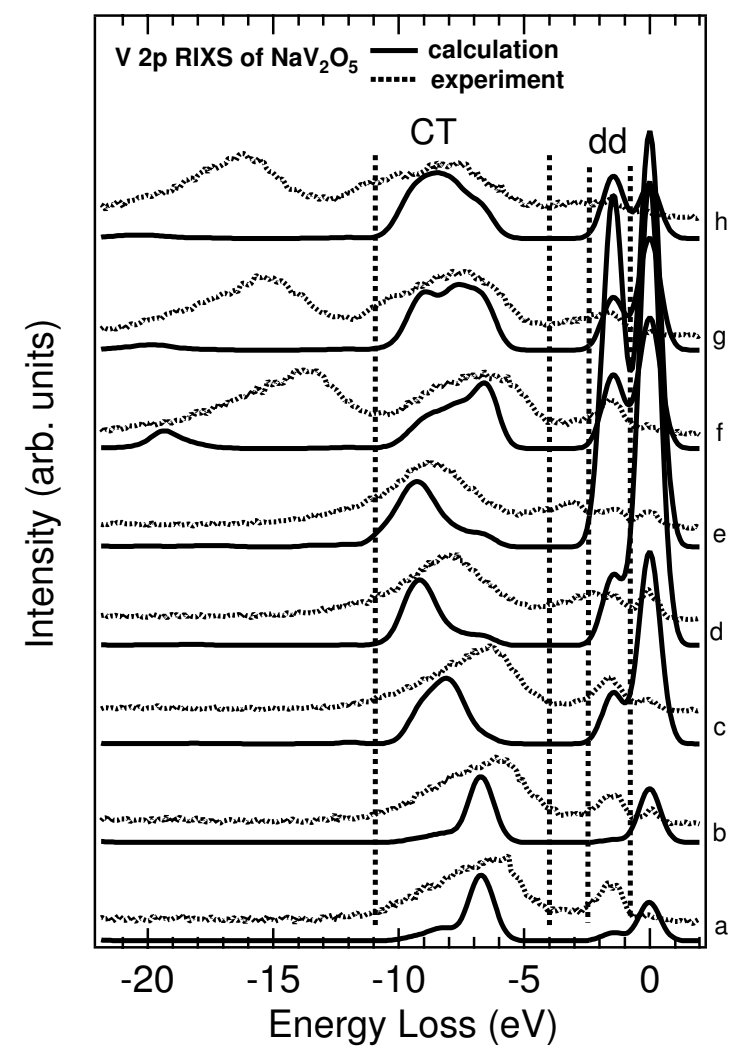

Figure 5.6: Experimental V2p-RIXS spectra of $\alpha^{\prime}-\mathrm{NaV}_{2} \mathrm{O}_{5}$ (dotted lines) and the corresponding theoretical cluster model calculations (solid lines).

mation (LDA) [87-90] and by LDA band structure calculations including on-site Coulomb correlation energy U (LDA + U) [88-91]. By including electron correlations accounted for by the Hubbard $U$ the insulating character of $\alpha^{\prime}-\mathrm{NaV}_{2} \mathrm{O}_{5}$ is explained. This visualizes the strongly correlated nature of its electronic structure. As a key feature, the $\mathrm{V} 3 \mathrm{~d}_{x y}$ band is split by the correlation effects into an upper and lower Hubbard band opening a gap around the Fermi level [89].

There is some confusion about, wether this correlation gap can be observed by RIXS at V 2p resonances [92]. Major parts of the papers II, VI and VII deal with a combined experimental and theoretical RIXS investigation of $\alpha^{\prime}-\mathrm{NaV}_{2} \mathrm{O}_{5}$. Paper $\mathrm{XV}$ comments on the origin of the low-energy excitation part of the V L-RIXS spectra and gives clear evidence, why probably the correlation induced band gap in $\alpha^{\prime}-\mathrm{NaV}_{2} \mathrm{O}_{5}$ is impossible to study with V L-RIXS.

In FIG. 5.6 experimental RIXS spectra (dotted lines) excited at the V 2p resonances (a)-(h) are plotted against an energy loss scale relative to the elastic peaks and are compared to performed cluster model calculations (solid lines). This visualizes, similarly as for the investigation of $\mathrm{V}_{6} \mathrm{O}_{13}$ described in the previous sub- 
section, that cluster model calculations give a good account for the dd-excitation peak, marked by dashed lines at a constant energy loss of $-1.6 \mathrm{eV}$ in the experimental spectra. The similarity between the resonating behavior and energy positions of this peak in (metallic) $\mathrm{V}_{6} \mathrm{O}_{13}$ and in (insulating) $\alpha^{\prime}-\mathrm{NaV}_{2} \mathrm{O}_{5}$ strongly suggests that it has the same origin in both compounds, namely a local dd-excitation, i.e. a crystal field excitation on a $\mathrm{d}^{1}$-ion.

A recent study by Zhang et al. [92] revealed the same energy loss feature in $\alpha^{\prime}$ $\mathrm{NaV}_{2} \mathrm{O}_{5}$ at a similar value of $1.56 \pm 0.05 \mathrm{eV}$. They attributed this salient spectral feature to a dd-excitation from the lower occupied Hubbard band V $3 \mathrm{~d}_{x y}$ to the upper unoccupied Hubbard band $\mathrm{V} 3 \mathrm{~d}_{x y}^{\star}$. However, LSDA+U band structure calculations [89] revealed a much smaller d-d transition gap of $0.6 \mathrm{eV}$. In paper $\mathrm{XV}$ it is pointed out that RIXS involves core levels, and therefore only the matrix elements of local transitions have significant strength. The correlation-induced gap that is responsible for the insulating behavior of $\alpha^{\prime}-\mathrm{NaV}_{2} \mathrm{O}_{5}$ can, on the other hand, only be probed by inter-rung transitions. However, transitions between these states are believed to give weak spectral signatures in V L-edge RIXS, because the $2 \mathrm{p}$ core hole has very small overlap with d-orbitals on neighboring rungs. Therefore, it seems at least very difficult to separate spectral contributions from transitions across the correlation gap from the dominating dd-excitation in RIXS investigations with the present spectral sensitivities.

FIG. 5.7 shows a resonant O K-emission spectrum excited at $531.7 \mathrm{eV}$, i.e. at the V 3d-hybridized low-energy shoulder of the oxygen O1s-absorption (see inset). A RIXS peak, resonating in a narrow excitation energy interval, with an energy loss of about $1.2 \mathrm{eV}$ for the bonding-antibonding gap on a rung $\mathrm{d}_{x y}$ - $\mathrm{d}_{x y}^{*}$ (not for the correlation gap) is consistent with optical spectroscopy [87, 93, 94]. Hybridization of the $\mathrm{O} 2 \mathrm{p}$-orbitals with $\mathrm{V} 3 \mathrm{~d}$-orbitals is, therefore, probably the key that can reveal nominally non-local transitions of electrons involving more than one $\mathrm{V}$-site. However, on-rung transitions are believed to be much stronger than inter-rung transitions and it seems also difficult to probe the correlation gap with $\mathrm{O}$ K-edge RIXS.

The spectral behavior of CT excitations and ordinary fluorescence contributions in the V 2p-RIXS spectra is quite similar as the one observed for $\mathrm{V}_{6} \mathrm{O}_{13}$. The good agreement of the excitonic contributions in the experimental V $2 p$ RIXS spectra with the cluster model calculations in paper VI corroborates the assumption of the highly correlated nature of $\alpha^{\prime}-\mathrm{NaV}_{2} \mathrm{O}_{5}$. Furthermore, the ratio of excitonic to ordinary fluorescence contributions gives a phenomenological measure of the localization in the intermediate core-hole state. From the dominance of the excitonic RIXS contributions in spectrum (a)-(c) can be concluded that the states in the threshold region are strongly localized and indicative for a highly correlated material.

\subsection{6 $\quad \mathrm{Na}_{2} \mathrm{~V}_{3} \mathrm{O}_{7}$ nanotubes}

The crystal structure of trigonal $\mathrm{Na}_{2} \mathrm{~V}_{3} \mathrm{O}_{7}$ (space group P31c) is built up by $\mathrm{VO}_{5}$ square pyramids connected at the edges and corners to form nanotubes with an inner diameter of $5 \AA$ [55]. $\mathrm{Na}^{+}$ions, situated inside and around the nanotubes, result in vanadium ions of the formal oxidation state $\mathrm{V}^{4+}$. Nine square pyramids 


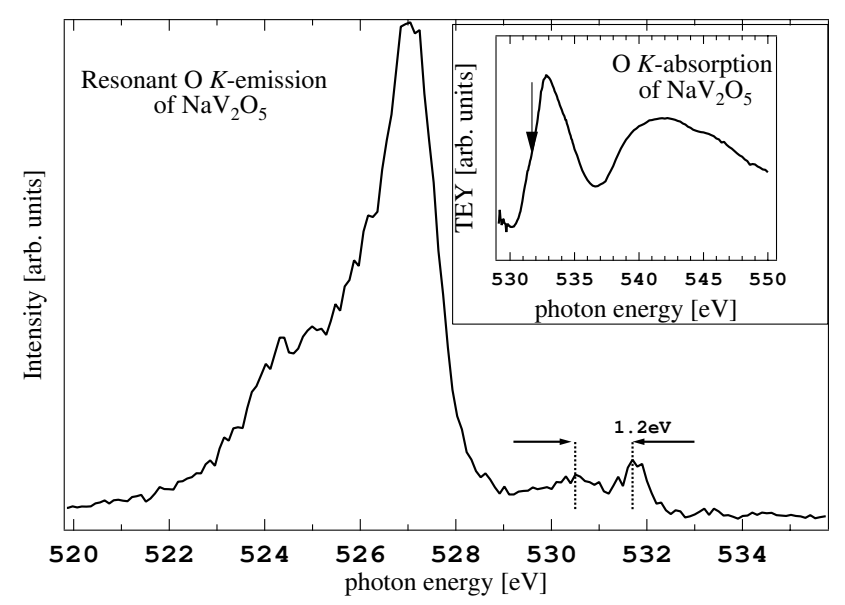

Figure 5.7: $\mathrm{O}$ K - RIXS of $\alpha^{\prime}-\mathrm{NaV}_{2} \mathrm{O}_{5}$. Inset: Total electron yield (TEY) SXA spectrum across the $\mathrm{O}$ K-edge.

constitute rings forming the nanotubes by stacking the rings along the c-axis and two neighboring rings build a unit cell. All vanadium atoms are situated in the center of the pyramids at three inequivalent vanadium sites $V_{1}, V_{2}$ and $V_{3}$. Oxygen atoms occur at seven inequivalent sites, where $\mathrm{O}_{1}, \mathrm{O}_{2}$ and $\mathrm{O}_{3}$ are the apices of the vanadium square pyramids, which point out from the nanotubes and correspond to the $\mathrm{V}_{1}, \mathrm{~V}_{2}$ and $\mathrm{V}_{3}$ sites, respectively. The remaining oxygen sites $\mathrm{O}_{4}, \mathrm{O}_{5}, \mathrm{O}_{6}$ and $\mathrm{O}_{7}$ are situated at the basal positions of the $\mathrm{VO}_{5}$ pyramids [55].

In paper XII, we present a combined theoretical and experimental study of the electronic structure of $\mathrm{Na}_{2} \mathrm{~V}_{3} \mathrm{O}_{7}$ nanotubes. SXA and resonant SXE spectra have been recorded across the V 2p- and the O 1s-resonances. The occurrence of both, localized excitations (i.e. crystal field and charge transfer excitations) and band-like features, in the resonant V L-SXE spectra reveal a dual nature of the electronic structure of $\mathrm{Na}_{2} \mathrm{~V}_{3} \mathrm{O}_{7}$. Localized low-energy excitations are due to the local character of the valence band electronic structure and can be simulated by a cluster model calculation. DFT band structure calculations account for the partially non-local character, which gives rise to band-like spectral features.

Both kinds of spectrum in $\mathrm{Na}_{2} \mathrm{~V}_{3} \mathrm{O}_{7}$ are more similar to the spectra of the mixed valence compounds $\mathrm{V}_{6} \mathrm{O}_{13}$ described in paper VIII (possessing formally $4 \mathrm{~V}^{4+}$ and $2 \mathrm{~V}^{5+}$ sites) and $\mathrm{NaV}_{2} \mathrm{O}_{5}$ described in paper VI (possessing sites with fractional oxidation state $\mathrm{V}^{4.5+}$ ), and quite different from the $\mathrm{V}^{4+}$-material $\mathrm{VO}_{2}$ [59]. The V L-SXE spectra of $\mathrm{VO}_{2}$ do not show large contributions of local character (see paper II and XI). Therefore, we conclude that the oxidation state of $\mathrm{Na}_{2} \mathrm{~V}_{3} \mathrm{O}_{7}$ probably departs from a purely monovalent $\mathrm{V}^{4+}$ system and shows also a partial $\mathrm{V}^{5+}$-character.

In FIG. 5.8(a), we present calculated oxygen site-projected O K-SXE spectra displayed in the order suggested by the corresponding O 1s SXA spectra. As expected, the spectral shape and energetic position of the apical spectra $\mathrm{O}_{1}, \mathrm{O}_{2}$ and 

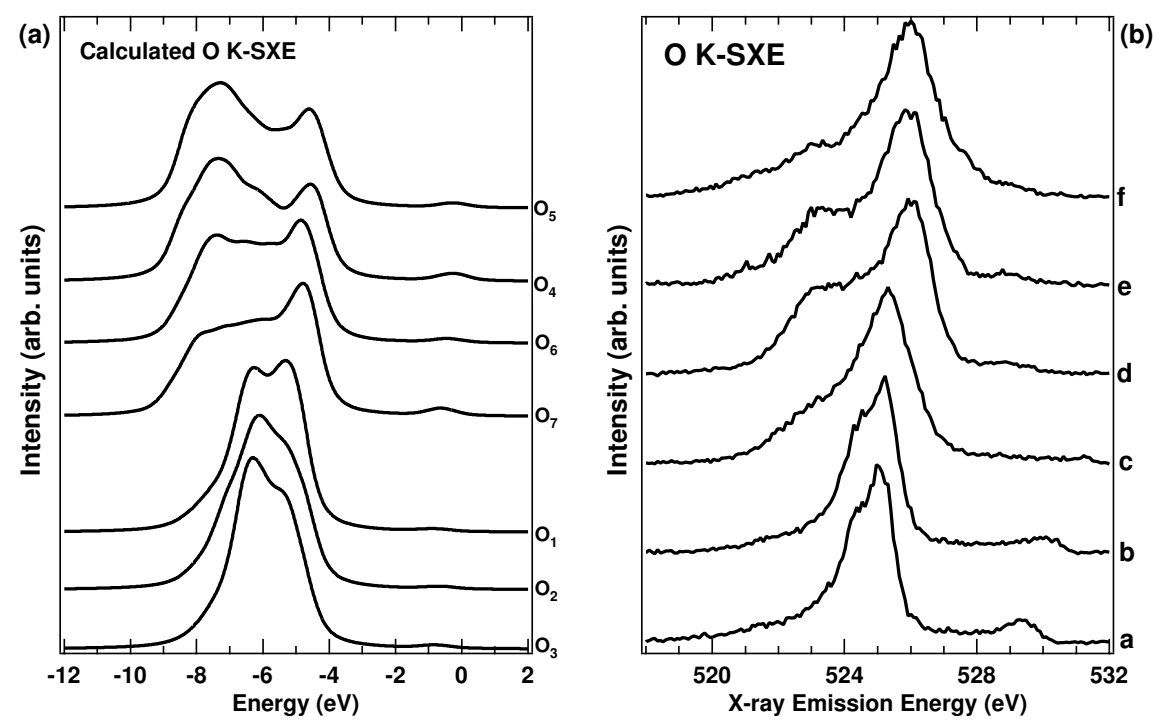

Figure 5.8: (a): Theoretical oxygen site-projected $\mathrm{O}$ K-SXE spectra of $\mathrm{Na}_{2} \mathrm{~V}_{3} \mathrm{O}_{7}$ derived from a DFT band structure calculation. (b): Experimental O K-SXE spectra excited at the $\mathrm{O} 1 \mathrm{~s}$ resonances a - $\mathrm{f}$.

$\mathrm{O}_{3}$ is significantly different from the basal spectra $\mathrm{O}_{4}, \mathrm{O}_{5}, \mathrm{O}_{6}$ and $\mathrm{O}_{7}$. For the excitations " $c$ " - " $\mathrm{f}$ " in the experimental O K-SXE spectra in FIG. 5.8(b), two peaks are observed, a broad low energy peak centered at $523 \mathrm{eV}$ and a more structured high energy peak with a center of gravity around $526 \mathrm{eV}$. The threshold excited $\mathrm{O}$ K-emission spectra "a" and "b" show a relatively sharp emission band around 525 $\mathrm{eV}$. A direct comparison between theoretical and experimental O K-SXE spectra in FIG. 5.8 (a) and (b) evidences that spectra "a" and "b" predominantly represent occupied O 2p PDOS of the apical oxygen sites, whereas for the higher excitation energies "c" - "f" basal oxygen sites have dominant contributions.

\subsection{Lithium insertion into vanadium oxide cath- odes}

Li-ion batteries [95] are presently satisfying the continuously growing demand for efficient compact power sources for modern portable electronic equipment. A lithium-ion battery comprises a lithium containing transition metal oxide (TMO) cathode, a Li-ion conducting polymer or organic solvent as electrolyte, a Li-salt, and often a graphite anode [95]. During the charging of a Li-ion battery using a LiTMO cathode [95] (e.g. $\mathrm{LiCoO}_{2}$ ), lithium ions are extracted from the cathode and inserted into the anode (graphite). The reverse reaction takes place on discharge. If the cathode material does not contain lithium as- synthesized, a lithium foil is generally used as anode [95]. FIG. 5.9 depicts a schematic representation of the 


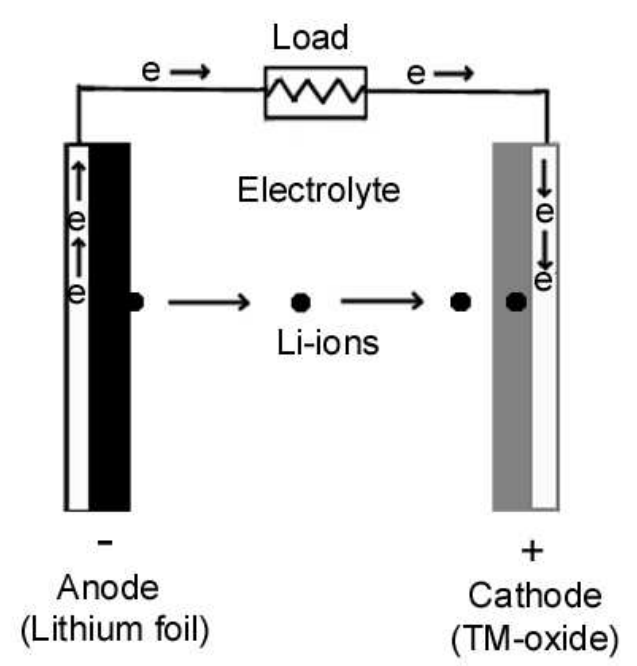

Figure 5.9: Schematic drawing of a Li-transition metal oxide battery [96].

discharge process in a Li-battery using Li foil as anode and a TMO without Li as cathode.

\subsection{1 $\quad \mathrm{V}_{6} \mathrm{O}_{13}$ battery cathodes}

An interesting cathode material with very high capacity (ca. $310 \mathrm{mAh} / \mathrm{g}$ ), which has gained widespread attention, is $\mathrm{V}_{6} \mathrm{O}_{13}[3,4]$. The electrochemical lithiation process of $\mathrm{V}_{6} \mathrm{O}_{13}$ is characterized by a stepwise transition between phases of $\mathrm{Li}_{x} \mathrm{~V}_{6} \mathrm{O}_{13}$ with $\mathrm{x}=0,2 / 3,1, \ldots, 6$ resulting in a "staircase" shape of the electrode potential as a function of lithium content (see paper IX for details). In such a curve plateaus correspond to two-phase regions and vertical potential drops to singlephases of discrete stoichiometry [97]. The crystal structure of $\mathrm{V}_{6} \mathrm{O}_{13}$, depicted in FIG. 5.10, comprises distorted $\mathrm{VO}_{6}$ octahedra with shared edges and corners [80]. $\mathrm{VO}_{6}$ octahedra form a single and a double layer, connected via shared corners.

Electrochemically lithiated $\mathrm{Li}_{x} \mathrm{~V}_{6} \mathrm{O}_{13}(\mathrm{x}=0,1, \ldots, 6)$ has been investigated by studying battery cathodes of $\mathrm{V}_{6} \mathrm{O}_{13}$ discharged to a given voltage using a Li-metal anode. The $\mathrm{Li}_{x} \mathrm{~V}_{6} \mathrm{O}_{13}$ samples so-prepared were studied ex-situ by SXA and SXE spectroscopies at room temperature. SXA and SXE spectroscopies were used to investigate the occupied and unoccupied electronic states of both vanadium and oxygen close to the Fermi level. Paper IX reports on oxidation state changes during the lithiation process investigated by SXA. Li-insertion is accompanied by electron transfer to vanadium sites. Paper $\mathrm{X}$ elaborates how the valence band of $\mathrm{V}_{6} \mathrm{O}_{13}$ is modified in a nearly rigid band like manner and compares SXE spectra exited at the $\mathrm{V} \mathrm{L}_{3}$-edge with DFT electronic band structure calculations. The interpretation of all spectral features in the SXE and SXA follows the study of 


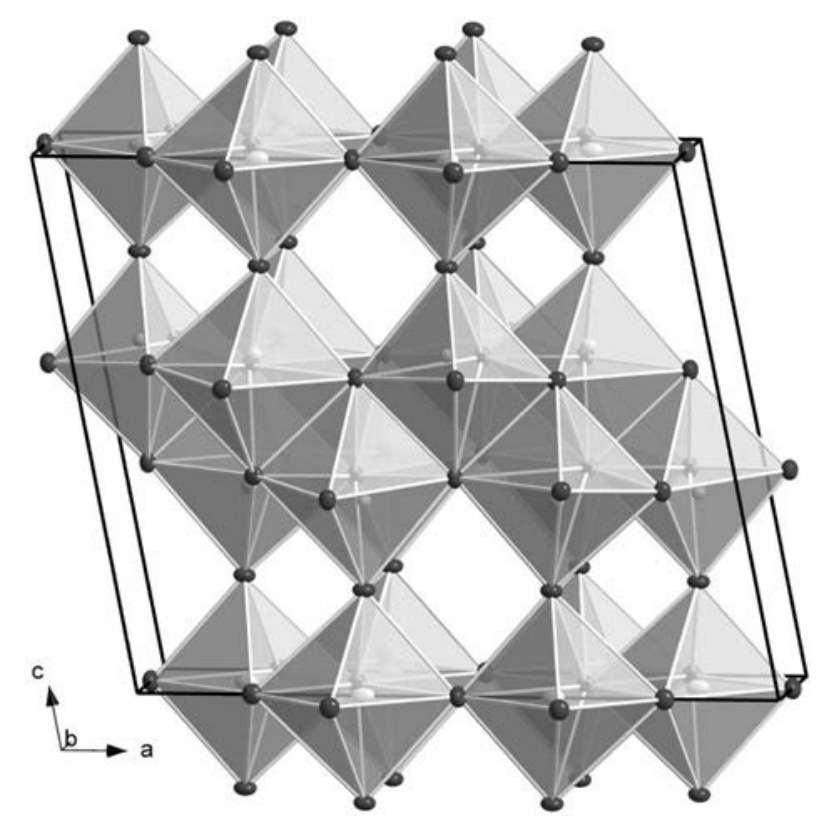

Figure 5.10: The crystal structure of $\mathrm{V}_{6} \mathrm{O}_{13}$ comprising $\mathrm{VO}_{6}$ octahedra as building blocks. Black and white spheres represent oxygen and vanadium atoms, respectively.

single crystalline $\mathrm{V}_{6} \mathrm{O}_{13}$ presented in paper VIII.

FIG. 5.11(a) shows SXA spectra of $\mathrm{Li}_{x} \mathrm{~V}_{6} \mathrm{O}_{13}$ cathodes with Li-contents $\mathrm{x}=$ $0,1, \ldots, 6$. The spectrum for the non-lithiated reference cathode $(\mathrm{x}=0)$ closely resembles the SXA spectra of single-crystal $\mathrm{V}_{6} \mathrm{O}_{13}$ samples (see paper VIII). V L-absorption spectra for $\mathrm{VO}_{2}\left(\mathrm{~V}^{4+}\right)$ and $\mathrm{V}_{2} \mathrm{O}_{3}\left(\mathrm{~V}^{3+}\right)$ have been inserted into the graph to fit into the spectral evolution of the $\mathrm{Li}_{x} \mathrm{~V}_{6} \mathrm{O}_{13}$ spectral series. From this one can conclude that lithiation of $\mathrm{V}_{6} \mathrm{O}_{13}$ produces mostly $\mathrm{V}^{4+}$-ions $\left(\mathrm{VO}_{2}\right)$ at $2<\mathrm{x}<3$ and mostly $\mathrm{V}^{3+}$-ions $\left(\mathrm{V}_{2} \mathrm{O}_{3}\right)$ at $4<\mathrm{x}<5$. Furthermore, the spectra for $\mathrm{x}=6$ can be interpreted as containing even some $\mathrm{V}^{2+}$-ions as occurring in VO.

From an electrical conductivity study of chemically lithiated nonstoichiometric $\mathrm{V}_{6} \mathrm{O}_{13}$, Chaklanabish and Maiti [98] concluded that the reduction of $\mathrm{V}^{4+}$ to $\mathrm{V}^{3+}$ preceeds that of $\mathrm{V}^{5+}$ to $\mathrm{V}^{4+}$ as the Li content increases. By contrast, our present study of the electrochemical Li insertion into stoichiometric $\mathrm{V}_{6} \mathrm{O}_{13}$ supports the findings of Stallworth et al. [99] that the reduction process goes from $\mathrm{V}^{5+}$ to $\mathrm{V}^{4+}$ (between $\mathrm{x}=0$ and $\mathrm{x}=2$ ) and then to $\mathrm{V}^{3+}$.

$\mathrm{O}$ K-SXA spectra for the $\mathrm{Li}_{x} \mathrm{~V}_{6} \mathrm{O}_{13}$ series are shown in FIG. 5.11(b). The first peak (dashed line at about $529.5 \mathrm{eV}$ ) is associated with empty $\mathrm{O} 2 \mathrm{p}$-orbitals that are strongly hybridized with V 3d-orbitals. Decreasing intensity of this peak with increasing $\mathrm{x}$ is an indication that the inserted $\mathrm{Li}$ atoms donate electrons, which almost completely fill the empty V $3 \mathrm{~d}-\mathrm{O} 2 \mathrm{p}$ hybridized states at $\mathrm{x}=6$. These $\mathrm{O}$ K-SXA spectra are as well compared with those from the vanadate model systems $\mathrm{V}_{2} \mathrm{O}_{5}, \mathrm{~V}_{6} \mathrm{O}_{13}, \mathrm{VO}_{2}$, and $\mathrm{V}_{2} \mathrm{O}_{3}$. At low Li-content, the $\mathrm{O}$ K-SXA resembles more the case of $\mathrm{V}_{2} \mathrm{O}_{5}$, which contains only $\mathrm{V}^{5+}$-ions; only for $\mathrm{x}=2$ do we see peak ratios 


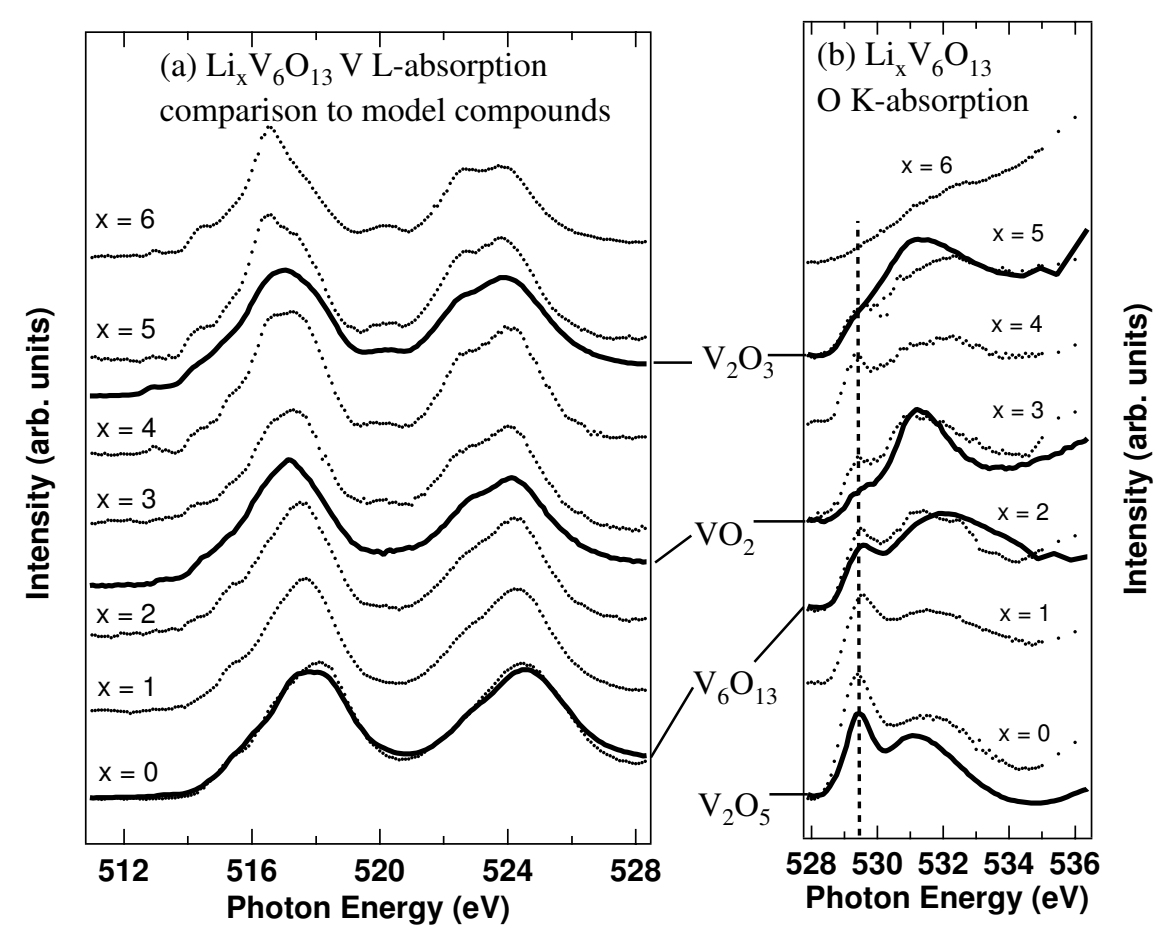

Figure 5.11: (a) Dotted curves: V L-absorption spectra of $\mathrm{Li}_{x} \mathrm{~V}_{6} \mathrm{O}_{13}$ battery cathodes in total electron yield mode. Solid lines: V L-absorption spectra of $\mathrm{V}_{6} \mathrm{O}_{13}$, $\mathrm{VO}_{2}$, and $\mathrm{V}_{2} \mathrm{O}_{3}$ (from bottom to top). (b) Dotted curves: O K-absorption spectra of $\mathrm{Li}_{x} \mathrm{~V}_{6} \mathrm{O}_{13}$ battery cathodes recorded in total electron yield mode. Solid lines: $\mathrm{O}$ $\mathrm{K}$-absorption spectra of $\mathrm{V}_{2} \mathrm{O}_{5}, \mathrm{~V}_{6} \mathrm{O}_{13}, \mathrm{VO}_{2}$, and $\mathrm{V}_{2} \mathrm{O}_{3}$ (from bottom to top).

which resemble those for $\mathrm{V}_{6} \mathrm{O}_{13}$. For higher Li-content, the situation is similar again to the result from $\mathrm{V}$ L-SXA. An explanation for the difference between the results for $\mathrm{V} \mathrm{L}$ - and O K-SXA may lie in the difference in the absorption coefficients, which is larger for $\mathrm{O}$ K-absorption, meaning that it can be more sensitive to material closer to the surface and possibly influenced by contamination.

FIG. 5.12 shows a comparison between (a) V L -SXE spectra and (b) results of DFT band structure calculations. Both, experiment and theory show that the bands are filled at the Fermi-level in a rather rigid manner, i.e. the top of the valence band gains weight and the bottom of the valence band is shifted down in energy. Furthermore, the experimental result shows a strong decrease in the spectral weight of the V3d-O2p hybridized states as a result of a decrease of the overlap between the $\mathrm{V} 3 \mathrm{~d}$ and the $\mathrm{O} 2 \mathrm{p}$-orbitals for increasing Li-content. X-ray diffraction measurements for $\mathrm{Li}_{x} \mathrm{~V}_{6} \mathrm{O}_{13}$ (for Li-contents up to $\mathrm{x}=3$ ) [97, 100-102] show a linear dependence on the effective charge at the $\mathrm{V}$-sites. This is consistent with our present results, which display a linear dependence of the integrated peak intensities of the band associated with nearly pure V 3d states (see FIG. 5.12(c)), 

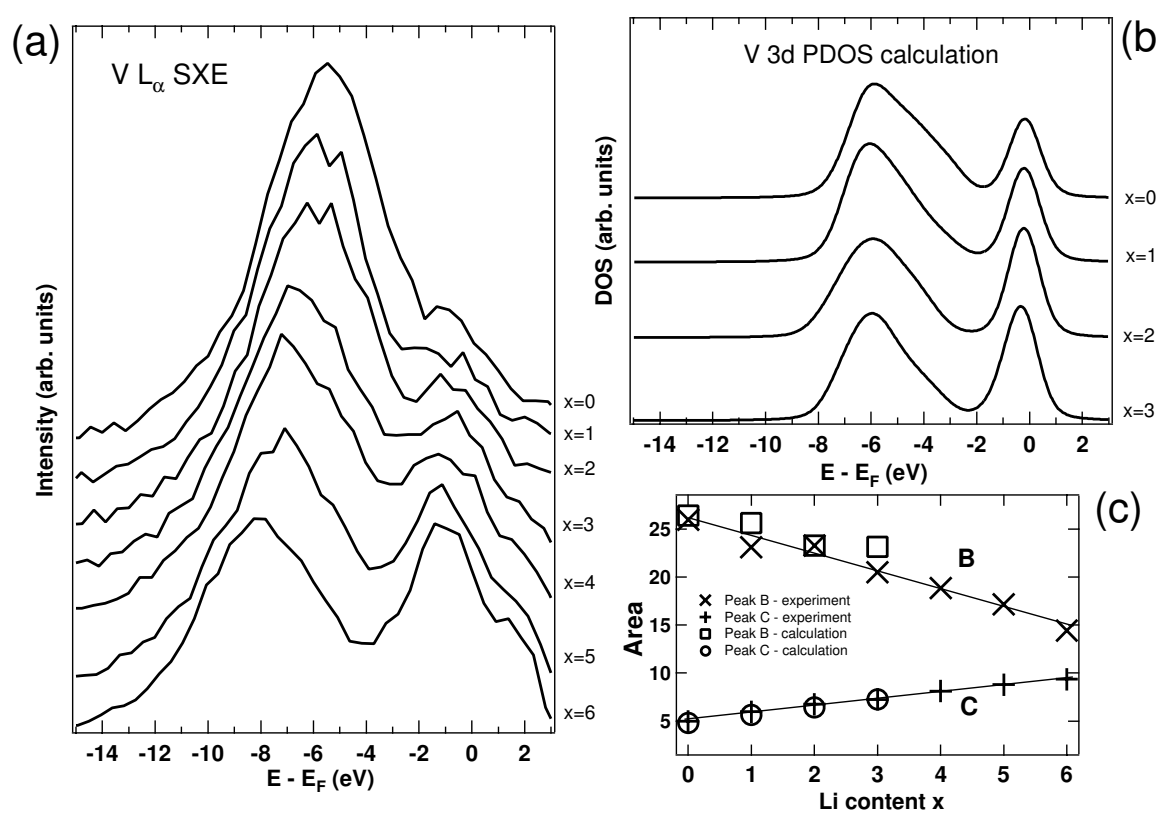

Figure 5.12: Comparison of the $\mathrm{V} \mathrm{L}_{\alpha}$ SXE spectra (a) with spectra derived from $\mathrm{V}$ $3 \mathrm{~d}$ PDOS calculations (b). Integrated peak intensities of the nearly pure V3d peak $\mathrm{C}$ and peak B deriving from V3d-O2p hybridized states are shown for both, SXE experiment and PDOS calculations, in (c).

extending over the entire lithiation range.

\subsubsection{Cathodes of vanadium oxide nanotubes}

Paper IV and V study Na-containing vanadium oxide (Na-VOx) nanotubes used as cathodes for rechargeable Li-batteries. This nanotubular material consists of scroll-like layers of vanadium oxide separated by structure-directing agents, in this case $\mathrm{Na}^{+}$ions [58], and has recently demonstrated to be a well-functioning cathode material for rechargeable Li-batteries $[103,104]$. Li-insertion into the NaVOx nanotubes has been studied by SXA (paper IV and V), SXE (paper V) and XPS (paper IV) in order to investigate the effects of the lithiation on the electronic structure.

A series of cycled cathodes were prepared by discharging the batteries to different potentials $(3.0 \mathrm{~V}, 2.5 \mathrm{~V}, 2.0 \mathrm{~V}$ and $1.8 \mathrm{~V})$ resulting in different content of lithium. Similarly as in the case of $\mathrm{Li}_{x} \mathrm{~V}_{6} \mathrm{O}_{13}$, the gradual changes in the spectra are attributed to electron transfer into unoccupied V 3d states as Li-ions are inserted into the material. As lithium ions are inserted into the Na-VOx nanotubes, the V 2p peak weight shifts towards lower energies. This effect can be seen in both the SXA and XPS results (paper IV). Both, SXA and SXE (paper V) measurements at $\mathrm{V} 2$ p-edges point to a partial reduction to $\mathrm{V}^{3+}$ at potentials below 2.0 
V. The excitation energy dependence of the V L-SXE spectra indicates a charge separation in the material into coexisting $\mathrm{d}^{2}, \mathrm{~d}^{1}$ and $\mathrm{d}^{0}$ vanadium sites at high degree of lithiation.

\subsection{Insulating Cuprates}

The last section of the summarized results of this thesis presents a SXA and resonant SXE spectroscopy study on the electronic structure of insulating copper oxides. Whereas vanadium oxides, discussed in the previous two sections, show moderate degree of electron correlation together with a mostly band-like character, copper oxides are regarded as strongly correlated systems. The bonding between copper and oxygen atoms gives rise to strong hybridization effects and therefore even the itinerant oxygen 2 p-bands reflect the correlation effects of the strongly correlated $\mathrm{Cu} 3 \mathrm{~d}$ electrons [105]. Many theories of the electronic structure of high- $\mathrm{T}_{c}$ superconducting cuprates assume that coupling between the $\mathrm{Cu} 3 \mathrm{~d}$ and O $2 \mathrm{p}$ orbitals plays an important role for the transition to the superconducting state [105].

Most cuprates are constituted of $\mathrm{Cu}^{2+}$-ions, i.e the copper atom has transferred two electrons to oxygen orbitals, and therefore the copper d-orbitals are generally occupied with nine 3 d-electrons, where one $3 \mathrm{~d}$-orbital is singly occupied and the other four are fully occupied. Insulating cuprates are the parent "undoped" compounds of high- $\mathrm{T}_{c}$ superconducting cuprates and are ideal model system for spectroscopic studies of the $\mathrm{Cu}-\mathrm{O}$ octahedra, the basic building blocks of the crystal structure of most copper oxides. This section demonstrates how complementary information of the electronic structure in a variety of insulating cuprates can be gained employing resonant SXE spectroscopy excited a $\mathrm{O} 1 \mathrm{~s}, \mathrm{Cu}$ $2 \mathrm{p}, \mathrm{Cu} 3 \mathrm{~s}$ and $\mathrm{Cu} 3 \mathrm{p}$ absorption-edges. The focus of these investigations lies at the $\mathrm{O} 1 \mathrm{~s}$ and $\mathrm{Cu} 3 \mathrm{p}$-edges.

\subsubsection{Resonant SXE spectroscopy at O 1s resonances}

\section{$\mathrm{CuGeO}_{3}$}

$\mathrm{CuGeO}_{3}$ is an orthorhombic antiferromagnetic insulator (with an optical absorption band gap of $3.7 \mathrm{eV}$ ) with the lattice parameters $a=4.81 \AA, b=8.47 \AA$, $c=2.94 \AA$ at room temperature . The one-dimensional edge-sharing $\mathrm{CuO}_{4}$ chains of $\mathrm{CuGeO}_{3}$ are coupled along its crystallographic $c$-axis, in contrast to high $\mathrm{T}_{c}$ cuprates that have vertex-sharing chains.

Paper I addresses the valence band electronic structure of this unusual inorganic spin-Peierls material $\left(\mathrm{T}_{S P}=14 \mathrm{~K}\right)$ [106] investigated by resonant $\mathrm{SXE}$ at $\mathrm{O}$ 1s resonances. It is shown, that $\mathrm{O} 1 \mathrm{~s}-\mathrm{SXE}$ reflects oxygen-specific valence states with both bandlike as well as localized (excitonic) character. The localized states are observed as RIXS features that resonate only at the pre-edge peak of the $\mathrm{O}$ $1 s$-SXA spectrum, associated with the upper Hubbard band consisting of $\mathrm{O} 2 \mathrm{p}$ - $\mathrm{Cu} 3 \mathrm{~d}$ hybridized states of largely localized character [105, 107]. Main band ordinary fluorescence features derived from the symmetry-projected $2 p$-DOS at 


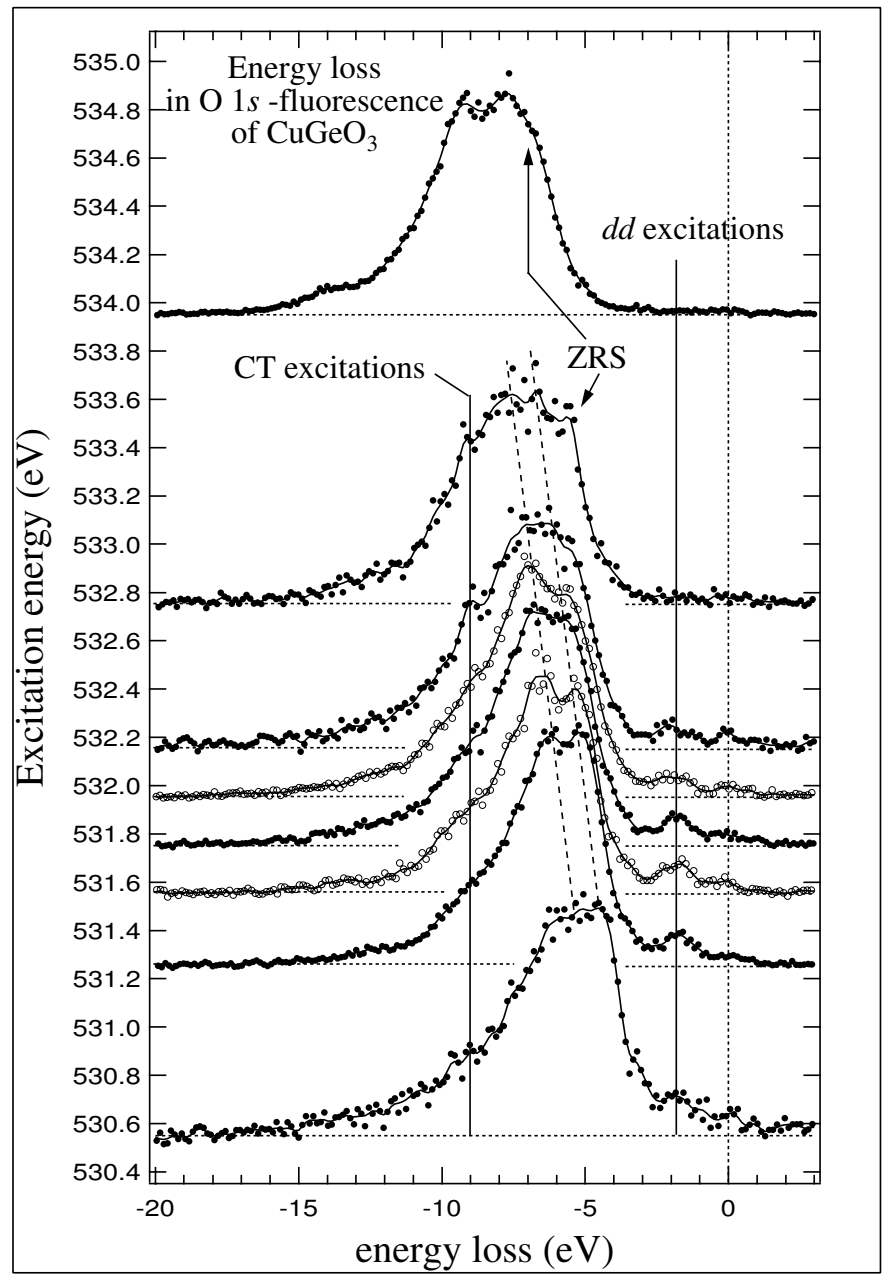

Figure 5.13: $\mathrm{CuGeO}_{3} \mathrm{O} \mathrm{K}-\mathrm{SXE}$ spectra excited at various energies ( $e \| a$ geometry) plotted against an energy loss scale. The raw spectra are shown as circles and the superimposed solid lines are corresponding three-point binomially smoothed spectra.

different oxygen sites arise from the delocalized oxygen valence band states. Inplane excited SXE spectra evidence the occurrence of a so called Zhang-Rice singlet [108] displayed as a high-energy shoulder of the O K-emission band.

FIG. 5.13 displays SXE spectra resonantly excited at various energies close to the two first $\mathrm{O}$ 1s absorption maxima. The spectra are plotted against an energy loss scale so that RIXS features appear at constant loss energies (solid vertical lines) and ordinary fluorescence disperses apparently to larger energy losses (dashed lines) with increasing excitation energy. We associate the peak with the lowest energy loss (about $1.85 \mathrm{eV}$ ) with $d d$-excitations, resonating when tuning across the pre-edge peak $(531.7 \mathrm{eV})$, slightly lower than the $d d$-excitations found 
in $\mathrm{Cu} 2 p$-RIXS (2.02 eV).

The transitions for Cu $p$-edge RIXS are predominantly $d^{9} \rightarrow \underline{c} d^{10} \rightarrow d^{9^{*}}(d d$ excitations) and $d^{9} \rightarrow \underline{c} d^{10} \rightarrow d^{10} \underline{L}$ (CT-excitations), where $\underline{c}$ stands for a $\mathrm{Cu}$ $p$-core hole and $\underline{\mathrm{L}}$ for a ligand hole. Generally, the ground state $\mathrm{Cu} 3 d$-hole has $x^{2}-y^{2}$-symmetry and the excitation energy of this hole to orbitals of different symmetry (denoted as $d^{9^{*}}$ ) is measured as the energy loss of the $d d$-excitation. For $\mathrm{Cu}^{2+}$-systems the oxygen $1 s$-absorption prepeak is associated with transitions to the upper Hubbard band and has been explained [107] by mixing of ligand-hole states $\left(d^{10} \underline{L}\right)$ into its ground state. The ligand hole is filled in the absorption process leaving a $d^{10}$ intermediate state in the RIXS process which subsequently decays into a mixture of $d^{10} \underline{L}$ - and $d^{9 *}$-states, the same final states as in $\mathrm{Cu} p$-edge RIXS. Moreover, a shoulder at an energy loss of about $9 \mathrm{eV}$, tentatively marked with a vertical line at $9.0 \mathrm{eV}$ in Fig. 5.13, originates probably from CT excitations, but overlaps strongly with ordinary fluorescence.

The strong energy dependent spectral shape of the main band is partly due to weight shifts of SXE from inequivalent oxygen sites. The crystal structure of $\mathrm{CuGeO}_{3}$ reveals [109] that there are two inequivalent oxygen sites: in-plane oxygen and apical oxygen. For excitation energies around the first absorption maximum, only O K-SXE from the in-plane oxygen is emitted since it is below the threshold for apical oxygen emission. At high-energy excitation O K-SXE from both sites are superposed with different weights and energy offsets. Polarization dependence of the O K-SXE spectra is also analyzed in paper I in great detail and reflects the difference in spectral weight from $\mathrm{O} 2 p_{x, y^{-}}$and $2 p_{z}$-orbitals.

A shoulder on the low-energy-loss flank develops at higher excitation energies and has no correspondence in the O $2 p$-DOS. We interpret this as a signature for a Zhang-Rice singlet (ZRS), i.e. double hole states of a mixture of $d^{9} L, d^{8}$, and $d^{10} \underline{L}^{2}$, where the excited electron leaves the atom. Such a spectral feature should, therefore, only occur when tuning to excitation energies that promote the electron into a delocalized oxygen orbital. The ZRS shows the same polarization dependence as the in-plane oxygen part of the bandlike states. Recently, theoretical model calculations using the Anderson impurity approach $[110,111]$ showed in detail how ZRS excitations of different character could contribute to the O 1s-RIXS signal in resonantly excited O K-emission.

\section{Comparison of O 1s-RIXS in $\mathrm{CuGeO}_{3}, \mathrm{CuO}, \mathrm{La}_{2} \mathrm{CuO}_{4}$, and $\mathrm{SrCuO}_{2}$}

Parts of paper III and VII compare O 1s-RIXS spectra of $\mathrm{CuGeO}_{3}$ to the related cuprates $\mathrm{CuO}, \mathrm{La}_{2} \mathrm{CuO}_{4}$, and $\mathrm{SrCuO}_{2}$. FIG. 5.14 (left panel) displays O 1s-SXE spectra resonantly excited at the upper Hubbard band prepeak of these cuprates. The sharp feature found between the elastic peak and the main band in each spectrum (marked with vertical bars) displays Raman-like behavior, i.e. it disperses linearly with excitation energy. Ab initio calculations by C. de Graaf and R. Broer [112] predicted the values for dd-excitations to d-orbitals of different symmetry for a number of cuprate compounds. The right panel of FIG. 5.14 displays a comparison between the dd-excitation energy values from the current O 1s-RIXS data and the ab-initio values from reference [112]. 


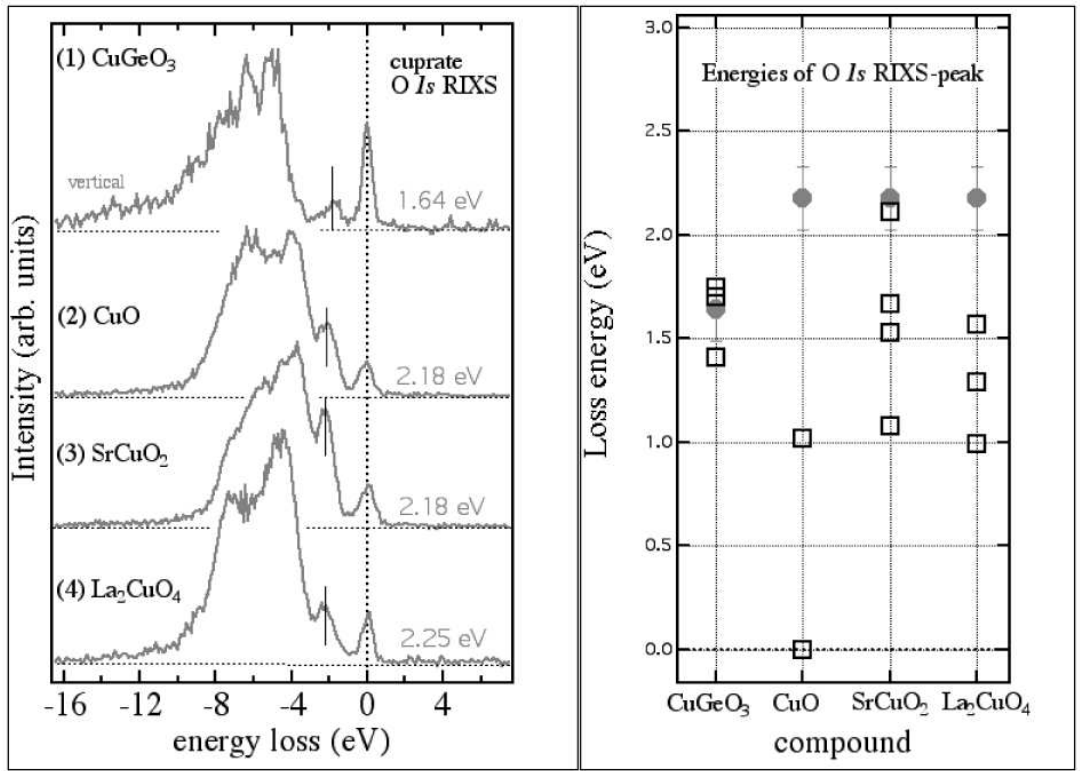

Figure 5.14: Left panel : O 1s-RIXS for various compounds excited at the prepeak on an energy transfer scale. Right panel: Peak values of the first energy loss structure. Circles show the data taken by O 1s-RIXS, open squares show theoretical predictions of dd-excitations from literature [112] (note that theoretical values of the Sr-cuprate were calculated for the similar $\mathrm{Sr}_{2} \mathrm{CuO}_{3}$.

Theory agrees best with the present experimental results for $\mathrm{CuGeO}_{3}$. For $\mathrm{CuO}, \mathrm{La}_{2} \mathrm{CuO}_{4}$, and $\mathrm{SrCuO}_{2}$ we observe significantly higher loss energies than the values for dd-excitations calculated by de Graaf and Broer. Therefore the question arises of whether other transitions, such as ZRS transitions, might contribute to the observed O1s-RIXS peaks of $\mathrm{CuO}, \mathrm{La}_{2} \mathrm{CuO}_{4}$, and $\mathrm{SrCuO}_{2}$. On the other hand, also recent $\mathrm{Cu} 3 \mathrm{p}$-RIXS experiments (see next subsection) show similar ddexcitation energies as the ones from $\mathrm{O} 1 \mathrm{~s}$-RIXS. Very recent $\mathrm{Cu} 2 \mathrm{p}$ RIXS studies by Ghiringhelli et al. [113] reported a dd-excitation peak around -1.5 eV for $\mathrm{La}_{2} \mathrm{CuO}_{4}$, which agrees qualitatively well with the calculations by de Graaf and Broer. The same authors compared their results for the dd-excitation in $\mathrm{CuO}$ of $-1.7 \mathrm{eV}$ with simple crystal field model calculations, which revealed $-1.38 \mathrm{eV}$ (for $\mathrm{b}_{2 g}$ symmetry), $-1.73 \mathrm{eV}\left(\mathrm{a}_{1 g}\right)$ and $-1.99 \mathrm{eV}\left(\mathrm{e}_{g}\right)$ [113]. Thus, the observed differences between results for the dd-excitations determined with RIXS at different absorption edges might be explainable by a dependence of the transition sensitivity to different final state symmetries depending on the experimental method used.

\section{Polarization dependent O 1s-RIXS of $\mathrm{SrCuO}_{2}$}

In paper XIV polarized resonant soft X-ray emission spectroscopy at O 1s-resonances could be used to probe the oxygen related valence band electronic structure of the 
quasi one-dimensional cuprate material $\mathrm{SrCuO}_{2}$. Employing the excitation energy dependence of the SXE spectra allows for the identification of spectral signatures of localized and delocalized character. Effects of electron localization are reflected by dd-excitations occurring when the excitation energy is tuned to the upper Hubbard band, which consists of $\mathrm{Cu} 3 \mathrm{~d}-\mathrm{O} 2 \mathrm{p}$ hybridized states of excitonic-like character. Bandlike O 2 p-states give rise to a main band of ordinary fluorescence, which is dominating when the intermediate core excited state is of rather delocalized character. Excitation energy and polarization dependence gives siteand symmetry projected PDOS information. Furthermore, strong indications for Zhang-Rice singlet states are found for excitation energies higher than electron transitions to the upper Hubbard band, i.e for intermediate core excited states of rather delocalized character.

\subsubsection{Resonant inelastic soft X-ray scattering at $\mathrm{Cu} 3 \mathrm{p}$ and $\mathrm{Cu} 3$ s resonances}

In paper III and VII we present $\mathrm{Cu} 3 \mathrm{p}$ - and $\mathrm{Cu} 3$ s-RIXS spectra of $\mathrm{CuGeO}_{3}$. The $\mathrm{Cu} 3$ s-experiments were performed at the circularly polarized beamline $4.2 \mathrm{R}$ at Elletra, Trieste. $\mathrm{Cu}$ 3p-RIXS measurements have been performed at beamline 10.0.1 at the Advanced Light Source of the Lawrence Berkeley National Laboratory, USA. FIG. 5.15 illustrates the main advantage of performing RIXS experiments at very shallow core levels, namely that the achievable instrumental resolution is substantially improved. The Cu 2p-RIXS spectrum shows dd- and charge transferexcitations, while the $\mathrm{Cu} 3 \mathrm{p}$-RIXS spectrum only shows the dd-excitation. This example shows that the absolute resolution in a RIXS experiment is roughly ten times better at the $\mathrm{Cu} 3$ p-resonance than at the $\mathrm{Cu} 2 \mathrm{p}$-resonance, while the relative resolution is comparable at about $1 / 1000$. This is an essential improvement in view of the small energy separations of for instance $\mathrm{Cu}$ dd-excitations to different orbital symmetries (in the order of $0.1 \mathrm{eV}$ ).

The $\mathrm{Cu} 3$ s-RIXS investigation in paper III and VII shows low-energy loss structures at around $1.7 \mathrm{eV}$, i.e. at similar energy values as the dd-excitations in the $\mathrm{Cu} 3 \mathrm{p}$-RIXS study. If they are due to dd-excitations, these RIXS features are extraordinary and must be indirectly induced. For p-edge RIXS at 3d-transition metal L- and M-edges dipole selection rules allow a direct transition to and from the 3d-states. For s-edge RIXS a Cu 3s-excited electron must fill a p-like empty state. This means that this intermediate state must have a sizeable coupling or hybridization with d-like states.

The complexity of the RIXS spectra near the $\mathrm{Cu}$ 3s-threshold (see paper III and VII) at higher energy losses finds its explanation in the coincidental overlap with Ge 3p-RIXS. The high-energy structures are effective valence band to conduction band transitions of Ge-derived states. Their relatively large intensity in the RIXS spectrum is somewhat surprising for being transitions between delocalized states. This effect can be used to obtain a high-resolution convolution between the occupied and unoccupied density of states.

Paper XIII reports polarization and energy dependent high-resolution RIXS experiments at the $\mathrm{Cu} 3 p$ absorption resonances of $\mathrm{CuGeO}_{3}, \mathrm{SrCuO}_{2}, \mathrm{CuO}$ and $\mathrm{La}_{2} \mathrm{CuO}_{4}$. For $\mathrm{CuGeO}_{3}$, we observe three close-lying excitations that agree well 


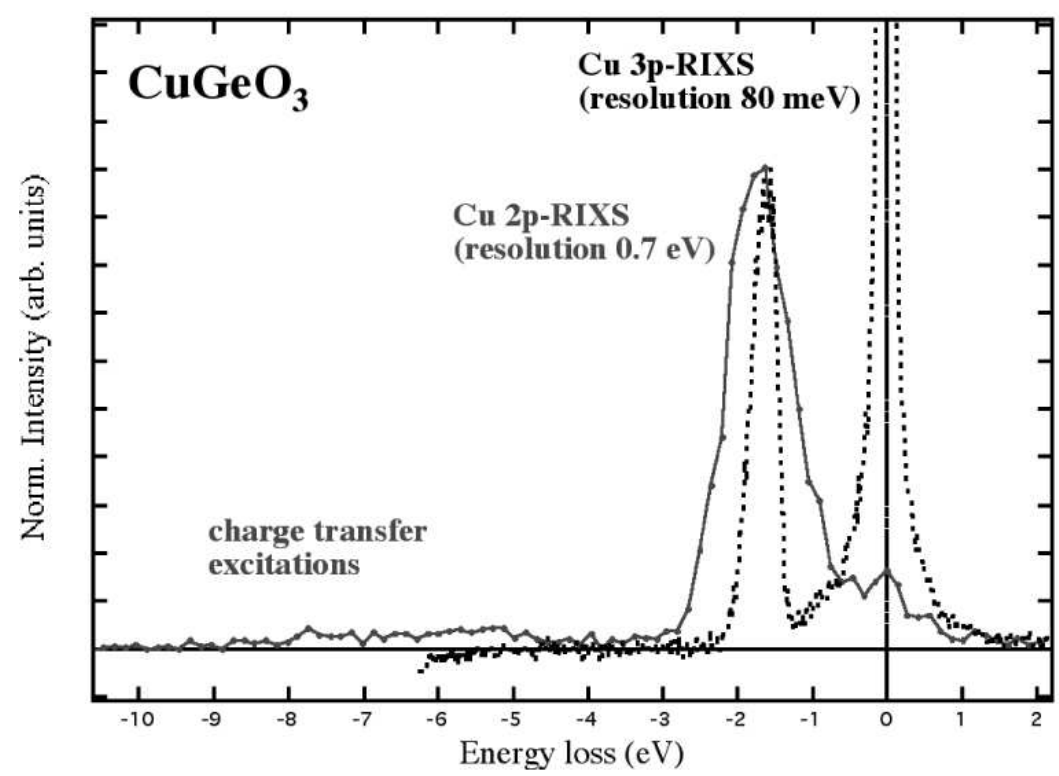

Figure 5.15: RIXS spectra of $\mathrm{CuGeO}_{3}$ at different core resonances: $\mathrm{Cu} 2 \mathrm{p}-\mathrm{RIXS}$ (solid line) and $\mathrm{Cu} 3 \mathrm{p}-\mathrm{RIXS}$ (dashed line). The peaks centered between $-1 \mathrm{eV}$ and $-2 \mathrm{eV}$ are due to dd-excitations. Charge transfer excitations are found at $-4 \mathrm{eV}$ to -8 $\mathrm{eV}$ in the $\mathrm{Cu} 2 \mathrm{p}$-RIXS spectra.

with energy values of dd-excitations found in infrared absorption spectroscopy. For $\mathrm{CuO}$ and $\mathrm{La}_{2} \mathrm{CuO}_{4}$ we observe two barely resolved structures, the energies of which are somewhat higher than previously published values using other spectroscopies. For $\mathrm{SrCuO}_{2}$, we observe three well-resolved excitations at $1.9 \mathrm{eV}, 2.2 \mathrm{eV}$, and $2.8 \mathrm{eV}$. These Raman shifts are extraordinarily large compared to the values of the other cuprates. Nevertheless an ab initio calculation that we present shows very good agreement to the experimentally determined values. We attribute this feature to the unique one-dimensional and compact structure of the cuprate $\mathrm{SrCuO}_{2}$. 


\section{Acknowledgments}

During the last years many people supported my work presented in this thesis in different ways and I would like to thank for the help I received from all of them (even if I might forget some of them) in the following.

First of all, I would like to express great gratitude to my main supervisor Prof. Joseph Nordgren, who gave me the opportunity to perform this work in his group.

Many thanks go to my supervisor Dr. Laurent Duda for introducing me into the field of soft X-ray emission spectroscopy and teaching me a great deal about the physics of transition metal oxides. Thanks for all the stimulating discussions, sharing many beamtime weeks together with me and all the help with preparing the manuscripts for this thesis.

It has been interesting to share the office with Magnus Ström, who also shares the same sense of working desk order as me. Thanks for all the help and nice company during these years.

Special thanks go to Andreas Augustsson, who never hesitated when help was needed, and for being a good friend. It was very nice that we had the opportunity to visit New York and Las Vegas together. Sorry that we never had the time to visit "Das Oktoberfest" in Munich:)

In particular, I would like to thank all present and former members of the group: Jan-Erik Rubensson, Jinghua Guo, Sergei Butorin, Tanel Käämbre, Marcus Agåker, Conny Såthe, Limin Qian, Emiliana Damian, Florentina Trif, Johan Söderström, Martin Magnuson, Johan Forsberg, Joachim Schiessling, Fouzia Salem, Kristina Kvashnina, Mikhail Yablonskikh, Minqi Wang, Pieter Kuiper and Lars Werme.

I gratefully acknowledge all recent Post-docs at beamline I511: Chris Glover, Lidia Gridneva, Tanel Käämbre, Lissy Kjeldgaard, and (nearly post-doc) Dennis Nordlund for the excellent job they did/do. Chungli Dong is thanked for his help at beamline 7.0.1 at ALS.

I am very grateful to Anne Kronquist for all the help with the paper work and all administrative support. Calle Englund is particularly acknowledged for always having a prefect solution for technical problems.

I would also like to thank all the people I had the pleasure to collaborate with during the last years. Especially, Maurizio Mattesini, Rajeev Ahuja, Dr. 
Masahiko Matsubara, Prof. Akio Kotani, Udo Schwingenschlögl, Volker Eyert, Matthias Klemm, Prof. Siegfried Horn, Patrice Millet, Sara Nordlinder, Jonas Höwing, Kristina Edström, Torbjörn Gustafsson, Prof. Kevin Smith, Cormac McGuinness, James Downes and D.D. Sarma.

I would like to thank my parents for their support and making it possible to study physics in the first place. Last, but not least many thanks to Florentina for her love and patience with me (and also for always helping me in Maxlab, when I get desperate:))

Thorsten Schmitt

Uppsala, April 26, 2004 


\title{
Summary in Swedish
}

\author{
Resonant Mjukröntgenspektroskopi av Vanadin- \\ oxider och Relaterade Föreningar
}

Ett material är sammansatt av atomer av samma eller olika slag. Dessa binds ihop av atomernas yttersta elektroner (valenselektroner) som fördelas på ett energi fördelaktigt sätt som kan beskrivas av kvantmekaniken. Egenskaperna hos materialet beror till största delen av hur elektronerna är fördelade mellan atomerna i materialet, d.v.s. materialets "elektronstruktur". I många övergångsmetalloxider är elektronerna av blandad karaktär beträffande lokalisering och delokalisering. Energinivåerna i lokaliserade material liknar atomära energitillstånd och har huvudsakligen bidrag i närheten av de specifika atomerna. Delokaliserade material bildar genom överlapp-ning av många atomära tillstånd ett gemensamt band av kvasi-kontinuerliga energier. Om växelverkan mellan olika elektroner inom materialet är relativt stor, kan man inte beskriva dem oberoende av varandra och man säger då att systemet är starkt korrelerat. Speciellt material med 4f och 3d elektroner som yttre valenselektroner anses vara starkt korrelerade. Korrelationens inverkan på elektronstrukturen leder till många intressanta fysikaliska materialegenskaper, som är mycket aktuella i dagens forskning.

Vanadinoxider tillhör en betydande klass av 3d övergångsmetalloxider, som har studerats mycket p.g.a. möjliga teknologiska applikationer. Olika vanadinoxider visar stora variationer i elektroniska, magnetiska och strukturella egenskaper och förståelsen av dessa fysiska egenskaper är direkt relaterat till de teknologiska tillämpningarna. Material baserade på vanadinoxider kan till exempel användas inom katalys och optoelektronik. Många vanadinoxider visar en övergång från metall till isolator eller halvledare vid en viss temperatur. Denna övergångstemperatur kan modifieras genom att substituera vanadinatomer med andra atomslag, s.k. dopning. Nyligen har det visats att vanadinbaserade material även är lovande som katoder för uppladdningsbara litiumbatterier, som idag används i t.ex. mobiltelefoner, digitalkameror samt bärbara datorer. $\mathrm{V}_{6} \mathrm{O}_{13}$, till exempel, möjliggör med sin öppna kristallstruktur, som bygger på $\mathrm{VO}_{6}$ enheter, en väldigt hög energitäthet samt god mobilitet av litiumjonerna under i- och ur-laddning.

Koppar-baserade material (i synnerhet kuprater) är intressanta bland annat för att de kan bli supraledande vid "relativt höga" temperaturer. Dessa kuprater 
med ett nästan fyllt 3d-skal visar stark korrelation. I denna avhandling studeras spektroskopiska egenskaper hos några isolerande kuprater vilka är modellsystem för förståelsen av supraledningsförmågan.

Elektronstrukturen hos olika material kan med fördel studeras med en rad olika spektroskopiska metoder. I denna avhandling har mjukröntgenspektroskopi används för att karakterisera elektronstrukturen i vanadin och kupratmaterial. Mjukröntgenspektroskopi skiljer sig från övriga spektroskopiska metoder genom att man bla. kan studera excitationer av inre elektroner en bit in i materialet. Mjukröntgen-absorption och -emission används för att undersöka besatta samt icke-besatta valenselektron-tillstånd. I mjukröntgenabsorption undersöks ickebesatta tillstånd genom att excitera en elektron från en inre nivå till lediga tillstånd i valensbandet med en röntgenfoton. Vid de-excitation kan samma eller en annan valenselektron fylla denna innerskalsvakans varvid en röntgenfoton emitteras under upprätthållandet av energibalansen. Denna foton registreras i mjukröntgenspektroskopi med en spektrometer, där fotonens energi eller våglängd bestäms. Spektrometern som används i detta arbete är en så kallad gitterspektrometer, som består av en ingångsspalt, tre sfäriska gitter (optimerade för olika energiområden) och en tvådimensionell detektor. Designprincipen bygger på Rowland-geometri, där samtliga ingående optiska element anordnas längs en cirkel. Gitterna är ofta tillverkade av glas belagt med ett tungt grundämne så som guld eller platina med många parallella ritsar (skåror).

I mjukröntgenområdet är en de-excitation där en foton sänds ut ca 100-1000 ggr svagare än den dominerande processen där en elektron sänds ut (en Augerelektron) för att upprätthålla energibalansen. Därför kräver denna spektroskopimetod avancerade ljusstarka excitationskällor. Röntgenstrålning av mycket hög intensitet kan fås med moderna s.k. synkrotronljuskällor där strålningen produceras genom att elektroner med relativistiska hastigheter förlorar energi i ett magnetfält. Alla experiment som är presenterade i denna avhandling är genomförda vid Nationella laboratorier vid MAX-lab (beläget vid Lund Universitet) i Lund samt vid ALS (Advanced Light Source) som ligger i Berkeley i närheten av San Francisco (Kalifornien) i USA.

Vid en synkrotronljusanläggning används även anordningar av optiska element, så som gitter, speglar och spalter, för att välja ut specifika fotonenergier samt för fokusering av strålningen till provet, som ska studeras. Genom att avstämma den inkommande strålningens energi till specifika resonanser i absorptionsspektret kan man få selektiv röntgenemission och därmed urskilja olika kemiska tillstånd för atomerna i ett material. För korrelerade material behöver man oftast beskriva absorptions-emission-processen som enstegs process, dvs. som resonant inelastisk röntgenspridning (förkortas RIXS på engelska). Genom dessa spridingsexperiment kan valensexcitationer studeras, så som laddningsöverföringar samt lokala övergångar mellan d-elektrontillstånd av olika symmetri.

Vanadinoxider med olika oxidationstal har studerats med mjukröntgenspektroskopi vid syre 1 s och vanadin 2 p-absorptionskanten för att karakterisera deras elektronstuktur. I samtliga material finns stark hybridisering mellan syre- och vanadinorbitalerna, som reflekteras i spektrumens utseende. Även lokala övergånger mel- 
lan vanadin 3d-tillstånd har observerats, som är relaterade till olika grad av lokalisering för dessa vanadin- material. För $\mathrm{VO}_{2}$ och $\mathrm{V}_{6} \mathrm{O}_{13}$ kunde effekten av fasövergången från metall till halvledare analyseras genom utvecklingen av olika band i vanadinspektret. Experimenten har jämförts med beräkningarna baserade på olika teortiska modeller. En klustermodell har används för att beskriva och simulera den observerade inelastiska röntgenspridningen. Bidrag från delokaliserade elektroner överenstämmer med beräkningar från en bandmodell. Elektronstrukturstudier av $\mathrm{Na}_{2} \mathrm{~V}_{3} \mathrm{O}_{7}$ nanotuber visar likheter med $\mathrm{V}_{6} \mathrm{O}_{13}$ och $\mathrm{NaV}_{2} \mathrm{O}_{5}$, som har en blandad valens med oxidationstal mellan fyra och fem. I litiumbatterier bestående av $\mathrm{V}_{6} \mathrm{O}_{13}$ och vanadin-nanotuber kunde laddningstransporten karakteriseras genom jämförelser med andra modellsystem. Olika isolerande kuprater har karakteriserats med mjukröntgenspridningen vid koppar 3p-kanten och syre 1skanten. Mycket bättre energiupplösning har uppnåtts med mätningar vid koppar 3p-kanten jämfört med tidigare uppmätta resultat vid koppar 2p-kanten. 



\section{Bibliography}

[1] P. A. Cox. Transition Metal Oxides - An Introduction to Their Electronic Structure and Properties. Oxford University Press, Walton Street, Oxford OX26DP, Oxford New York, 1995.

[2] G. Stefanovich, A. Pergament, and D. Stafanovich. J. Phys.: Condens. Matter 12, 8837 (2000).

[3] D.W. Murphy and P.A. Christian. Science 205, 651 (1979).

[4] T. Gustafsson, J. O. Thomas, R. Koksbang, and G. C. Farrington. Electrochimica Acta 37, 1639 (1992).

[5] D. Adler. Rev. Mod. Phys. 40, 714 (1968).

[6] M. Imada, A. Fujimori, and Y. Tokura. Rev. Mod. Phys. 70, 1039 (1998).

[7] G.A. Sawatzky. Experimental Studies of Electron Correlation Effects in Solids: in Electron Correlations and Materials Properties, edited by Gonis et al. Kluwer Academic/Plenum Publishers, 1999.

[8] E.J. Nordgren, S.M. Butorin, L.-C. Duda, J.-H. Guo, and J.-E. Rubensson. Soft $X$-ray Fuorescence Spectroscopy for Materials Science and Chemical Phyiscs: in Advanced Series in Physical Chemistry - Vol. 12A - Chemical Applications of Synchrotron Radiation. World Scientific, 2002. www.worldscientific.com.

[9] F. de Groot. Chem. Rev. 101, 1779 (2001).

[10] J.-E. Rubensson, J. Lüning, S. Eisebitt, and W. Eberhardt. Appl. Phys. A 65, 91 (1997).

[11] A. Kotani and S. Shin. Rev. Mod. Phys. 73, 203 (2001).

[12] S.M. Butorin. J. Electron Spectrosc. Relat. Phenom. 110-111, 213 (2000).

[13] J. Stöhr. NEXAFS Spectroscopy. Springer-Verlag, Berlin, Heidelberg, 1992.

[14] S. Hüfner. Photoelectron Spectroscopy. Springer-Verlag, Berlin Heidelberg, Germany, 1996. 
[15] K. Siegbahn, C. Nordling, G. Johansson, J. Hedman, P. F. Heden, K. Hamrin, U. Gelius, T. Bergmark, L. O. Werme, R. Manne, Y. Baer. ESCA-Applied to Free Molecules. North Holland, Amsterdam, 1969.

[16] M. O. Krause and J. H. Oliver. J. Phys. Chem. Ref. Data 8, 329 (1979).

[17] M. O. Krause. Phys. Rev. A 22, 1958 (1980).

[18] Faris Gel'mukhanov and Hans Ågren. Physics Reports 312, 87-330 (1999).

[19] J. J. Sakurai. Advanced Quantum Mechanics. Addison-Wesley, London, UK, 1967.

[20] H. A. Kramers and W. Heisenberg. Zeitschrift für Physik 31, 681 (1925).

[21] Hans Ågren and Faris Gel'mukhanov. J. Electron Spectrosc. Relat. Phenomena 110-111, 153-178 (2000).

[22] A. Kotani. J. Electron Spectrosc. Relat. Phenom. 110-111, 197 (2000).

[23] A. Kotani. Journal Phys. Chem. Sol. 61, 419 (2000).

[24] T. Ide and A. Kotani. J. Phys. Soc. Jpn. 67, 3621 (1998).

[25] A. Kotani and T. Ide. J. Synchrotron Radiat. 6, 308 (1999).

[26] Shriver and Atkins. Inorganic Chemistry. Oxford University Press, 1999. ISBN 0-521-65214-6.

[27] P. Hohenberg and W. Kohn. Phys. Rev. 136, B864 (1964).

[28] W. Kohn and L. J. Sham. Phys. Rev. 140, A1133 (1965).

[29] J. P. Perdew, K. Burke, and M. Ernzerhof. Phys. Rev. Lett., 77, 3865 (1996).

[30] J. Hubbard. Proc. Roy. Soc. London A 277, 237 (1964).

[31] P. W. Anderson. Phys. Rev. 124, 41 (1961).

[32] O. Gunnarsson and K. Schönhammer. Phys. Rev. B 28, 4315 (1983).

[33] M. Matsubara, T. Uozumi, A. Kotani, Y. Harada, and S. Shin. J. Phys. Soc. Jpn. 69, 1558 (2000).

[34] M. Matsubara, T. Uozumi, A. Kotani, Y. Harada, and S. Shin. J. Phys. Soc. Jpn. 71, 34 (2002).

[35] M. Matsubara, Y. Harada, S. Shin, T. Uozumi, and A. Kotani. J. Phys. Soc. Jpn. 73, 711 (2004).

[36] A. G. Michette and C. J. Buckley (Editors). X-ray Science and Technology. Institute of Physics Publishing, Bristol and Philadelphia, 1993. ISBN 0-75030233-X. 
[37] David Attwood. Soft X-rays and Extreme Ultraviolet Radiation. Cambridge University Press, 1999. ISBN 0-521-65214-6.

[38] J. D. Jackson. Classical Electrodynamics. John Wiley and Sons, New York, 1975 .

[39] J. Schwinger. Phys. Rev. 70, 798 (1946).

[40] J. Schwinger. Phys. Rev. 75, 1912 (1949).

[41] Tanel Käämbre. Resonant Soft X-Ray Spectroscopic Studies of $C_{60}$ and Related Materials. Acta Universitatis Upsaliensis, Uppsala, Sweden, 2002.

[42] W. B. Peatman. Gratings, Mirrors and Slits: Beamline Design for Soft XRay Synchrotron Radiation Sources. Gordon and Breach Science Publishers, Amsterdam, Netherlands, 1997.

[43] T. Warwick, P. Heimann, D. Mossessian, W. McKinney. Rev. Sci. Instrum. 66, 2037 (1995).

[44] R. Denecke, P. Väterlein, M. Bässler, N. Wassdahl, S. Butorin, A. Nilsson, J.-E. Rubensson, J. Nordgren, N. Mårtensson, and R. Nyholm. J. Electron Spectrosc. Relat. Phenom. 101-103, 971 (1999).

[45] Limin Qian. Soft X-Ray Spectroscopic Study of Fullerene Based TransitionMetal Compounds and Related Systems. Acta Universitatis Upsaliensis, Uppsala, Sweden, 2001.

[46] L. Kjeldgaard, C. Glover, L. Gridneva, and O. Karis. Max-lab beamline i511 lund university, sweden. [http://www.maxlab.lu.se/beamlines/bli511/], (2004).

[47] M. Agåker, A. Augustsson, T. Schmitt, T. Käämbre, L. Gridneva, K. Kvashnina, J.-E. Rubensson, and J. Nordgren. Measurements of beam size and optimising of refocusing optics on beamline i511. page, 200, 2000.

[48] J. Nordgren, G. Bray, S. Cramm, R. Nyholm, J.-E. Rubensson, and N. Wassdahl. Rev. Sci. Instr. 60, 1690 (1989).

[49] J. A. R. Samson. Techniques of Vacuum Ultraviolet Spectroscopy. Wiley, New York, 1967.

[50] Laurent Duda. Magnetic Dichroism and Polarized Soft X-Ray Spectroscopy of Transition Metal Compounds. Acta Universitatis Upsaliensis, Uppsala, Sweden, 1996.

[51] H. Schäfer. Chemische Transportreaktionen - Der Transport anorganischer Stoffe über die Gasphase und seine Anwendungen. Verlag Chemie GmbH, Weinheim/Bergstr., 1962.

[52] W. Brückner, H. Oppermann, W. Reichelt, J. I. Terukow, F. A. Tschudnowski, E. Wolf. Vanadiumoxide - Darstellung, Eigenschaften, Anwendungen. Akademie-Verlag, Berlin, 1983. 
[53] H. Paulin. Züchtung von Vanadiumoxideinkristallen und homologen einkristallinen Verbindungen mittels chemischem Transport. Diplomarbeit, Universität Augsburg, 1996.

[54] M. Isobe and Y. Ueda. J. Cryst. Growth 181, 314 (1997).

[55] P. Millet, J. Y. Henry, F. Mila, and J. Galy. J. Solid State Chem. 147, 676 (1999).

[56] A. Revcolevschi, U. Ammerahl, and G. Dhalenne. J. Cryst. Growth, 198-199, 593 (1999).

[57] C. Lampe-Önnerud and J. O. Thomas. Eur. J. Solid State Inorg. Chem. 32, 295 (1995).

[58] F. Krumeich, H.-J. Muhr, M. Niederberger, F. Bieri, B. Schnyder, and R. Nesper. J. Am. Chem. Soc. 121, 8324 (1999).

[59] S. Shin, S. Suga, M. Taniguchi, M. Fujisawa, H. Kanzaki, A. Fujimori, H. Daimon, Y. Ueda, K. Kosuge, and S. Kachi. Phys. Rev. B 41, 4993 (1990).

[60] T. Hrlin, T. Niklewski, and M. Nygren. Mat. Res. Bul. 8, 179 (1973).

[61] M. Nygren. Chem. Commun. 11, 1 (1973).

[62] R.M. Moon. Phys. Rev. Lett. 25, 527 (1970).

[63] P.D. Dernier and M. Marezio. Phys. Rev. B 2, 3771 (1970).

[64] C. Castellani, C.R. Natoli, and J. Ranninger. Phys. Rev. B 18, 5001 (1978).

[65] G.A. Thomas, D.H. Rapkine, S.A. Carter, A.J. Millis, T.F. Rosenbaum, P. Metcalf, and J.M. Honig. Phys. Rev. Lett. 73, 1529 (1978).

[66] P.B. Allen, R.M. Wentzcovitch, W.W. Schulz, and P.C. Canfield. Phys. Rev. $B$ 48, 4359 (1993).

[67] D.B. McWhan, M. Marezio, J.P. Remeika, and P.D. Dernier. Phys. Rev. B 10, 490 (1974).

[68] M. Gupta, A.J. Freeman, and D.E. Ellis. Phys. Rev. B 16, 3338 (1977).

[69] R.M. Wentzcovitch, J.L. Martins, and G.D. Price. Phys. Rev. Lett. 72, 3389 (1994).

[70] J.B. Goodenough. Phys. Rev. 117, 1442 (1960).

[71] C. Sommers, R. De Groot, D. Kaplan, and A. Zylbersztein. J. Phys. Lett. (Paris) 36, L157 (1975).

[72] E.Z. Kurmaev, V.M. Cherkashenko, Y.M. Yarmoshenko, S. Bartowski, A.V. Postnikov, M. Neumann, L.-C. Duda, J.-H. Guo, J. Nordgren, V.A. Perelyaev, and W. Reichelt. J. Phys.: Condens. Mater. 10, 4081 (1998). 
[73] V. Eyert, R. Horny, K.-H. Höck, and S. Horn. J. Phys.: Condens. Mater. 12, 4923 (2000).

[74] T. Christman, B. Felde, W. Niessner, D. Schalch, and A. Scharmann. Thin Solid Films 287, 134 (1996).

[75] M. Abbate, F.M.F. de Groot, J.C. Fuggle, Y.J. Ma, C.T. Chen, F. Sette, A. Fujimori, Y. Ueda, and K. Kosuge. Phys. Rev. B 43, 7263 (1991).

[76] H. Abe, M. Terauchi, M. Tanaka, S. Shin, and Y. Ueda. Jpn. J. Appl. Phys. 36, 165 (1997).

[77] O. Müller, E. Goering, J.P. Urbach, T. Weber, H. Paulin, M. Klemm, M.L. den Boer, and S. Horn. J. Phys. IV 7, C2-533 (1997).

[78] E. Goering, M. Schramme, O. Müller, R. Barth, H. Paulin, M. Klemm, M.L. den Boer, and S. Horn. Phys. Rev. B 55, 4225 (1997).

[79] E. Goering, M. Schramme, O. Müller, H. Paulin, M. Klemm, M.L. den Boer, and S. Horn. Physica B 230-232, 996 (1997).

[80] K.-A. Wilhelmi, K. Waltersson, and L. Kihlborg. Act. Chem. Scan. 25, 2675 (1971).

[81] P.D. Dernier. Mat. Res. Bull. 9, 955 (1974).

[82] N. Kimizuka, M. Nakano-Onoda, and K. Kato. Acta Cryst. B 34, 1037 (1978).

[83] R. Eguchi, T. Yokoya, T. Kiss, Y. Ueda, and S. Shin. Phys. Rev. B 65, 205124 (2002).

[84] H. Smolinski, C. Gros, W. Weber, U. Peuchert, G. Roth, M. Weiden, and C. Geibel. Phys. Rev. Lett. 80, 5164 (1998).

[85] M. Isobe and Y. Ueda. J. Phys. Soc. Jpn. 65, 1178 (1996).

[86] M. Lohmann, H.-A. Krug von Nidda, M. V. Eremin, A. Loidl, G. Obermeier, and S. Horn. Phys. Rev. Lett. 85, 1742 (2000).

[87] V.C. Long, Z. Zhu, J. L. Musfeldt, X. Wei, H.-J. Koo, M.-H. Whangbo, J. Jegoudez, and A. Revcolevschi. Phys. Rev. B 60, 15721 (1999).

[88] Z.S. Popovic and F.R. Vukajlovic. Phys. Rev. B 59, 5333 (1999).

[89] H. Wu and Q. Zheng. Phys. Rev. B 59, 15027 (1999).

[90] A.N. Yaresko, V.N. Antonov, H. Eschrig, P. Thalmeier, and P. Fulde. Phys. Rev. B 62, 15538 (2000).

[91] S. Atzkern, M. Knupfer, M. S. Golden, J. Fink, A. N. Yaresko, V. N. Antonov, A. Hübsch, C. Waidacher, K. W. Becker, W. von der Linden, G. Obermeier, and S. Horn. Phys. Rev. B 63, 165113 (2001). 
[92] G.P. Zhang, T.A. Callcott, G.T. Woods, L. Lin, B. Sales, D. Mandrus, and J. He. Phys. Rev. Lett. 88, 077401 (2002).

[93] S.A. Golubchik, M. Isobe, A.N. Ivlev, B.N. Mavrin, M.N. Popova, A.B. Sushkov, Y. Ueda, and A.N. Vasil'ev. J. Phys. Soc. Jpn. 66, 4042 (1997).

[94] M.J. Konstantinovic, J. Dong, M.E. Ziaei, B.P. Clayman, J.C. Irwin, K. Yakushi, M. Isobe, and Y. Ueda. Phys. Rev. B 63, 121102(R) (2001).

[95] J.-M. Tarascon and M. Armand. Nature 414, 359 (2001).

[96] Andreas Augustsson. RIXS Studies of Lithium Intercalation in Rechargeable Battery Systems. Licentiate Thesis, Uppsala University, Uppsala, Sweden, 2002.

[97] Jonas Höwing. The Challenge of Probing Lithium Insertion Mechanisms in Cathode Materials. Acta Universitatis Upsaliensis, Uppsala, Sweden, 2004.

[98] N.C. Chaklanabish and H.S. Maiti. Solid State Ionics 21, 207 (1986).

[99] P.E. Stallworth, S. Kostov, M.L. den Boer, S.G. Greenbaum, and C. LampeÖnnerud. J. Appl. Phys. 83, 1247 (1998).

[100] H. Bjö̈k, S. Lidin, T. Gustafsson, and J. O. Thomas. Acta Cryst. B 57, 759 (2001).

[101] Ö. Bergström, T. Gustafsson, and J. O. Thomas. Solid State Ionics 110, 179 (1998).

[102] Ö. Bergström, T. Gustafsson, and J. O. Thomas. Acta Cryst. C 54, 1204 (1998).

[103] S. Nordlinder, K. Edström, and T. Gustafsson. Electrochem. Solid State Lett. 4, A129 (2001).

[104] S. Nordlinder, J. Lindgren, T. Gustafsson, and K. Edström. J. Electrochem. Soc. 150, E280 (2003).

[105] S.M. Butorin, J.-H. Guo, N. Wassdahl, and J. Nordgren. J. Electron Spectrosc. Relat. Phenom. 110-111, 235 (2000).

[106] Janice L. Musfeldt. Spin-Peierls Materials, In: Magnetism: Molecules and Experiments, edited by J.S.Miller and M.Drillon. Wiley-VCH, 2000.

[107] C.T. Chen et al. Phys. Rev. Lett. 68, 2543 (1992).

[108] F.C. Zhang and T.M. Rice. Phys. Rev. B 37, 3759 (1988).

[109] H. Völlenke et al. Monatsh. Chem. 98, 1352 (1967).

[110] K. Okada and A. Kotani. Phys. Rev. B 63, 045103 (2001).

[111] K. Okada and A. Kotani. Phys. Rev. B 65, 144530 (2002).

[112] C. de Graaf and R. Broer. Phys. Rev. B 62, 702 (2000). 
[113] G. Ghiringhelli, N.B. Brookes, E. Annese, H. Berger, C. Dallera, M. Grioni, L. Perfetti, A. Tagliaferri, and L. Braicovich. Phys. Rev. Lett. 92, 117406 (2004). 


\section{Acta Universitatis Upsaliensis}

\section{Comprehensive Summaries of Uppsala Dissertations}

from the Faculty of Science and Technology

Editor: The Dean of the Faculty of Science and Technology

A doctoral dissertation from the Faculty of Science and Technology, Uppsala University, is usually a summary of a number of papers. A few copies of the complete dissertation are kept at major Swedish research libraries, while the summary alone is distributed internationally through the series Comprehensive Summaries of Uppsala Dissertations from the Faculty of Science and Technology. (Prior to October, 1993, the series was published under the title "Comprehensive Summaries of Uppsala Dissertations from the Faculty of Science".)

Distribution:

Uppsala University Library

Box 510, SE-751 20 Uppsala, Sweden

www.uu.se, acta@ub.uu.se 